To be cited as : Durand J-D, Hubert N, Shen K-N, Borsa P (2017) DNA barcoding grey mullets. Reviews in Fish Biology and Fisheries 27, 233-243. doi:10.1007/s11160-016-9457-7

\title{
DNA barcoding grey mullets
}

Jean-Dominique Durand $\cdot$ Nicolas Hubert $\cdot$ Kang-Ning Shen $\cdot$ Philippe Borsa

Jean-Dominique Durand

Institut de recherche pour le développement (IRD), MARBEC, Laboratory of Zoology, Department of Ecology and Evolutionary Biology, University of Science, VNUHCM 227 Nguyen Van Cu Str., Dist. 5 Ho Chi Minh City, Vietnam. E-mail: jeandominique.durand@ird.fr

Nicolas Hubert

IRD UMR 5554 ISEM, Jl. Raya Bogor Jakarta km 46, Cibinong 16911, Indonesia

Kang-Ning Shen

Department of Environmental Biology and Fisheries Science and Center of Excellence for Marine Bioenvironment and Biotechnology, National Taiwan Ocean University, Keelung 20224, Taiwan

Philippe Borsa

IRD UMR 250 “Ecologie marine tropicale des océans Pacifique et Indien”, Nouméa; e-mail: philippe.borsa@ird.fr 


\begin{abstract}
Despite the ecological and commercial importance of grey mullets (fish family Mugilidae), their taxonomy and systematics are still much debated. Reasons for this are the low level of morphometric variability and the relatively poor phylogenetic information borne by the morpho-anatomical characters used thus far in diagnosing species. Here, we evaluate the potential of DNA barcoding to accurately delineate species and assign unknown specimens to known taxa in the family Mugilidae. Our reference sample consists of 257 individuals from 91 lineages characterized by their nucleotide sequences at the COI, cytochrome $b$, and 16S rRNA loci. These lineages correspond to 55 species according to the current taxonomy, and 36 presumed cryptic species. All known and presumed cryptic species within the 'Mugil cephalus' $(n=15)$ and 'M. curema' $(n=6)$ species complexes, as well as within genera Chelon $(n=10)$, Crenimugil $(n=6)$, Osteomugil $(n=6)$, and Planiliza $(n=18)$ were successfully recovered as distinct lineages by $C O I$ gene sequences $(598 \mathrm{bp})$, demonstrating the utility of this marker to delineate species in the family Mugilidae. Inconsistencies in the labeling of sequences deposited in GenBank were ascribed to species misidentification. A proportion of these misidentifications occurred in the course of dedicated barcoding surveys, further emphasizing the need for an accurate and exhaustive reference barcoding database for Mugilidae.
\end{abstract}

Keywords Mugilidae $\cdot \mathrm{COI} \cdot$ Identification to species

\title{
Introduction
}

In the preamble to the eleventh volume of his and G. Cuvier's Histoire naturelle des poissons, Valenciennes (1836) confessed that their treatment of the Mugilidae had been a particularly laborious one: "La première famille dont nous traçons l'histoire dans ce volume est une de celles qui nous ont donné le plus de peine, à M. Cuvier et à moi". Leading taxonomists of the Mugilidae or grey mullets have emphasized the remarkably uniform appearance of fishes in this family, making straightforward diagnoses almost impossible for many species (Schultz 1946; Thomson 1966; Thomson 1997; Harrison and Senou 1999). As a possible consequence of these difficulties, grey mullets are often under-represented in field guides and other books on coastal fishes (e.g. Randall 1992; Shao et al. 1992; Allen 1997; Laboute and Grandperrin 2000; Randall 2005). Also, no morphology-based identification key is available for larvae and juveniles of the family Mugilidae $<60$ mm standard length (Thomson 1997; Leis and Carson-Ewart 2001) although some keys to Mugilidae species from particular areas have been proposed (van der Elst and Wallace 1976; Serventi et al. 1996).

Catalogues that identify commercial fishes from tissue samples using genetic markers or mass spectrophotometry techniques ignore a proportion of species in the Mugilidae, if not the family as a whole (e.g., Durand et al. 1985; Mazzeo et al. 2008). Ecological surveys and ichthyological inventories frequently fail to identify grey mullets to species (e.g. Chabanet and Durville 2005; Veiga et al. 2010; Franco et al.2012; Pogoreutz et al. 2012) or even to mention them (e.g., Mellin et al. 2016). Only 16 grey mullet species among a total of 51 listed by the Food and Agricultural Organization of the United Nations possess fishery statistics (http://www.fao.org/fishery/collection/asfis/en).

Yet, the accurate identification of grey mullets is fundamental to marine biodiversity research, as many species in this family are important from the point of view of the functional ecology of coastal ecosystems, including estuaries, mangroves, reefs, and lagoons (Blaber 1976; Blaber 2000; Lebreton et al. 2011; Chang and Iikuza 2012; Whitfield et al. 2012). Grey mullets are a valuable food resource and source of income for local communities in many tropical and temperate countries (Harrison and Senou 2002; Bernardon and Vall 2004;Guillemot et al. 2009; Jollit et al. 2010; Crosetti and Blaber 2016) and several species are of major importance to fisheries (Harrison and Senou 2002; Bacheler et al. 2005; Whitfield et al. 2012). Some mugilid species are also produced through intensive aquaculture (Harrison and Senou 2002; Gautier and Hussenot 2005; Whitfield et al.2012; Crosetti and Blaber 2016).

Molecular phylogenetics has demonstrated the occurrence of distinct and sometimes paraphyletic deep mitochondrial lineages in a proportion of the mugilid species, indicating a proportion of putative 
cryptic species (Shen et al. 2011; Durand et al. 2012b, McMahan et al.2013, Durand and Borsa 2015). As a consequence, the species richness of the family Mugilidae is thought to be currently underestimated and possibly largely so (Durand and Borsa 2015). DNA barcoding is expected to offer a shortcut to accurately identify grey mullets but also to delineate species and help flag presumed cryptic species. However, a first necessary step is to test whether the nucleotide sequence of the COI gene fragment used as the universal barcode in fishes (Ward et al. 2009) is powerful enough to distinguish both known species and presumed cryptic species in the Mugilidae. The aim of this paper is to assemble a comprehensive DNA-barcode library for grey mullets and to evaluate its effectiveness for identification to species.

\section{Materials and Methods}

\section{Nomenclature}

In this paper, genus nomenclature accords with the revisions of Durand et al. (2012a) and Xia et al. (2016). Nomenclature at the species level follows Durand and Borsa (2015).

Choice of a reference database

Durand et al.'s (2012a, b) trees based on concatenated partial $16 S r \mathrm{RN} A$, COI and cytochrome $b(c y t b)$ gene sequences ( $3,885 \mathrm{bp}$ long in total) of 257 reference specimens including 120 vouchers deposited in museum collections were used for the delineation of species and presumptive species (Durand and Borsa 2015). This is the reference database now used to assess the quality of DNAbarcoding in Mugilidae.

Genetic marker and evolutionary model

The marker here chosen as DNA barcode in Mugilidae was a 598-bp fragment of the COI gene comprised between nucleotide sites homologous to sites nos. 5624 and 6221 of the mitochondrial genome of Mugil sp. I (GenBank KM368340). The model of molecular evolution chosen for reconstructing the phylogeny of haplotypes was the Kimura 2-parameter (K2P) model (Kimura 1980). The algorithm used for constructing DNA-barcode trees was the Neighbor-Joining (NJ) algorithm (Saitou and Nei 1987), based on $\mathrm{K} 2 \mathrm{P}$ divergences. We are aware that the K2P model is not necessarily the most appropriate model to estimate nucleotide divergence between closely related COI gene sequences (Srivathsan and Meier 2012). Also, depending on the extent of sequence divergence and the length of the marker, the distance-based NJ algorithm may not always be as accurate as the character-based maximum-likelihood algorithm (Tateno et al. 1994). However, NJ trees of K2P distances have been the standard in barcoding surveys since the beginning of the COI barcoding initiative (Hebert et al. 2003). The first objective of the present work being to assess the utility and quality of standard COI barcodes in identifying grey mullets to species, standard data analysis had to be employed. Thus, for the purpose of the present work, we found no particular reason to switch to another model of molecular evolution and to another method of tree reconstruction.

Typology of genetic surveys of Mugilidae

Partial COI gene sequences deposited in GenBank and labeled as Mugilidae were downloaded on 21 February 2015. These were added to the COI gene sequence dataset of Durand et al. (2012a,b) with two 
objectives: (1) to eventually detect lineages that had not been sampled previously; (2) to eventually detect labelling errors of in the GenBank database.

Sequences from GenBank that did not include the entirety of a core segment 538-bp long, comprised between nucleotide sites nos. 5637 and 6173 in the mitochondrial genome of Mugil sp. I here used as a sequence of reference for the family (GenBank KM368340) were discarded. Sequences GenBank JF952795 and JF952796, labeled “Mugil cephalus" were also discarded because they clustered with the carangid Seriola sp. (see below). A total set of 829 partial COI-gene sequences, including the 261 sequences of Durand et al. (2012a, b) and 568 other sequences from GenBank, was used to produce an NJ tree of Mugilidae thus based on a segment 538-bp long.

Published genetic surveys of Mugilidae whose COI gene sequences were utilized in this paper (Supplementary Table S1) were categorized as "barcoding", "phylogeny" or "phylogeography" depending on their aims and content. For sequences from unpublished surveys, the category was inferred from the information provided in the GenBank record. "Barcoding" surveys were those aimed at providing COI gene sequences for the sole purpose of identifying species. "Phylogeny" surveys were concerned with the phylogeny or the molecular systematics at the supra-specific level. "Phylogeography" surveys were concerned with the genetic relationships among populations within a species or within closely related species of the same genus.

\section{Results}

Partial COI gene sequences as barcodes in grey mullets

The NJ tree of the COI barcodes of 54 individuals of the Mugil cephalus species complex (Fig. 1a) had the same basic topology as, and recovered all 15 lineages of Durand and Borsa's (2015) ML tree based on the concatenated partial COI, cytb and $16 S r R N A$ gene sequences (Supplementary Fig. S1a). This included $M$. cephalus, M. liza, and 13 other lineages denominated Mugil spp. A-L, Q (Durand and Borsa 2015). Bootstrap support to a lineage was $\geq 95 \%$ except for Mugil sp. H (72\%) and Mugil sp. I (60\%) (Fig. 1a).

The NJ tree of the COI barcodes of 38 individuals of the M. curema species complex (Fig. 1b) had almost the same topology as, and recovered all 6 lineages of Durand and Borsa's (2015) ML tree based on the concatenated partial COI, cytb and $16 \mathrm{~S} r \mathrm{RN} A$ gene sequences (Supplementary Fig. S1b). Bootstrap support to a lineage was $98 \%-99 \%$ (Fig. 1b).

The NJ tree of the COI barcodes of 52 individuals of the genera Crenimugil and Osteomugil (Fig. 2a) had almost the same topology as Durand et al.'s (2012b) ML tree based on the concatenated partial COI, cytb and $16 \mathrm{~S} r \mathrm{RN} A$ gene sequences (Supplementary Fig. S2a). All 12 lineages identified by Durand and Borsa (2015) were recovered in the COI tree. This included C. buchanani, C. crenilabis, three lineages under C. seheli designated as Crenimugil spp. A-C, an unidentified Crenimugil sp. sampled in Fiji and Taiwan (Crenimugil sp. D), three lineages under O. cunnesius referred to as Osteomugil spp. A-C, O. engeli, O. perusii, and O. robustus. Bootstrap support to a lineage was $97 \%-100 \%$ (Fig. 2a).

Likewise, all of the 28 lineages previously scored in the genera Chelon and Planiliza (Supplementary Fig. S2b) were recovered in the NJ tree of the COI barcodes (Fig. 2b), with bootstrap support to a lineage varying from $89 \%$ to $100 \%$.

Distribution of pairwise distances

The distribution of pairwise nucleotide distances based on the partial COI gene sequence dataset used for constructing the NJ tree of the M. cephalus species complex (Fig. 3a) showed three modal classes, at $0 \%$ - 
$0.5 \%, 1.5 \%-2.0 \%$, and $3.5 \%-4 \%$. Within-species (including presumed cryptic species) pairwise distances were all $<1 \%$, more than $90 \%$ of which were $<0.5 \%$. Inter-species distances ranged from $0.5 \%$ to $6.6 \%$. Thus, the first peak of the distribution corresponded mostly to intra-lineage distances whereas distances $>$ $1 \%$ exclusively concerned comparisons of haplotypes belonging to different lineages. The distribution of pairwise nucleotide distances in the M. curema species complex featured two non-overlapping modes peaking at $0 \%-0.5 \%$ (range: $0 \%-2.3 \%$ ) and at 5.5\%-6.0\% (range: $3.1 \%-9.2 \%$ ), respectively (Fig. 3b). The first mode exclusively consisted of within-lineage pairwise distances, whereas the second mode included all inter-lineage pairwise distances. In the genera Osteomugil and Crenimugil, pairwise nucleotide distances within a lineage were all $<3.5 \%$ whereas inter-lineage nucleotide distances were all $>3.5 \%$ (Fig. 3c). In the genera Chelon and Planiliza, pairwise nucleotide distances within a lineage were all $<3 \%$ whereas interlineage nucleotide distances were all $>4 \%$ (Fig. 3d).

New or undetermined mugilid lineages found by mining GenBank

The inclusion of all COI gene sequences available from GenBank (Supplementary Figs. S3-S9) uncovered 12 new mugilid lineages, i.e. lineages that were not present in our reference database (Durand et al. 2012a, b; Durand and Borsa 2015).

Haplotypes labeled “Agonostomus monticola” (GenBank JQ935845-JQ935849; Mejia et al. 2012) clustered as a new lineage sister to two other Dajaus spp. lineages (Dajaus monticola, Dajaus sp. B). These haplotypes likely belong to another yet undetermined species in the genus Dajaus (Supplementary Fig. S3).

A new Mugil sp. lineage was represented by three haplotypes from Saõ Paulo, Brazil (GenBank JQ365444-JQ365446; Ribeiro et al. 2012) and three from Belém, Brazil (GenBank JX185216-JX185218; Siccha-Ramirez et al. 2014); all six sequences were labeled "Mugil hospes". This lineage was sister to Mugil sp. R (Supplementary Fig. S3), of which it was separated by 7.5\% nucleotide divergence at the COI locus, and corresponds to the recently resurrected Mugil brevirostris (Menezes et al. 2015). Two new Mugil sp. lineages labeled "Mugil cephalus" in GenBank, represented each by 5 to 6 individuals sampled in Turkey (GenBank KC500933-KC500946; Keskin and Atar 2013) were sisters to M. cephalus (Supplementary Fig. S4) and were separated from it by 1.1-1.2\% nucleotide divergence at the COI locus. A haplotype originally labeled "Mugil curema" (GenBank DQ441603; Heras et al. 2006) was placed externally to the haplogroup representing the M. curema species complex as defined by Durand et al. (2012b) (Supplementary Fig. S3). This haplotype likely belongs to another yet undetermined or unsampled species in the genus Mugil. Also in the M. curema species complex (Supplementary Fig. S5), the recently-described M. margaritae (Menezes et al. 2015) corresponded to lineage Mugil sp. N of Durand and Borsa (2015). Last, two specimens labeled "Mugil cephalus" (GenBank JF952795, JF952796) placed at an odd position on a preliminary version of the NJ tree (not shown) turned out to be likely Seriola sp. as inferred from their 99\%-100\% homology with sequences of S. lalandi (GenBank EU752208) and S. quinqueradiata (GenBank EU752208, HQ641665HQ641667, KF202527).

Haplotypes originally labeled "Liza tade" (GenBank EU014262-EU01426 and JQ045776; Lakra et al. 2011, Ashiq Ur Rahman et al. 2013) were placed within the Osteomugil clade, as a new lineage distinct from all other known Osteomugil spp. lineages. This new lineage likely belongs to another yet undetermined or unsampled species in the genus Osteomugil (Supplementary Fig. S6). A haplotype labeled "Valamugil speiglern" (GenBank JQ045778; Ashiq Ur Rahman et al. 2013) came out as the sister lineage to O. cunnesius (Supplementary Fig. S6) and was separated from the latter by $4.1 \%$ nucleotide distance. This haplotype may effectively represent O. speigleri, which was absent from our reference database (Durand and Borsa 2015) or it may belong to another yet undetermined or unsampled species in the genus Osteomugil. A haplotype labeled "Sicamugil cascasia" (GenBank JX260972; Rathod et al. 2015), turned out to be placed 
within the Osteomugil clade as a lineage distinct from all known Osteomugil spp. lineages (Supplementary Fig. S6). With a nucleotide divergence of $13.8 \%$ from its closest relative, Osteomugil sp. B, this haplotype likely belongs to an undetermined or unsampled species in the genus Osteomugil.

No new lineage belonging to genera Crenimugil or Chelon was recovered (Supplementary Figs. S7, S8). The haplotype of an individual labeled"Chelon planiceps" from Vellar, Bay of Bengal (GenBank JQ045784) was placed outside Durand and Borsa's (2015) Planiliza sp. F and Planiliza sp. I [both formerly under P. tade, senior synonym of P. planiceps (Eschmeyer et al. 2016)] and all the other Planiliza spp. lineages sampled by Durand et al. (2012b) (Supplementary Fig. S9). This haplotype represents an undetermined lineage (Planiliza sp. of Supplementary Fig. S9) sister to P. subviridis. The haplotypes of two individuals labeled "Sicamugil cascasia" (GenBank JX983495, JX983496; Khedkar et al. 2014) were placed in the Planiliza clade, as a new lineage distinct from all other known Planiliza spp. lineages (Supplementary Fig. S9). This lineage represents another undetermined Planiliza sp. (Supplementary Fig. S9) closely related to P. subviridis and to the Planiliza sp. lineage represented by GenBank JQ045784 (see above). The haplotype of an individual from an undetermined species assigned to genus Liza (GenBank KC970393) formed a distinct lineage within Planiliza spp., which had not been previously sampled by us (Supplementary Fig. S9). Therefore, we assigned it to another undetermined Planiliza species. The haplotype of an individual labeled "Liza melinoptera" from the Philippines (GenBank KJ202170) formed a lineage sister to Durand and Borsa's (2015) Planiliza sp. G. This lineage likely represents a species distinct from Planiliza sp. G because of the substantial nucleotide divergence $(4.5 \%)$ with the latter.

Assessing the accuracy of published data

Among the 572 sequences retrieved from GenBank, 358 were linked to surveys assigned to the 'Barcoding" category, 90 were taken from 'Phylogeny' surveys, and 123 pertained to 'Phylogeography' surveys. Four hundred fifty two species labels matched our reference database, 84 were mismatches and the remainder could not be determined as matches or mismatches (Supplementary Table S1). Seventy six (90.5\%) mismatches were from 'Barcoding'-type surveys and the remaining eight (9.5\%) were from 'Phylogeny'type studies (Supplementary Table S1). Therefore, mismatches were much more frequent in 'Barcoding' studies than in 'Phylogeny' and 'Phylogeography' studies $\left(\chi^{2}\right.$ test; $\left.P<0.001\right)$. 


\section{Discussion}

In this study, we showed that a 598-bp long fragment of the COI gene is an adequete marker for identifying individuals to species in the family Mugilidae. This DNA barcode enabled us to detect misidentifications in previously published barcoding studies, This in turn enabled us to assign corrected species names to all mugilid COI gene sequences currently accessible in GenBank.

The COI gene fragment as barcode in Mugilidae

The Mugilidae has long been recognized as a difficult family regarding morphology-based taxonomy and species identification, due to the high level of morphological conservatism among species. We expected that the COI gene would provide a welcome alternative to morphological characters for identification to species in the Mugilidae. All currently recognized species and presumed cryptic species within genera Crenimugil, Osteomugil, Chelon and Planiliza and within the Mugil cephalus and the M. curema species complexes (Durand and Borsa 2015) were successfully recovered by using the sole COI-barcode. A barcoding gap was observed in five out of the six test groups used for the present survey, namely the M. curema speciescomplex and the genera Chelon, Crenimugil, Osteomugil and Planiliza. A barcoding gap was also visible in the M. cephalus species complex, except that a small percentage of intra-lineage pairwise genetic distances between haplotypes were overlapping with those between lineages.

The strength of the present results stems from the fact that a proportion of the species were sampled distribution-wide. For instance, several Indo-West Pacific species of the genera Planiliza, Osteomugil and Crenimugil were sampled from both the western Indian Ocean, the Coral Triangle (Taiwan, Philippines, West Papua) and the western Pacific. Both the M. cephalus and M. curema species complexes were sampled distribution-wide, with few geographic gaps. For these species complexes, barcoding gaps are unlikely to be artifacts resulting from loose geographic sampling. Thus, COI sequences provide molecular evidence to species delineation where traditional morphological characters may be poorly diagnostic if not mostly helpless, e.g. for distinguishing cryptic species within the $M$. cephalus species complex. The present results indicate that COI-barcoding is a powerful tool to identify species in, at least, the five most speciose genera of the family Mugilidae.

Inconsistencies in publicly available sequence databases

All COI gene sequences of Mugilidae presently available from GenBank were screened against our reference barcode database. A number of inconsistencies in the species labeling of sequences deposited in GenBank were observed. Further, a substantial proportion of the COI gene sequences downloaded from GenBank presented species labels different from our reference sequence database. A mismatch may either indicate mitochondrial introgression of an individual correctly identified to species, or result from misidentification, or be caused by the inadvertent inversion of samples at some stage in the experiments or by contamination. In a number of cases $(N=28)$, the mitochondrial lineage belonged to a genus different from the one assigned to the sequence in GenBank (e.g. P. affinis for GenBank's "Valamugil engell'). In the other cases $(N=52)$, the sequence apparently belonged to a distantly related species within the same genus (e.g. M. trichodon for GenBank's "Mugil cephalus"; P. affinis for GenBank's "Liza subviridis"). Therefore, we deemed the introgression hypothesis unlikely, because the mismatches concerned either species from different genera, or phylogenetically distant species within a genus, but not phylogenetically close species within a genus. We are then tempted to consider that most if not all mismatches in the published dataset that we examined likely correspond to misidentifications. Another possibility is 
contaminations from other samples at one of the different steps of the barcoding process. This would explain the confusion between sequences from different fish families.

DNA barcoding studies are supposed to provide reference nucleotide-sequence databases to be used in subsequent ecological, fisheries, food and other types of studies that use DNA barcodes to identify species. The likely misidentifications of mugilid species in GenBank were more frequent in barcoding studies than in the two other types of studies that require the use of genetic markers (namely, phylogeny and phylogeography) and where accurate identification to species is also fundamental. Failure of some barcoding studies to provide accurate species identification possibly relate to the difficulty to gather a reliable systematic expertise for a wide range of fish taxa, which is the case when the DNA barcoding approach is done from a bulk sampling that did not target specific families, as do phylogeny surveys, or species, as do phylogeographic surveys. The present survey thus implicitly confirms that species in the family Mugilidae are particularly difficult to identify using morphological characters, and all the more emphasizes the utility of sound DNA barcoding in this family.

Potential use of the COI gene fragment for the taxonomic revision of Mugilidae

DNA-barcoding also proved a valuable tool to extend the Linnean approach to taxonomy in grey mullets. Overall, 97 deeply rooted lineages were observed in the present study, which we previously assumed to be likely distinct species (Durand and Borsa 2015; present study). A small majority (53) of these species have a valid name, while the remaining 44 have yet to be either resurrected or described anew. Therefore, considerable effort is still required to clarify the taxonomy of species in the family Mugilidae. Because it can now be assumed with a high degree of confidence that COI barcodes are diagnostic to most if not all mugilid species, they should become useful, and even essential to their delineation.

Acknowledgments A preliminary version of this paper was presented the Fish Barcode of Life World Conference in Yeosu (South Korea) in June 2012, whose organizing committee JDD is grateful to. Pre-twentieth century books and articles were consulted online from the Biodiversity Heritage Library website (http://www.biodiversitylibrary.org/).

Authors' contributions PB, JDD, NH and KNS designed the project; PB and JDD participated in the collection, identification and curation of samples; JDD and KNS supervised the laboratory work; PB and JDD analyzed and interpreted the data, and wrote the manuscript; NH edited a pre-final version; all authors read and approved the final manuscript.

Data accessibility The sequences are available from Genbank (see supplementary Table S1 for accession numbers). Supplementary materials are available from the Hal-IRD open-access online repository (http://www.hal.ird.fr/; hal@ird.fr). 


\section{References}

Allen G (1997) Marine fishes of tropical Australia and Southeast Asia. Western Australian Museum, Perth, $292 \mathrm{p}$

Ashiq Ur Rahman M, Ajmal Khan S, Lyla PS, Prasanna Kumar C (2013) DNA barcoding resolves taxonomic ambiguity in

Mugilidae of Parangipettai waters (southeast coast of India). Turk J Fish Aquat Sci 13:321-330

Bacheler NM, Wong RA, Buckel JA (2005) Movements and mortality rates of striped mullet in North Carolina. N Am J Fish Manage 25:361-373

Bernardon M, Vall MOM (2004) Le mulet en Mauritanie: biologie, écologie, pêche et aménagement. Fondation internationale du Banc d'Arguin, Morges, $53 \mathrm{p}$

Blaber SJM (1976) The food and feeding ecology of Mugilidae in the St Lucia lake system. Bio J Linn Soc 8:267-277

Blaber SJM (2000) Tropical estuarine fishes: ecology, exploitation and conservation. Blackwell Science, Oxford, $372 \mathrm{p}$

Chabanet P, Durville P (2005) Reef fish inventory of Juan de Nova's natural park (western Indian Ocean). WIO J Mar Sci 4:145162

Chang C-W, IizukaY (2012) Estuarine use and movement patterns of seven sympatric Mugilidae fishes: the Tatu Creek estuary, central western Taiwan. Estuar Coast Shelf Sci 106:121-126

Crosetti D, Blaber SJM (2016) Biology, ecology and culture of grey mullets (Mugilidae). CRC Press, Boca Raton FL, 539 p

Durand J-D, Borsa P (2015) Mitochondrial phylogeny of grey mullets (Acanthopterygii: Mugilidae) suggests high proportion of cryptic species. C R Biol 338:266-277

Durand J-D, Chen W-J, Shen K-N, Fu C, Borsa P (2012a) Genus-level taxonomic changes implied by the mitochondrial phylogeny of grey mullets (Teleostei: Mugilidae). C R Biol 335:687-697

Durand J-D, Shen K-N, Chen W-J, Jamandre B-W, Blel H, Diop K, Nirchio M, Garcia de Leon FJ, Whitfield AK, Chang C-W, Borsa P (2012b) Systematics of the grey mullets (Teleostei: Mugiliformes: Mugilidae): molecular phylogenetic evidence challenges two centuries of morphology-based taxonomy. Mol Phyl Evol 64:73-92

Durand P, Landrein A, Quéro J-C (1985) Catalogue électrophorétique des poissons commerciaux. IFREMER, Nantes, $198 \mathrm{p}$ Eschmeyer WN, Fricke R, van der Laan R (eds) (2016) Catalog of fishes: genera, species, references.California Academy of Sciences, San Francisco (http://researcharchive.calacademy.org/research/ichthyology/catalog/fishcatmain.asp). Electronic version accessed July 2016

Franco A, Pérez-Ruzafa A, Drouineau H, Franzoi P, Koutrakis ET, Lepage M, Verdiell-Cubedo D, Bouchoucha M, López-Capel A, Riccato F, Sapounidis A, Marcos C, Oliva-Paterna FJ, Torralva-Forero M, Torricelli P (2012) Assessment of fish assemblages in coastal lagoon habitats: effect of sampling method. Estuar Coast Shelf Sci 112:115-125

Gautier D, Hussenot J (2005) Les mulets des mers d'Europe. Synthèse des connaissances sur les bases biologiques et les techniques d'aquaculture. Ifremer, Plouzané, $120 \mathrm{p}$

Guillemot N, Léopold M, Cuif M, Chabanet P (2009) Characterization and management of informal fisheries confronted with socio-economic changes in New Caledonia (South Pacific). Fish Res 98:51-61

Harrison IJ, Senou H (1999) Mugilidae. In: Carpenter KE, Niem VH (eds), FAO species identification guide for fishery purposes. The living marine resources of the Western Central Pacific, vol. 4: Bony fishes part 2 (Mugilidae to Carangidae). FAO, Rome, p 2069-2108

Hebert PD, Cywinska A, Ball SL, de Waard JR (2003) Biological identifications through DNA barcodes. Proc R Soc B 270:313321

Heras S, Gonzalez Castro M, Roldan MI (2006) Mugil curema in Argentinean waters: combined morphological and molecular approach. Aquaculture261:473-478

Jollit I, Léopold M, Guillemot N, David G, Chabanet P, Lebigre J-M, Ferraris J (2010) Geographical aspects of informal reef fishery systems in New Caledonia.Mar Pollut Bull 61:585-597

KeskinE,Atar HH (2013) DNA barcodingcommercially important fish species of Turkey. MolEcol Res 13:788-797

Khedkar GD, Jamdade R, Naik S, David L, Haymer D (2014) DNA barcodes for the fishes of the Narmada, one of India's longest rivers. PLoS One 9:e10146 
Kimura M (1980) A simple method for estimating evolutionary rate of base substitutions through comparative studies of nucleotide sequences. J Mol Evol 16:111-120

Laboute P, Grandperrin R (2000) Poissons de Nouvelle-Calédonie. Editions Catherine Ledru, Nouméa, 520 p

Lakra WS, Verma MS, Goswami M, Lal KK, Mohindra V, Punia P, Gopalakrishnan A, Singh KV, Ward RD, Hebert P (2011) DNA barcoding Indian marine fishes. Mol Ecol Res 11:60-71

Lebreton B, Richard P, Parlier EP, Guillou G, Blanchard GF (2011) Trophic ecology of mullets during their spring migration in a European saltmarsh: a stable isotope study. Estuar Coast Shelf Sci 91:502-510

Leis JM, Carson-Ewart BM (2001) The larvae of Indo-Pacific coastal fishes. An identification guide to marine fish larvae. Brill, Leiden, $850 \mathrm{p}$

Mazzeo MF, DeGiulio B, Guerriero G, Ciarcia G, Malorni A, Russo GL, Siciliano RA (2008) Fish authentication by MALDITOF mass spectrometry. J Agric Food Chem 56:11071-11076

McMahan CD, Davis MP, Dominguez-Dominguez O, Garcia-de- Leon FJ, Doadrio I, Piller KR (2013) From the mountains to the sea: phylogeography and cryptic diversity within the mountain mullet, Agonostomus monticola (Teleostei: Mugilidae). J Biogeogr 40:894-904

Mejia O, Leon-Romero Y, Soto-Galera E (2012) DNA barcoding of the ichthyofauna of Panuco-Tamesi complex: evidence for taxonomic conflicts in some groups. Mitochondr DNA 23:471-476

Mellin C, Mouillot D, Kulbicki M, McClanahan TR, Vigliola L, Bradshaw CJA, Brainard RE, Chabanet P, Edgar GJ, Fordham DA, Friedlander AM, Parravicini V, Sequeira AMM, Stuart-Smith RD, Wantiez L,Caley MJ (2016) Humans and seasonal climate variability threaten large-bodied coral reef fish with small ranges. Nature Comm 7:10491

Menezes NA, Nirchio M, De Oliveira C, Siccha-Ramirez R (2015) Taxonomic review of the species of Mugil (Teleostei: Perciformes: Mugilidae) from the Atlantic South Caribbean and South America, with integration of morphological, cytogenetic and molecular data. Zootaxa 3918:1-38

Pogoreutz C, Kneer D, Litaay M, Asmus H, Ahnelt H (2012) The influence of canopy structure and tidal level on fish assemblages in tropical Southeast Asian seagrass meadows. Estuar Coast Shelf Sci 107: 58-68

Randall JE (1992) Diver's guide to fishes of the Maldives. Immell, London, $193 \mathrm{p}$

Randall JE (2005) Reef and shore fishes of the South Pacific. New Caledonia to Tahiti and the Pitcairn Islands. University of Hawai'i Press, Honolulu, xii $+707 \mathrm{p}$

Rathod S, Kalyankar A, Tiknaik A, Jamdade R, Khdkar G (2015) DNA barcodes for the fish of the second-largest river of India: the Godavari. Genome 58:269-269

Ribeiro AO, Caires RA, Mariguela TC, Pereira LH, Hanner R, Oliveira C (2012) DNA barcodes identify marine fishes of Sao Paulo State, Brazil. Mol Ecol Resour 12:1012-1020

Saitou N, Nei M (1987) The neighbor-joining method: a new method for reconstructing phylogenetic trees. Mol Biol Evol 4:406425

Schultz LP (1946) A revision of the genera of mullets, fishes of the family Mugilidae, with descriptions of three new genera. Proc U S Natl Mus 96:377-395

Serventi M, Harrison IJ, Torricelli P, Gandolfi G (1996) The use of pigmentation and morphological characters to identify Italian mullet fry. J Fish Biol 49:1163-1173

Shao K-T, Chen J-P, Shen S-C (1992) Marine fishes of the Ken-Ting national park. Ministry of Interior Marine National Park Headquarters, Kaohsiung, $427 \mathrm{p}$

Shen K-N, Jamandre BW, Hsu C-C, Tzeng W-N, Durand J-D (2011) Plio-Pleistocene sea level and temperature fluctuations in the northwestern Pacific promoted speciation in the globally-distributed flathead mullet Mugil cephalus. BMC Evol Biol $11: 83$

Siccha-Ramirez R, Menezes NA,Nirchio M, Foresti F, Oliveira C (2014) Molecular identification of mullet species of the Atlantic South Caribbean and South America and the phylogeographic analysis of Mugil liza. Rev Fish Biol Fish 22:86-96

Srivathsan A, Meier R (2012) On the inappropriate use of Kimura-2-parameter (K2P) divergences in the DNA-barcoding literature. Cladistics 28:190-194 
Tamura K, Peterson D, Peterson N, Stecher G, Nei M, Kumar S (2011) MegA5: Molecular evolutionary genetics analysis using maximum likelihood, evolutionary distance, and maximum parsimony methods. Mol Biol Evol 28:2731-2739

Tateno Y, Takezaki N, Nei M (1994) Relative efficiencies of the Maximum-Likelihood, Neighbor-Joining, and MaximumParsimony methods when substitution rate varies with site. Mol Biol Evol 11:261-277

Thomson JM (1966) The grey mullets. Oceanogr Mar Biol 4:301-355

Thomson JM (1997) The Mugilidae of the world. Mem Queensl Mus 43:457-562

Valenciennes A (1836) Livre XIII: Mugilö̈des. In Cuvier G, Valenciennes A (eds) Histoire naturelle des poissons, tome onzième. F.G. Levrault, Paris, p 1-186, pls 307-318

van der Elst RP, Wallace JH (1976) Identification of the juvenile mullet of the east coast of South Africa. J Fish Biol 9:371-374

Veiga P, Ribeiro J, Gonçalves JMS, Erzini K (2010) Quantifying recreational shore angling catch and harvest in southern Portugal (north-east Atlantic Ocean): implications for conservation and integrated fisheries management. J Fish Biol76: 2216-2237

Ward RD, Hanner R, Hebert PDN (2009) The campaign to DNA barcode all fishes, FISH-BOL. J Fish Biol 74:329-356

Whitfield AK, Panfili J, Durand J-D (2012) A global review of the cosmopolitan flathead mullet Mugil cephalus Linnaeus 1758 (Teleostei: Mugilidae), with emphasis on the biology, genetics, ecology and fisheries aspects of this apparent species complex. Rev Fish Biol Fish 22:641-681

Xia R, Durand J-D, Fu C (2016) Multilocus resolution of Mugilidae phylogeny (Teleostei: Mugiliformes): Implications for the family'staxonomy. Mol Phyl Evol 96:161-177 


\section{Legends to figures}

Fig. 1 Neighbor-Joining trees constructed from Kimura 2-parameter distances (MEgA5: Tamura et al. 2011) for a 598-bp fragment of the COI gene. Each individual is designated by its number as in Durand and Borsa (2015) and by its geographic origin. Vertical grey bars on the right of the tree indicate COI haplotype clusters; vertical black bars indicate the lineages previously uncovered by the mitochondrial phylogeny of Durand et al. (2012a, b). Bootstrap score indicated at a node when > 50\%. a Mugil cephalus species complex, including M. cephalus, M. liza, and Mugil spp. A-L, Q of Durand and Borsa (2015). b Mugil curema species complex, including M. curema, M. incilis, M. thoburni, and Mugil spp. M-O of Durand and Borsa (2015)

Fig. 2 Neighbor-Joining trees constructed from Kimura 2-parameter distances (MEgA5: Tamura et al. 2011) for a 598-bp fragment of the COI gene. Each individual is designated by its number as in Durand and Borsa (2015) and by its geographic origin. Vertical grey bars on the right of the tree indicate COI haplotype clusters; vertical black bars indicate the lineages previously uncovered by the mitochondrial phylogeny of Durand et al. (2012b). Bootstrap score indicated at a node when $>50 \%$. a Species in the genus Crenimugil including C. buchanani, C. crenilabis, and Crenimugil spp. A-D of Durand and Borsa (2015) and in the genus Osteomugil, including O. cunnesius, O. engeli, O. perusii, O. robustus, and Osteomugil A, B of Durand and Borsa (2015). b Species in the genus Chelon including C. auratus, C. bandialensis, C. dumerili, C. labrosus, C. ramado, C. richardsonii, C. saliens, C. tricuspidens, and Chelon spp. A-B of Durand and Borsa (2015) and in the genus Planiliza, including P. abu, P. affinis, P. alata, P. baematocheila, P. macrolepis, P. melinoptera, P. ordensis, P. subviridis, and Planiliza A-J of Durand and Borsa (2015)

Fig. 3 Distribution of pairwise Kimura 2-parameter distances (MEgA5: Tamura et al. 2011) between haplotypes, estimated from the nucleotide sequences of a 598-bp fragment of the COI gene. Solid bars: within-lineage (i.e., within species or presumed species) distances; open bars: distances between lineages. a Mugil cephalus species complex, based on the sequences of Fig. 1a. b M. curema species complex, based on the sequences of Fig. 1b. c Crenimugil spp. and Osteomugil spp., based on the sequences of Fig. 2a. d Chelon spp. and Planiliza spp., based on the sequences of Fig. $2 \mathrm{~b}$ 


\section{Supplementary Material}

Supplementary Table S1. List of individual sequences at the COI locus, with sampling details, individual numbers, voucher details and GenBank accession numbers (http://www.ncbi.nlm.nih.gov/; accessed 21 Feb. 2015), including all reference individuals from a previous phylogenetic survey (Durand et al. 2012a, b; Durand and Borsa 2015). Label (GenBank) original species label in GenBank; Species identification identification from COI barcode (Figs. S3-S9) against Durand and Borsa's (2015) reference dataset [for all sequences retrieved from GenBank except those deposited by Durand et al.(2012a, b)]. Assessment of the accuracy of identification of grey mullet specimens used in barcoding, phylogenetic, and phylogeographic surveys: $M$ match; $X$ mismatch; $u$ undetermined.

Supplementary Fig. S1 Phylogenetic trees of mitochondrial haplotypes constructed from partitioned maximum-likelihood analysis of 3,885 aligned nucleotides from 16S rRNA, COI and $y t b$ gene sequences (edited from Durand et al. 2012b). Bootstrap scores $>50 \%$ are indicated. Asterisks indicate nodes with a posteriori probability from partitioned Bayesian analysis $>0.95$. Vertical bars on the right of the tree indicate statistically supported lineages that have been considered to represent distinct species (Durand and Borsa 2015) a Mugil cephalus species complex. b Mugil curema species complex

Supplementary Fig. S2 Phylogenetic trees of mitochondrial haplotypes constructed from partitioned maximum-likelihood analysis of 3,885 aligned nucleotides from 16S rRNA, COI and $y t b$ gene sequences (edited from Durand et al. 2012b). Bootstrap scores $>50 \%$ are indicated. Asterisks indicate nodes with a posteriori probability from partitioned Bayesian analysis $>0.95$. $V$ ertical bars on the right of the tree indicate statistically supported lineages that have been considered to represent distinct species (Durand and Borsa 2015). a Genera Osteomugil and Crenimugil, as redefined by Durand et al. (2012a). b Genera Chelon and Planiliza, as redefined by Durand et al. (2012a)

Supplementary Fig. S3 Neighbor-joining tree of 829 partial COI-gene sequences belonging to Mugilidae, including the 261 sequences of Durand et al. (2012 a,b) used as reference and 568 other sequences retrieved from GenBank on 21 February 2015. Distances were estimated using the K2P model in MEGA5 (Tamura et al. 2011). Bootstrap score indicated at a node when $>70 \%$. Vertical bars on the right of the tree indicate strongly-supported lineages that represent distinct species according to Durand and Borsa (2015). Sub-trees of the Mugil cephalus and the M. curema species complexes are shown in Supplementary Figs. 4 and 5 , respectively; those for the genera Osteomugil, Crenimugil, Chelon, and Planiliza are shown in Supplementary Figs. 6-9, respectively

Supplementary Fig. S4 Detail of sub-tree that represents COI haplotypes in the Mugil cephalus species complex, from the NJ tree of Supplementary Fig. S3. Bootstrap score indicated at a node when $>70 \%$. Vertical bars on the right of the tree indicate strongly-supported lineages that represent distinct species according to Durand and Borsa (2015)

Supplementary Fig. S5 Detail of sub-tree that represents COI haplotypes in the Mugil curema species complex, from the NJ tree of Supplementary Fig. S3. Bootstrap score indicated at a node when $>70 \%$. Vertical bars on the right of the tree indicate stronglysupported lineages that represent distinct species according to Durand and Borsa (2015)

Supplementary Fig. S6 Detail of sub-tree that represents COI haplotypes in the genus Osteomugil, from the NJ tree of Supplementary Fig. S3. Bootstrap score indicated at a node when $>70 \%$. Vertical bars on the right of the tree indicate stronglysupported lineages that represent distinct species according to Durand and Borsa (2015)

Supplementary Fig. S7 Detail of sub-tree that represents COI haplotypes in the genus Crenimugil, from the NJ tree of Supplementary Fig. S3. Bootstrap score indicated at a node when $>70 \%$. Vertical bars on the right of the tree indicate stronglysupported lineages that represent distinct species according to Durand and Borsa (2015) 
Supplementary Fig. S8 Detail of sub-tree that represents COI haplotypes in the genus Chelon, from the NJ tree of Supplementary Fig. S3. Bootstrap scores are indicated at a node when $>70 \%$. Vertical bars on the right of the tree indicate strongly-supported lineages that represent distinct species according to Durand and Borsa (2015)

Supplementary Fig. S9 Detail of sub-tree that represents COI haplotypes in the genus Planiliza, from the NJ tree of Supplementary Fig. S3. Bootstrap scores indicated at a node when $>70 \%$. Vertical bars on the right of the tree indicate stronglysupported lineages that that represent distinct species according to Durand and Borsa (2015) 
a

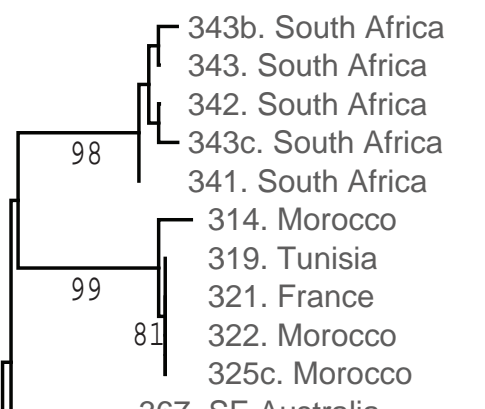

72- 367. SE Australia 362b. New Zealand 377. SE Australia

75 358. China

$60-328$. Taiwan

90 357. Taiwan

75 362. New Zealand

388. New Caledonia

95 361. New Zealand

\begin{tabular}{l|l} 
350. Hawaii \\
351. Hawaii \\
354b. Hawaii
\end{tabular} 375. Chile
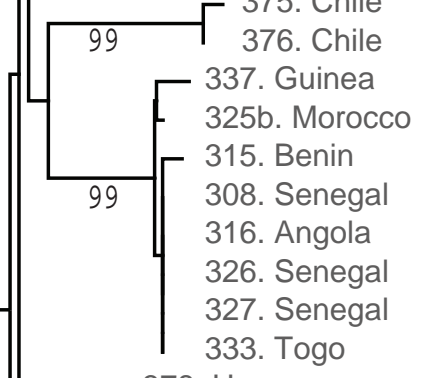

378. Uruguay

98 379. Uruguay 295. F. Guyana 298. Venezuela 329. Taiwan 368. W. Australia 6446. Galapagos

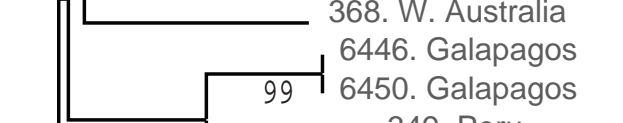
349. Peru

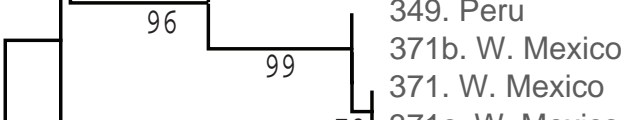

72 371c. W. Mexico
373. E. Mexico L 344. E. U.S.A.

99 347. E. U.S.A.

71 380. New Caledonia 381. Fiji

383. New Caledonia 329b. Taiwan 384. New Caledonia 385. New Caledonia 386. New Caledonia 0.01 387. New Caledonia

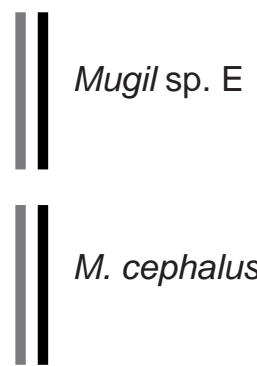

Mugil sp. H

Mugil sp. I

Mugil sp. F

Mugil sp. D

Mugil sp. A

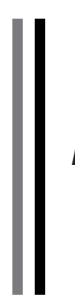

Mugil sp. J

M. liza

I Mugil sp. C

I Mugil sp. K

I Mugil sp. Q

Mugil sp. G

Mugil sp. B b

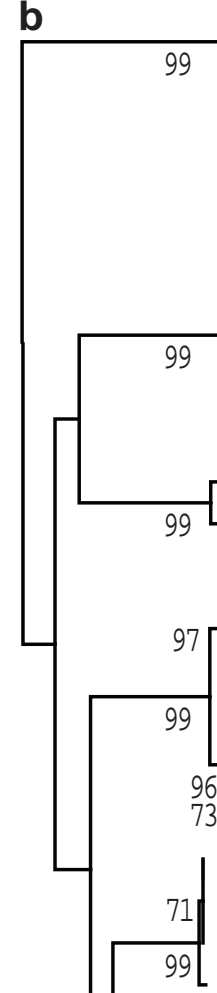

299. Fr. Guyana

302. Fr. Guyana

M. incilis

407. E. Mexico

404. E. U.S.A.

417. Uruguay

-405. Honduras

411. Guadeloupe

419. Belize

408. Brazil

414. Venezuela

418. Uruguay

6435. Galapagos

6445. Galapagos

M. thoburni

392. Senegal

391. Senegal

394. Benin

397. Togo

390. Senegal

396. Togo

73 393. Benin

401. Venezuela

399. E. Panama

403. Venezuela

99 400. E. Panama

Mugil sp. N

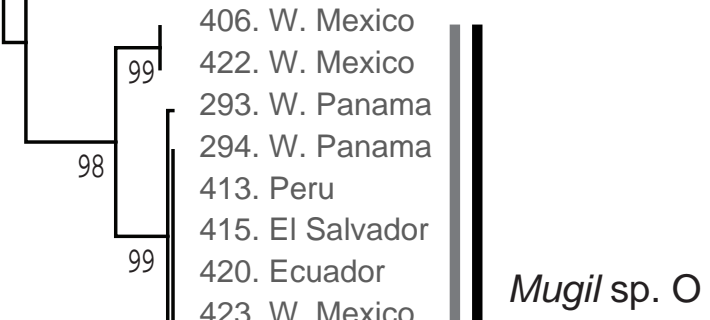

423. W. Mexico

424. W. Mexico

425. W. Mexico

426. El Salvador

429. El Salvador

430. El Salvador

432. El Salvador

M. curema

Mugil sp. M

Mugil sp. O

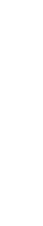

列



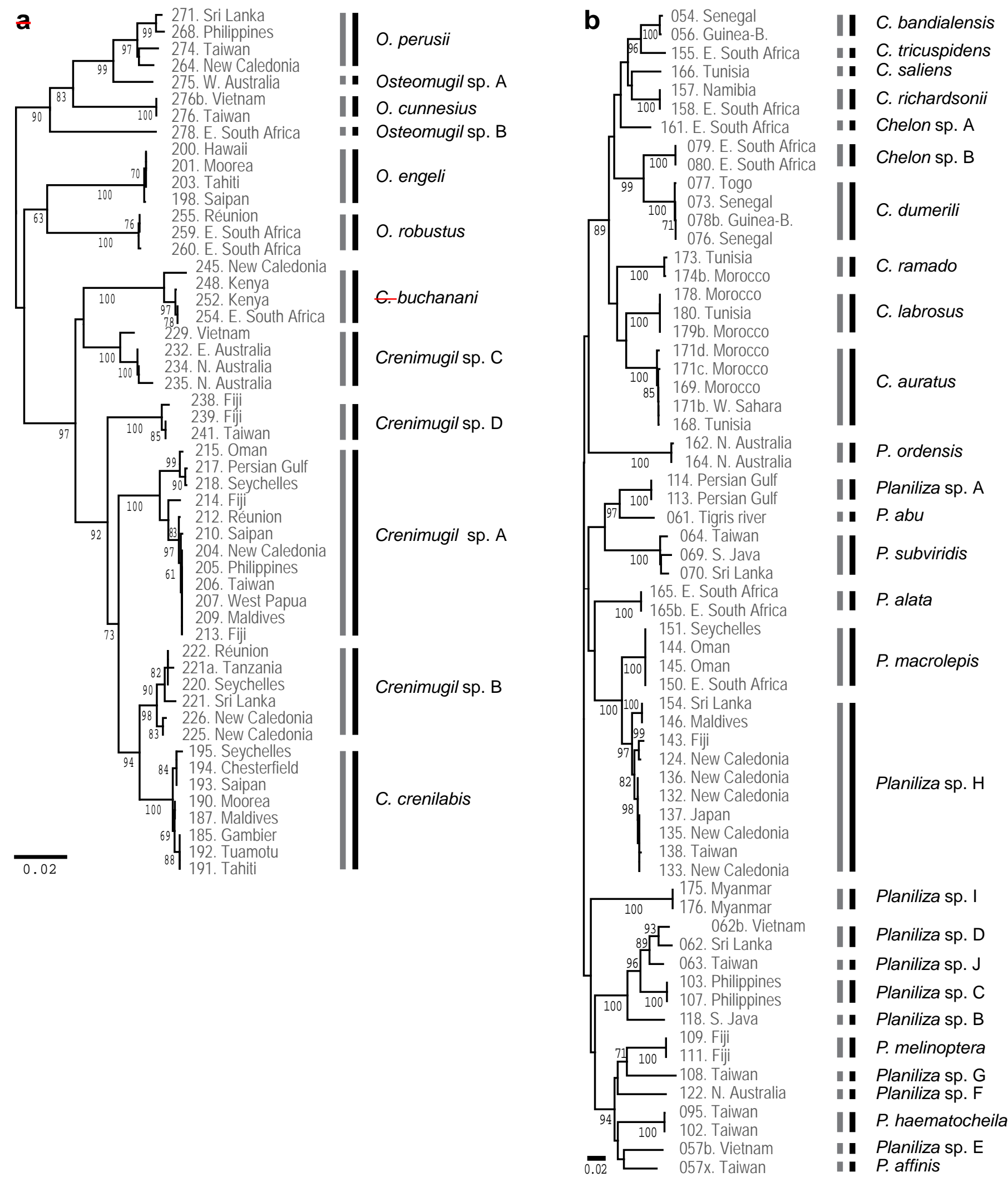

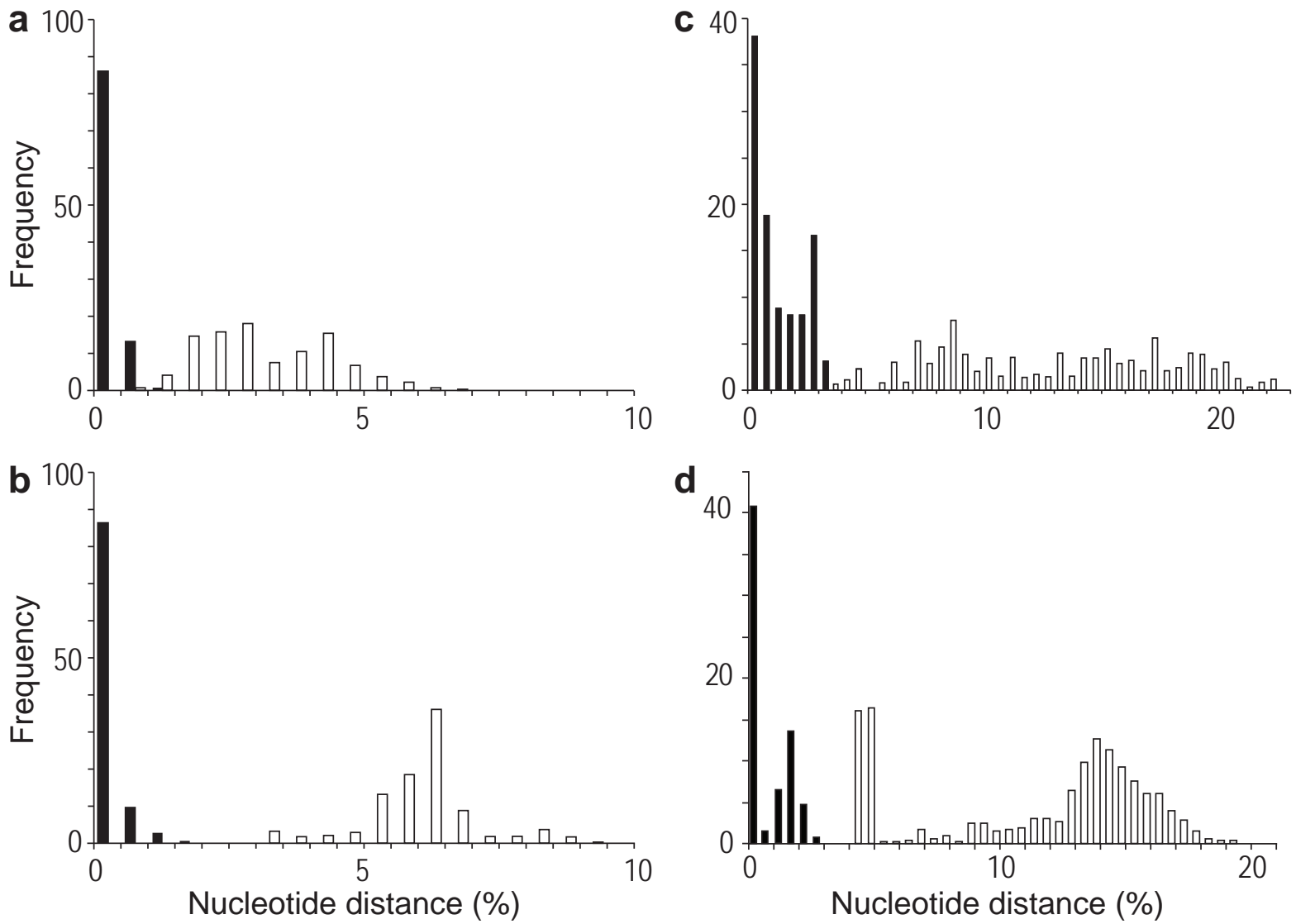
Supplementary material to:

\section{DNA barcoding grey mullets}

Jean-Dominique Durand · Nicolas Hubert · Kang-Ning Shen · Philippe Borsaף

Supplementary Table S1 and Figs. S1-S9 here appended. 


\section{References}

Aquino LM, Tango JM, Canoy RJ, Fontanilla IK, Basiao ZU, Ong PS, Quilang JP (2011) DNA barcoding of fishes of Laguna de Bay, Philippines. Mitochondr DNA 22:143-153

Aquilino SVL, Tango JM, Fontanilla IKC, Pagulayan RC, Basiao ZU, Ong PS, Quilang JP (2011) DNA barcoding of the ichthyofauna of Taal Lake, Philippines. Mol Ecol Resour 11:612-619

Asgharian H, Sahafi HH, Ardalan AA, Shekarriz S, Elahi E (2011) Cytochrome c oxidase subunit 1 barcode data of fish of the Nayband National Park in the Persian Gulf and analysis using meta-data flag several cryptic species. Mol Ecol Resour 11:461-472

Ashiq Ur Rahman M, Ajmal Khan S, Lyla PS, Durand J-D (2014) First record of Osteomugil perusii (Teleostei: Mugilidae) in Indian waters. Mar Biodiv Rec 7:e15

Ashiq Ur Rahman M, Ajmal Khan S, Lyla PS, Prasanna Kumar C (2013) DNA barcoding resolves taxonomic ambiguity in Mugilidae of Parangipettai waters (southeast coast of India). Turk J Fisheries Aquat Sci 13:321-330

Chen J, Li Y, Chen H, Yan B, Meng X (2015) The complete mitochondrial genome of the redeye mullet Liza haematocheila (Teleostei, Mugilidae). Mitochondr DNA 26:769-770

Costa FO, Landi M, Martins R, Costa MH, Costa ME, Carneiro M, Alves MJ, Steinke D, Carvalho GR (2012) A ranking system for reference libraries of DNA barcodes: application to marine fish species from Portugal. PLoS One 7:e35858

Durand J-D, Borsa P (2015) Mitochondrial phylogeny of grey mullets (Acanthopterygii: Mugilidae) suggests high proportion of cryptic species. C R Biol 338:266-277

Durand J-D, Chen W-J, Shen K-N, Fu C, Borsa P (2012a) Genus-level taxonomic changes implied by the mitochondrial phylogeny of grey mullets (Teleostei: Mugilidae). C R Biol 335:687-697

Durand J-D, Shen K-N, Chen W-J, Jamandre BW, Blel H, Diop K, Nirchio M, Garcia de Leon FJ, Whitfield AK, Chang C-W, Borsa P (2012b) Systematics of the grey mullets (Teleostei: Mugiliformes: Mugilidae): Molecular phylogenetic evidence challenges two centuries of morphology-based taxonomy. Mol Phyl Evol 64:73-92

Geiger MF, Herder F, Monaghan MT, Almada V, Barbieri R, Bariche M, Berrebi P, Bohlen J, Casal-Lopez M, Delmastro GB, Denys GPJ, Dettai A, Doadrio I, Kalogianni E, Kärst H, Kottelat M, Kovačić M, Laporte M, Lorenzoni M, Marčić Z, Özuluğ M, Perdices A, Perea S, Persat H, Porcelotti S, Puzzi C, Robalo J, Šanda R, Schneider M, Šlechtová V, Stoumboudi M, Walter S, Freyhof J (2014) Spatial heterogeneity in the Mediterranean biodiversity hotspot affects barcoding accuracy of its freshwater fishes. Mol Ecol Resour 14:1210-1221

Heras S, Gonzalez Castro M, Roldán MI (2006) Mugil curema in Argentinean waters: Combined morphological and molecular approach. Aquaculture 261:473-478

Heras S, Roldán MI, Gonzalez Castro M (2009) Molecular phylogeny of Mugilidae fishes revised. Rev Fish Biol Fisheries 19:217-231

Hett AK, Nirchio M, Oliveira C, Siccha Ramirez RZ, Rossi AR, Sola L (2011) Karyotype characterization of Mugil incilis Hancock, 1830 (Mugiliformes: Mugilidae), including a description of an unusual co-localization of major and minor ribosomal genes in the family. Neotrop Ichthyol 9:109-112

Hubert N, Meyer CP, Bruggemann HJ, Guerin F, Komeno RJ, Espiau B, Causse R, Williams JT, Planes S (2012) Cryptic diversity in IndoPacific coral-reef fishes revealed by DNA-barcoding provides new support to the centre-of-overlap hypothesis. PLoS One 7:e28987

Keskin E, Atar HH (2013) DNA barcoding commercially important fish species of Turkey. Mol Ecol Resour 13:788-797

Khedkar GD, Jamdade R, Naik S, David L, Haymer D (2014) DNA barcodes for the fishes of the Narmada, one of India's longest rivers. PLoS One 9:e10146

Lara A, Ponce de Leon JL, Rodriguez R, Casane D, Cote G, Bernatchez L, Garcia-Machado E (2010) DNA barcoding of Cuban freshwater fishes: evidence for cryptic species and taxonomic conflicts. Mol Ecol Resour 10:421-430

Lakra WS, Verma MS, Goswami M, Lal KK, Mohindra V, Punia P, Gopalakrishnan A, Singh KV, Ward RD, Hebert P (2011) DNA barcoding Indian marine fishes. Mol Ecol Resour 11:60-71

Mabragaña E, Díaz de Astarloa JM, Hanner R, Zhang J, González Castro M (2011) DNA barcoding identifies Argentine fishes from marine and brackish waters. PLoS One 12:e28655

Mejia O, Leon-Romero Y, Soto-Galera E (2012) DNA barcoding of the ichthyofauna of Panuco-Tamesi complex: Evidence for taxonomic conflicts in some groups. Mitochondr DNA 23:471-476

Oliveira Ribeiro A de, Caires RA, Mariguela TC, Pereira LH, Hanner R, Oliveira C (2012) DNA barcodes identify marine fishes of Sao Paulo State, Brazil. Mol Ecol Resour 12:1012-1020

Page TJ, Hughes JM (2010) Comparing the performance of multiple mitochondrial genes in the analysis of Australian freshwater fishes. J Fish Biol 77:2093-2122

Shen K-N, Jamandre BW, Hsu C-C, Tzeng W-N, Durand J-D (2011) Plio-Pleistocene sea level and temperature fluctuations in the northwestern Pacific promoted speciation in the globally-distributed flathead mullet Mugil cephalus. BMC Evol Biol 11:83

Siccha-Ramirez R, Menezes NA, Nirchio M, Foresti F, Oliveira C (2014) Molecular identification of mullet species of the Atlantic South Caribbean and South America and the phylogeographic analysis of Mugil liza. Rev Fish Sci Aquaculture 22:86-96

Sparks JS, Smith WL (2004) Phylogeny and biogeography of the Malagasy and Australasian rainbowfishes (Teleostei: Melanotaenioidei): Gondwanan vicariance and evolution in freshwater. Mol Phyl Evol 33:719-734

Ward RD, Holmes BH (2007) An analysis of nucleotide and amino acid variability in the barcode region of cytochrome c oxidase I (cox1) in fishes. Mol Ecol Notes 7:899-907

Weigt LA, Baldwin CC, Driskell A, Smith DG, Ormos A, Reyier EA (2012) Using DNA barcoding to assess Caribbean reef fish biodiversity: expanding taxonomic and geographic coverage. PLoS One 7:e41059

Zhang J (2011) Species identification of marine fishes in China with DNA barcoding. Evidence-Based Compl Altern Med 978253

Zhang J-B, Hanner R (2011) DNA barcoding is a useful tool for the identification of marine fishes from Japan. Biochem Syst Ecol 39:31-42

Zhang J, Hanner R (2012) Molecular approach to the identification of fish in the South china sea. PLoS One 7:e30621 


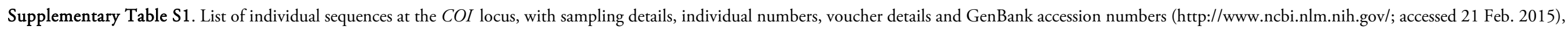

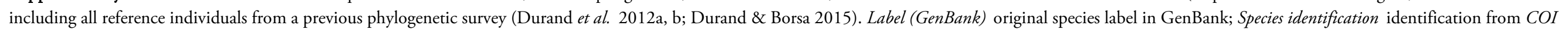

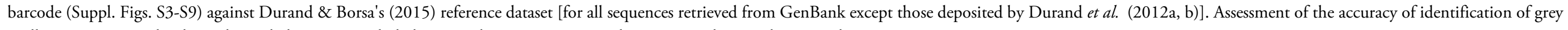
mullet specimens used in barcoding, phylogenetic, and phylogeographic surveys: $M$ match; $X$ mismatch; $u$ undetermined

\begin{tabular}{|c|c|c|c|c|c|c|c|c|c|c|}
\hline $\begin{array}{l}\text { Sequence no } \\
\text { (GenBank) }\end{array}$ & Specimen no. & Tissue voucher no. & $\begin{array}{l}\text { Sampling details } \\
\text { Location }\end{array}$ & Date & Collector(s) & Reference & Survey tvpe & Label(GenBank) & Species identification & Match \\
\hline$\overline{A Y 655531}$ & - & - & Panama City, Florida, USA & - & - & Sparks and Smith 2004 & Phylogeny & Mugil cephalus & Mugil sp. C & M \\
\hline DQ441603 & IMCUR & - & - & - & - & Heras et al. 2006 & Phylogeography & Mugil curema & Mugil sp. & u \\
\hline DQ441604 & 2MCUR & - & - & - & - & Heras et al. 2006 & Phylogeography & Mugil curema & Mugil curema & M \\
\hline DQ441605 & 3MCUR & - & - & - & - & Heras et al. 2006 & Phylogeography & Mugil curema & Mugil curema & M \\
\hline DQ441607 & 5MCUR & - & - & - & - & Heras et al. 2006 & Phylogeography & Mugil curema & Mugil curema & M \\
\hline DQ441608 & 6MCEP & - & Mediterranean $\left(41^{\circ} 50^{\prime} \mathrm{N}, 03^{\circ} 08^{\prime} \mathrm{E}\right)$ & - & - & Heras et al. 2006 & Phylogeography & Mugil cephalus & Mugil cephalus & M \\
\hline DQ441609 & 7MCEP & - & Mediterranean $\left(41^{\circ} 50^{\prime} \mathrm{N}, 03^{\circ} 08^{\prime} \mathrm{E}\right)$ & - & - & Heras et al. 2006 & Phylogeography & Mugil cephalus & Mugil cephalus & M \\
\hline DQ441610 & 8MCEP & - & Mediterranean $\left(41^{\circ} 50^{\prime} \mathrm{N}, 03^{\circ} 08^{\prime} \mathrm{E}\right)$ & - $\quad$ & - & Heras et al. 2006 & Phylogeography & Mugil cephalus & Mugil cephalus & M \\
\hline EF607446 & FSCS304-06 & GD 9087015 & South China Sea, China $\left(21.43^{\circ} \mathrm{N} 110.13^{\circ} \mathrm{E}\right)$ & 10 Aug. 2006 & J. Zhang & Zhang 2011 & Barcoding & Liza sp. & Planiliza subviridis & u \\
\hline EF607447 & FSCS303-06 & GD 9087014 & South China Sea, China $\left(21.42^{\circ} \mathrm{N} 110.17^{\circ} \mathrm{E}\right)$ & 10 Aug. 2006 & J. Zhang & Zhang 2011 & Barcoding & Liza sp. & Planiliza subviridis & u \\
\hline EF607615 & FSCS251-06 & GD 9083012 & South China Sea, China & & J. Zhang & Zhang 2011 & Barcoding & Valamugil engeli & Planiliza affinis & $\mathrm{x}$ \\
\hline$E F 609279^{a}$ & CSIRO H3962-06 & BW-A1175 & unknown location, Australia & 13 Apr. 1995 & G. Yearsley & Ward and Holmes 2007 & Barcoding & Aldrichetta forsteri & Aldrichetta forsteri & . \\
\hline EF609494 ${ }^{a}$ & CSIRO-H4596-01 & BW-A1182 & Mackay harbour boat ramp, Queensland, Australia $\left(21.28^{\circ} \mathrm{S} 149.30^{\circ} \mathrm{E}\right)$ & 30 May 1997 & CRIMP & Ward and Holmes 2007 & Barcoding & Moolgarda seheli & Crenimugil sp. C & M \\
\hline EF609544 & - & WL-M378 & Kerala, India $\left(9.97^{\circ} \mathrm{N} 76.23^{\circ} \mathrm{E}\right)$ & 27 Mar. 2006 & - & Lakra et al. 2011 & Barcoding & Chelon macrolepis & Planiliza subviridis & $\mathrm{x}$ \\
\hline EF609545 & - & WL-M376 & Kerala, India $\left(9.97^{\circ} \mathrm{N} 76.23^{\circ} \mathrm{E}\right)$ & 27 Mar. 2006 & - & Lakra et al. 2011 & Barcoding & Chelon macrolepis & Planiliza subviridis & $\mathrm{x}$ \\
\hline EF609546 & - & WL-M375 & Kerala, India $\left(9.97^{\circ} \mathrm{N} 76.23^{\circ} \mathrm{E}\right)$ & 27 Mar. 2006 & - & Lakra et al. 2011 & Barcoding & Chelon macrolepis & Planiliza subviridis & $\mathrm{x}$ \\
\hline EF609547 & - & WL-M374 & Kerala, India $\left(9.97^{\circ} \mathrm{N} 76.23^{\circ} \mathrm{E}\right)$ & 26 Mar. 2006 & - & Lakra et al. 2011 & Barcoding & Chelon macrolepis & Planiliza subviridis & $\mathrm{x}$ \\
\hline EU014262 & - & WL-M785 & India & - & - & Lakra et al. 2011 & Barcoding & Liza tade & Osteomugil sp. & $\mathrm{x}$ \\
\hline EU014263 & - & WL-M786 & India & - & - & Lakra et al. 2011 & Barcoding & Liza tade & Osteomugil sp. & $x$ \\
\hline EU014264 & - & WL-M787 & India & - & - & Lakra et al. 2011 & Barcoding & Liza tade & Osteomugil sp. & $x$ \\
\hline EU014265 & - & WL-M788 & India & - & - & Lakra et al. 2011 & Barcoding & Liza tade & Osteomugil sp. & $x$ \\
\hline EU074483 & FARG331-07 & INIDEP-T 0330 & Argentina $\left(37.32^{\circ} \mathrm{S} 57.19^{\circ} \mathrm{W}\right)$ & 10 Oct. 2006 & M. Gonzalez Castro & Mabragana et al. 2011 & Barcoding & Mugil platanus & Mugil liza & M \\
\hline EU392233 & $\mathrm{Cl}$ & - & Italy & - & - & - & Phylogeny & Chelon labrosus & Chelon labrosus & M \\
\hline EU392234 & La_A & - & Azov Sea, Russia & - & - & & Phylogeny & Liza aurata & Chelon auratus & M \\
\hline EU392235 & La_M & - & Italy & - & - & - & Phylogeny & Liza aurata & Chelon auratus & M \\
\hline EU392236 & $\mathrm{Ch}_{-}^{-} \mathrm{A}$ & - & Azov Sea, Russia & - & - & - & Phylogeny & Liza haematocheila & Planiliza haematocheila & M \\
\hline EU392237 & $\mathrm{Ch}_{-} \mathrm{J}$ & - & Japan Sea, Russia & - & - & - & Phylogeny & Liza haematocheila & Planiliza haematocheila & M \\
\hline EU392239 & $\mathrm{Lm}$ & - & Taiwan & - & - & - & Phylogeny & Chelon macrolepis & Planiliza sp. H & M \\
\hline EU392240 & Lr & - & Italy & - & - & - & Phylogeny & Liza ramada & Chelon ramado & M \\
\hline EU392241 & Ls & - & Italy & - & - & - & Phylogeny & Liza saliens & Chelon saliens & M \\
\hline EU392242 & Lsub & - & Taiwan & - & - & - & Phylogeny & Liza subviridis & Planiliza affinis & $\mathrm{x}$ \\
\hline EU392243 & Mc_A & - & Azov Sea, Russia & - & - & - & Phylogeny & Mugil cephalus & Mugil cephalus & M \\
\hline EU392246 & $\mathrm{Mc}_{-}^{\top}{ }^{\top}$ & - & Taiwan & - & - & - & Phylogeny & Mugil cephalus & Mugil sp. L & M \\
\hline EU392247 & $\mathrm{Vc}^{-}$ & - & Taiwan & - & - & - & Phylogeny & Moolgarda cunnesius & Crenimugil sp. D & u \\
\hline EU489714 & - & SIO:98-1 & California, USA & - & - & - & Barcoding & Mugil curema & Mugil sp. 0 & M \\
\hline EU595084 & - & MBCSC:Z711229 & South China Sea, China $\left(20.05^{\circ} \mathrm{N} 111.48^{\circ} \mathrm{E}\right)$ & 28 Oct. 2007 & J. Zhang & - & Barcoding & Mugil cephalus & Mugil sp. I & M \\
\hline EU595085 & - & MBCSC:Z711228 & South China Sea, China $\left(21.09^{\circ} \mathrm{N} 112.55^{\circ} \mathrm{E}\right)$ & 28 Oct. 2007 & J. Zhang & - & Barcoding & Mugil cephalus & Mugil sp. I & M \\
\hline EU595338 & - & MBCSC:Z711255 & South China Sea, China $\left(21.07^{\circ} \mathrm{N} 111.56^{\circ} \mathrm{E}\right)$ & 30 Oct. 2007 & J. Zhang & - & Barcoding & Valamugil cunnesius & Osteomugil cunnesius & M \\
\hline EU595339 & - & MBCSC:Z711254 & South China Sea, China $\left(21.07^{\circ} \mathrm{N} 111.56^{\circ} \mathrm{E}\right)$ & 30 Oct. 2007 & J. Zhang & - & Barcoding & Valamugil cunnesius & Osteomugil cunnesius & M \\
\hline EU595340 & - & MBCSC:Z711253 & South China Sea, China $\left(19.68^{\circ} \mathrm{N} 112.77^{\circ} \mathrm{E}\right)$ & 30 Oct. 2007 & J. Zhang & - & Barcoding & Valamugil cunnesius & Osteomugil cunnesius & M \\
\hline EU595341 & - & MBCSC:Z711252 & South China Sea, China $\left(19.68^{\circ} \mathrm{N} 112.77^{\circ} \mathrm{E}\right)$ & 30 Oct. 2007 & J. Zhang & - & Barcoding & Valamugil cunnesius & Osteomugil cunnesius & M \\
\hline EU595342 & - & MBCSC:Z711251 & South China Sea, China $\left(20.52^{\circ} \mathrm{N} 110.98^{\circ} \mathrm{E}\right)$ & 30 Oct. 2007 & J. Zhang & - & Barcoding & Valamugil cunnesius & Osteomugil cunnesius & M \\
\hline EU595343 & - & MBCSC:Z711250 & South China Sea, China $\left(20.52^{\circ} \mathrm{N} 110.98^{\circ} \mathrm{E}\right)$ & 30 Oct. 2007 & J. Zhang & - $\quad 20$ & Barcoding & Valamugil cunnesius & Osteomugil cunnesius & M \\
\hline EU715451 & 4Mcep & - & Galveston Bay, USA & - & - & Heras et al. 2009 & Phylogeny & Mugil cephalus & Mugil sp. B & M \\
\hline EU715452 & 5Mcep & - & Galveston Bay, USA & - & - & Heras et al. 2009 & Phylogeny & Mugil cephalus & Mugil sp. B & M \\
\hline EU715456 & 9Mpl & - & Montevideo, Uruguay & - & - & Heras et al. 2009 & Phylogeny & Mugil platanus & Mugil liza & M \\
\hline EU715457 & $10 \mathrm{Mpl}$ & - & Samborombon Bay, Argentina & - & - & Heras et al. 2009 & Phylogeny & Mugil platanus & Mugil liza & M \\
\hline EU715458 & 11Mpl & - & Viedma, Argentina & - & - & Heras et al. 2009 & Phylogeny & Mugil platanus & Mugil liza & M \\
\hline
\end{tabular}




\begin{tabular}{|c|c|}
\hline EU715459 & $12 \mathrm{Mpl}$ \\
\hline EU715460 & $13 \mathrm{Mpl}$ \\
\hline EU715461 & $14 \mathrm{Mpl}$ \\
\hline EU715462 & 15Mli \\
\hline EU715463 & 21Mcur \\
\hline EU715464 & 22Mcur \\
\hline EU715465 & 23Mcur \\
\hline EU715466 & 24Lau \\
\hline EU715467 & 25Lau \\
\hline EU715468 & 26Lau \\
\hline EU715470 & 28Lsal \\
\hline EU715471 & 29Lsal \\
\hline EU715472 & $30 \mathrm{Chl}$ \\
\hline EU715473 & $31 \mathrm{CH} 1$ \\
\hline EU715474 & $32 \mathrm{Chl}$ \\
\hline FJ238048 & - \\
\hline FJ347895 & - \\
\hline FJ347896 & - \\
\hline FJ347897 & - \\
\hline FJ347898 & - \\
\hline FJ347899 & - \\
\hline FJ347967 & - \\
\hline FJ384677 & IOBML1 \\
\hline FJ918950 & - \\
\hline FN545593 & BC-110 \\
\hline FN545594 & BC-115 \\
\hline FN600159 & - \\
\hline FN600160 & - \\
\hline GU224562 & - \\
\hline GU224563 & - \\
\hline GU225390 & - \\
\hline GU225391 & - \\
\hline GU225392 & - \\
\hline GU225393 & - \\
\hline GU225394 & - \\
\hline GU225395 & - \\
\hline GU225396 & - \\
\hline GU225397 & - \\
\hline GU225398 & - \\
\hline GU225399 & - \\
\hline GU225400 & - \\
\hline GU225401 & - \\
\hline GU225402 & - \\
\hline GU225403 & - \\
\hline GU225404 & - \\
\hline GU225405 & - \\
\hline GU225406 & - \\
\hline GU260664 & 08KP1_HT2 \\
\hline GU260665 & 05KS21_HT4 \\
\hline GU260666 & 05KS22_HT1C \\
\hline GU260667 & 07KS6_HT1 \\
\hline GU260668 & 05KL32_HT5 \\
\hline GU260669 & 07KL12_HT6 \\
\hline GU260670 & 07KL33_HT3 \\
\hline GU260671 & 07KS37_HT11 \\
\hline GU260672 & 06KL23_HT12 \\
\hline GU260673 & 07KL27_HT7 \\
\hline GU260674 & 05KS19_HT8 \\
\hline GU260675 & 05KL42_HT9 \\
\hline GU260676 & 07KS4_HT13 \\
\hline GU260677 & 05KL7_HT15 \\
\hline GU260678 & YOKOS̄UKA9 \\
\hline
\end{tabular}

San Lorenzo lagoon, Argentina San Lorenzo lagoon, Argentina San Lorenzo lagoon, Argentina Zaza, Cuba

Mar Chiquita lagoon, Argentina

Mar del Plata, Argentina

Argentina

Palamos, Spain

Palamos, Spain

Moulay Bousselham lagoon Morocco

Moulay Bousselham lagoon, Morocco

Ter Vell lagoon, Spain

Ter Vell lagoon, Spain NBFGR:MC174

South China Sea, China $\left(20.52^{\circ} \mathrm{N} 110.98^{\circ} \mathrm{E}\right)$

umbai, India $\left(19.02^{\circ} \mathrm{N} 72.46^{\circ} \mathrm{E}\right)$

Mumbi,

Mumbai, na (19.02 $\left.72.46^{\circ}\right)$

Muma,

Kochi, India $\left(10.02^{\circ} \mathrm{N} 76.13^{\circ} \mathrm{E}\right)$

Bay of Bengal, India

14 Apr. 2008

SV_1

MFP:18.000247

年, Cuba

Manglarito stream, Baracoa, Guantanamo, Cuba

Red Sea, Egyp

West Cancun St.5 G.G., Quintana Roo, Mexico $\left(21.37^{\circ} \mathrm{N} 86.14^{\circ} \mathrm{W}\right) \quad 01$ jan. $2007 \quad$ I. Castellanos Osorio

West Cancun St.5 G.G., Quintana Roo, Mexico $\left(21.37^{\circ} \mathrm{N} 86.14^{\circ} \mathrm{W}\right)$ - 01 jan. $2007 \quad$ I. Castlonos Osio

Puente Xcalak, Quintana Roo, Mexico

01 Dec. 2005 R. Herrera et

MFL1507

MX160

MX659

MX663
MX660

MX662

XX61

MX668

ЕСО-СH-P54938

CO-CH-P5493A

ECO-CH-P5493C

MX159
MX664
MX667

MX667

MX666

ECO-CH-P5688d

ECO-CH-P5688

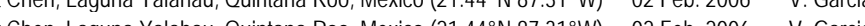

Pt Chen, Laguna Yalahau, Quintana Roo, Mexico $\left(21.44^{\circ} 87.31^{\circ} \mathrm{W}\right)$ 02 Feb. $2006 \quad$ V. Garcia

Pt Chen La

Pt Chen Laguna Yalahau, Quintana Roo, Mexico (21.44 N87.31 W) 02 Feb. 2006 V. Garcia

Pt Chen, Laguna Yalahau, Quintana Ro, Mexico $\left(21.44^{\circ} \mathrm{N} 87.31^{\circ} \mathrm{W}\right) 02 \mathrm{Feb} .2006$ V Garcia

PtChen, Laguna Yalahau, Quintana Roo, Mexico $\left(21.44^{\circ} \mathrm{N} 87.31^{\circ} \mathrm{W}\right)$ 02 Feb. 2006 V. Garcia

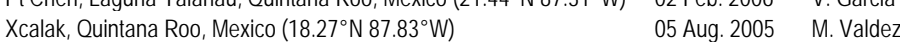

Xcalak, Quintana Roo, Mexico $\left(18.27^{\circ} \mathrm{N} 87.83^{\circ} \mathrm{W}\right) \quad 05$ Aug. 2005 M. Valdez

Xcalak, Quintana Roo, Mexico $\left(18.27^{\circ} \mathrm{N} 87.83^{\circ} \mathrm{W}\right) \quad 05$ Aug. $2005 \quad$ M. Valdez

Puente Xcalak, Quintana Roo, Mexico $\left(18.28^{\circ} \mathrm{N} 87.83^{\circ} \mathrm{W}\right) \quad 01 \mathrm{Dec} .2005 \quad$ R. Herrera et

Pt Chen, Laguna Yalahau, Quintana Roo, Mexico $\left(21.44^{\circ} \mathrm{N} 87.31^{\circ} \mathrm{W}\right) \quad 02$ Feb. $2006 \quad$ V. Garcia

PtChen, Laguna Yalahau, Quintana Roo, Mexico (21.44\% 87.31\%) 02 Feb. $2006 \quad$ M. Valdez

Pt Chen, Laguna Yalahau, Quintana Roo, Mexico $\left(21.44^{\circ} \mathrm{N} 87.31^{\circ} \mathrm{W}\right) \quad 02$ Feb. $2006 \quad$ V. Garcia

Xcalak, Quintana Roo, Mexico $\left(18.27 \mathrm{~N}^{\circ} 87.83^{\circ} \mathrm{W}\right) \quad 05$ Aug. $2005 \quad$ M. Valdez

Xcalak, Quintana Roo, Mexico $\left(18.27^{\circ} \mathrm{N} 87.83^{\circ} \mathrm{W}\right) \quad 05$ Aug. $2005 \quad$ M. Valdez

Kaoping Estuary, Taiwan

Kaohsiung, Taiwan

Kaohsiung, Taiwan

Kaohsiung, Taiwan

Keelung, Taiwan

Keelung, Taiwan

Keelung, Taiwan

Kaohsiung, Taiwan

Keelung, Taiwan

Keelung, Taiwan

Kaohsiung, Taiwan

Keelung, Taiwan

Kaohsiung, Taiwa

Keelung, Taiwan
Yokosuka, Japan

K.-N. Shen
K.-N. Shen

K.-N. She

K.-N. Shen

K.-N. Shen

K.-N. Shen

K.-N. Shen

K.-N. She

K.-N. She

K.-N. Shen

K.-N. She

K.-N. She

K.-N. Shen

K.-N. She

K.-N. She

S. Chow
Heras et al. 2009

Heras et al. 2009

Heras et al. 2009

Heras et al. 2009

Heras et al. 2009

Heras et al. 2009

Heras et al. 2009

Heras et al. 2009

Heras et al. 2009

Heras et al. 2009

Heras et al. 2009

Heras et al. 2009

Heras et al 2009

Lakra et al. 2011

Lakra et al. 201

Lakra et al. 201

Lakra et al. 201

Lakra et al. 2011

-

Lara et al. 2010

hen et al. 201

Shen et al. 201

Shen et al. 201

Shen et al. 2011

Shen et al. 201

Shen et al. 2011

Shen et al. 201

Shen et al. 201

Shen et al. 2011

Shen et al. 201

Shen et al. 201

Shen et al. 201

Shen et al. 201

Shen et al. 2011

\begin{tabular}{|c|c|c|}
\hline Phylogeny & Mugil platanus & Mugil liza \\
\hline Phylogeny & Mugil platanus & Mugil liza \\
\hline Phylogeny & Mugil platanus & Mugil liza \\
\hline Phylogeny & Mugil liza & Mugil liza \\
\hline Phylogeny & Mugil curema & Mugil curema \\
\hline Phylogeny & Mugil curema & Mugil curema \\
\hline Phylogeny & Mugil curema & Mugil rubrioculus \\
\hline Phylogeny & Liza aurata & Chelon auratus \\
\hline Phylogeny & Liza aurata & Chelon auratus \\
\hline Phylogeny & Liza aurata & Chelon auratus \\
\hline Phylogeny & Liza saliens & Chelon saliens \\
\hline Phylogeny & Liza saliens & Chelon saliens \\
\hline Phylogeny & Chelon labrosus & Chelon labrosus \\
\hline Phylogeny & Chelon labrosus & Chelon labrosus \\
\hline Phylogeny & Chelon labrosus & Chelon labrosus \\
\hline Barcoding & Valamugil cunnesius & Osteomugil cunnesius \\
\hline Barcoding & Mugil cephalus & Planiliza sp. D \\
\hline Barcoding & Mugil cephalus & Planiliza sp. D \\
\hline Barcoding & Mugil cephalus & Planiliza sp. D \\
\hline Barcoding & Mugil cephalus & Planiliza sp. D \\
\hline Barcoding & Mugil cephalus & Planiliza sp. D \\
\hline Barcoding & Chelon macrolepis & Planiliza subviridis \\
\hline Barcoding & Liza parsia & Planiliza sp. H \\
\hline Phylogeny & Mugil trichodon & Mugil sp. R \\
\hline Barcoding & Agonostomus monticola & Dajaus monticola \\
\hline Barcoding & Agonostomus monticola & Dajaus monticola \\
\hline Barcoding & Liza carinata & Planiliza sp. $H$ \\
\hline Barcoding & Crenimugil crenilabis & Crenimugil sp. B \\
\hline Barcoding & Mugil cephalus & Mugil trichodon \\
\hline Barcoding & Mugil cephalus & Mugil trichodon \\
\hline Barcoding & Mugil sp. & Mugil liza \\
\hline Barcoding & Mugil cephalus & Mugil trichodon \\
\hline Barcoding & Mugil cephalus & Mugil trichodon \\
\hline Barcoding & Mugil cephalus & Mugil trichodon \\
\hline Barcoding & Mugil cephalus & Mugil trichodon \\
\hline Barcoding & Mugil cephalus & Mugil trichodon \\
\hline Barcoding & Mugil curema & Mugil rubrioculus \\
\hline Barcoding & Mugil curema & Mugil rubrioculus \\
\hline Barcoding & Mugil curema & Mugil rubrioculus \\
\hline Barcoding & Mugil curema & Mugil rubrioculus \\
\hline Barcoding & Mugil curema & Mugil rubrioculus \\
\hline Barcoding & Mugil liza & Mugil sp. B \\
\hline Barcoding & Mugil trichodon & Mugil curema \\
\hline Barcoding & Mugil trichodon & Mugil curema \\
\hline Barcoding & Mugil trichodon & Mugil curema \\
\hline Barcoding & Mugil trichodon & Mugil curema \\
\hline Barcoding & Mugil trichodon & Mugil curema \\
\hline Phylogeography 1 & Mugil cephalus & Mugil sp. C \\
\hline Phylogeography & Mugil cephalus & Mugil sp. C \\
\hline Phylogeography 1 & Mugil cephalus & Mugil sp. C \\
\hline Phylogeography & Mugil cephalus & Mugil sp. C \\
\hline Phylogeography & Mugil cephalus & Mugil sp. C \\
\hline Phylogeography & Mugil cephalus & Mugil sp. C \\
\hline Phylogeography 1 & Mugil cephalus & Mugil sp. C \\
\hline Phylogeography 1 & Mugil cephalus & Mugil sp. C \\
\hline Phylogeography & Mugil cephalus & Mugil sp. C \\
\hline Phylogeography & Mugil cephalus & Mugil sp. C \\
\hline Phylogeography & Mugil cephalus & Mugil sp. C \\
\hline Phylogeography & Mugil cephalus & Mugil sp. C \\
\hline Phylogeography & Mugil cephalus & Mugil sp. C \\
\hline Phylogeography & Mugil cephalus & Mugil sp. C \\
\hline Phylogeography & Mugil cephalus & Mugil sp. C \\
\hline
\end{tabular}




\begin{tabular}{|c|c|c|c|}
\hline GU260679 & 08KP2_H25 & - & Kaoping Estuary, Taiwan \\
\hline GU260680 & 08KP31_H16 & - & Kaoping Estuary, Taiwan \\
\hline GU260681 & 05KP3_Н̈T24 & - & Kaoping Estuary, Taiwan \\
\hline GU260682 & 05KL6_HT27 & - & Keelung, Taiwan \\
\hline GU260683 & OKINAWWA17_HT26 & - & Okinawa Is., Japan \\
\hline GU260684 & 08KP22_HT18 & - & Kaoping Estuary, Taiwan \\
\hline GU260685 & 08KP38_HT21 & - & Kaoping Estuary, Taiwan \\
\hline GU260686 & 07KL1_HT19 & - & Keelung, Taiwan \\
\hline GU260687 & 07KL3_HT22 & - & Keelung, Taiwan \\
\hline GU260688 & 07KL41_HT20 & - & Keelung, Taiwan \\
\hline GU260689 & PHILI6_HT23 & - & Luzon, Philippines \\
\hline GU260690 & 08KP35_HT28 & . & Kaoping Estuary, Taiwan \\
\hline GU260691 & 07KP9_HT29 & - & Kaoping Estuary, Taiwan \\
\hline GU260692 & PEARL3_HT30 & - & Pearl River, China \\
\hline GU260693 & 07KL21_HT17 & - & Keelung, Taiwan \\
\hline GU260694 & 08KP9_НТT32 & - & Kaoping Estuary, Taiwan \\
\hline GU260695 & 05KP2_HT31 & - & Kaoping Estuary, Taiwan \\
\hline GU260696 & PEARL7_HT33 & . & Pearl River, China \\
\hline GU260697 & SHANTOU7_HT34 & - & Shantou, China \\
\hline GU440409 & 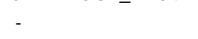 & MFC108 & S of La Bocana, Baja California S, Mexico $\left(26.75^{\circ} \mathrm{N} 114.25^{\circ} \mathrm{W}\right)$ \\
\hline GU702392 & - & LBP-35141 & Sao Paulo, Brazil \\
\hline GU702394 & - & LBP-35143 & Sao Paulo, Brazil \\
\hline GU702395 & - & LBP-35139 & Sao Paulo, Brazil \\
\hline GU702397 & . & LBP-35142 & Sao Paulo, Brazil \\
\hline HM006970 & . & GU_AW418 & Southeastern Queensland, Australia \\
\hline HM180700 & - & NSMK:PI-000415 & Korea \\
\hline HM208823 & 2Cba & - & Senegal and Bijagos \\
\hline HM208824 & 3Cba & MNHN 2009-0790-94 & Saloum estuary, Senegal \\
\hline HM208825 & 6Cba & MNHN 2009-0790-94 & Saloum estuary, Senegal \\
\hline HM208826 & 8Ldu & - & West Africa \\
\hline HM208827 & 9Ldu & . & West Africa \\
\hline HM208828 & 19Lfa & . & Saloum estuary, Senegal \\
\hline HM208829 & 22Lfa & - & West Africa \\
\hline HM208830 & $26 \mathrm{Lfa}$ & - & Lomé fish market, Togo \\
\hline HM208831 & $27 \mathrm{Lgr}$ & - & West Africa \\
\hline HM208832 & 29Lgr & - & Saloum estuary, Senegal \\
\hline HM208833 & Ls & . & La Goulette, Tunisia \\
\hline HM208834 & 38La & - & Morocco \\
\hline HM208835 & 41La & - & Agadir, Morocco \\
\hline HM208836 & 43La & - & North East Atlantic, Mediterranean Sea \\
\hline HM208837 & $45 \mathrm{Cla}$ & - & North East Atlantic, Mediterranean Sea \\
\hline HM208838 & $46 \mathrm{Cla}$ & - & North East Atlantic, Mediterranean Sea \\
\hline HM208839 & 50Lra & . & North East Atlantic, Mediterranean Sea \\
\hline HM208840 & 52Lra & . & Morocco \\
\hline HM208841 & $56 \mathrm{Mba}$ & - & West Africa \\
\hline HM208842 & $60 \mathrm{Mba}$ & - & Ebrie Lagoon, Ivory Coast \\
\hline HM208843 & 63Mba & - & Ebrie Lagoon, Ivory Coast \\
\hline HM208844 & 64NEMce & - & North East Atlantic, Mediterranean Sea \\
\hline HM208845 & 67NEMce & - & Merja Zerga, Morocco \\
\hline HM208846 & 74WAMce & - & West Africa \\
\hline HM208847 & 75WAMce & . & West Africa \\
\hline HM208848 & 81WAMce & - & Cunene River, Angola \\
\hline HM208849 & 84WAMce & - & Nokoue Lagoon, Benin \\
\hline HM208850 & 87WAMce & - & Iwic, Mauritania \\
\hline HM208851 & 99мси & - & West Africa \\
\hline HM208852 & 104Mcu & . & Nokoue Lagoon, Benin \\
\hline HM208853 & $118 \mathrm{Mca}$ & - & Morocco and Senegal \\
\hline HM208854 & $122 \mathrm{Mca}$ & - & Lome fish market, Togo \\
\hline HM208855 & Ola & - & La Goulette, Tunisia \\
\hline HQ024977 & - & FDA 4-33 & Market sample, Washington DC, USA \\
\hline HQ024978 & - & FDA 3-33 & Market sample, Washington DC, USA \\
\hline HQ024979 & - & FDA 2-33 & Market sample, Washington DC, USA \\
\hline
\end{tabular}

\begin{tabular}{|c|c|}
\hline K.-N. Shen & Shen et al. 2011 \\
\hline K.-N. Shen & Shen et al. 2011 \\
\hline K.-N. Shen & Shen et al. 2011 \\
\hline K.-N. Shen & Shen et al. 2011 \\
\hline - & Shen et al. 2011 \\
\hline K.-N. Shen & Shen et al. 2011 \\
\hline K.-N. Shen & Shen et al. 2011 \\
\hline K.-N. Shen & Shen et al. 2011 \\
\hline K.-N. Shen & Shen et al. 2011 \\
\hline K.-N. Shen & Shen et al. 2011 \\
\hline B.W. Jamandre & Shen et al. 2011 \\
\hline K.-N. Shen & Shen et al. 2011 \\
\hline K.-N. Shen & Shen et al. 2011 \\
\hline X. Nie & Shen et al. 2011 \\
\hline K.-N. Shen & Shen et al. 2011 \\
\hline K.-N. Shen & Shen et al. 2011 \\
\hline K.-N. Shen & Shen et al. 2011 \\
\hline X. Nie & Shen et al. 2011 \\
\hline & Shen et al. 2011 \\
\hline
\end{tabular}

Phylogeography Mugil cephalus Phylogeography Mugil cephalus Phylogeography Mugil cephalus Phylogeography Mugil cephalus Phylogeography Mugil cephalus Phylogeography Mugil cephalus Phylogeography Mugil cephalus Mugil cephalus Mugil cephalus Phlogegraphy Phylogegraphy Murilcephalus Phylogeography Mugil cephalus Phylogeography Muril cephalus Phylogeography Murilcephalus Phylogegranhy Musilcephalus Phylogeography Muril cephalus Phylogeography Mugil cephalus Phylogeography Mugil cephalus Barcoding Mugil curema

01 Jan. $1998 \quad$ D. Talley, P. Dayton

Oliveira Ribeiro et al. 2012 Oliveira Ribeiro et al. 2012 Oliveira Ribeiro et al. 2012 arcoding Barcoding Oliveira Ribeiro et al. 2012

Barcoding Barcoding Barcoding Phlogeny Phlogeny Phylogeny Phylogeny Phylogeny

Phylogeny

Phylogeny

Phylogeny

Phylogeny

Phylogeny

Phylogeny

Phylogeny

Phylogeny

Phylogeny

Phylogeny

Phylogeny

Phylogeny

Phylogeny

Phylogeny

Phylogeny

Phylogeny

Phylogeny

Phylogeny

Phylogeny

Phylogeny

Phylogeny

Phylogeny

Phylogeny

Phylogeny

Phylogeny

Phylogeny

Phylogeny

Phylogeny

Barcoding

Barcoding
Barcoding
Mugil curema

Mugil liza

Mugil liza

Mugil liza

Liza haematocheil

Liza bandialensis

Liza bandialensis

Liza bandialensis

Liza dumerili

Liza dumerili

Liza falcipinnis

Liza falcipinnis

Liza grandisquamis

Liza saliens

Liza ramado

Liza aurata

Liza aurata

Chelon labrosus

Liza ramado

Liza ramado

Mugil bananensis

Mugil bananensis

Mugil bananensis

Mugil cephalus

Mugil cephalus

Mugil ashanteensis

Mugil ashanteensis

Mugil ashanteensis

Mugil ashanteensis

Mugil ashanteensis

Mugil metzelaai

Mugil metzelaa

Mugil capurn

Mugil capurrii

Oedalechilus labeo

Mugil cephalus

Mugil cephalus
Mugil sp. I

ugil sp. I

ugil sp. I

gil sp. I

mugil sp.

ugil sp.

mili sp. 1

ming.1

(Mpil sp.

agit sp.

Mugil sp.

agil sp.

Mugil sp.1

Mugil sp. 1

Mugil sp. I

Mugil sp. L

Mugil sp. L

Mugil sp. $\mathrm{L}$

Mugil sp. L

Mugil sp. 0

Mugiliza

ugil liza

Mugil sp. $\mathrm{H}$

Planiliza haematocheila Chelon bandialensis Chelon bandialensis Chelon dumerili Chelon dumerif

olcocipins

Neochelon falcipinnis

Pachon falipints

Parachelon grandisquamis $M$ Parachelon grandisquamis $M$ Chelon saliens

Chelon ramado

Chelon auratus

Chelon auratus

Chelon labrosus

Chelon labrosus

Chelon ramado

Chelon ramado

Mul bananensis

Mugl bananensis

Mugil cephalus

Mugil cephalus

Mugil sp. J

Mugil sp. $\mathrm{J}$

Mugil sp.

Mugil sp. $\mathrm{J}$

Mugil sp. J

Mugil sp. M

Mugil sp. M

Mugil capurrii

Mugil capurriil

Oedalechilus labeo

Mugil sp. B

Mugil sp. B 


\begin{tabular}{|c|c|}
\hline HQ024980 & \\
\hline HQ131880 & Agmo \\
\hline HQ131881 & Jopi \\
\hline HQ131882 & 44La \\
\hline HQ131883 & 10Ldu \\
\hline HQ131884 & 92NWAMce \\
\hline HQ131885 & 93NWAMce \\
\hline HQ131886 & 107Mcu \\
\hline HQ131887 & 108Mcu \\
\hline HQ131888 & WAMcu \\
\hline HQ131889 & Mho \\
\hline HQ131890 & Minc1 \\
\hline HQ131891 & Minc2 \\
\hline HQ131892 & 417-Mc-SMNH3 \\
\hline HQ149082 & YM17 \\
\hline HQ149083 & YM10 \\
\hline HQ149710 & 347 \\
\hline HQ149711 & 344 \\
\hline HQ149712 & 295 \\
\hline HQ149713 & 298 \\
\hline HQ149885 & - \\
\hline HQ149965 & - \\
\hline HQ285928 & - \\
\hline HQ285929 & - \\
\hline HQ285930 & - \\
\hline HQ654717 & - \\
\hline HQ654718 & - \\
\hline HQ654719 & - \\
\hline HQ654720 & - \\
\hline HQ654721 & - \\
\hline HQ682701 & - \\
\hline HQ682702 & - \\
\hline HQ682703 & - \\
\hline HQ682704 & - \\
\hline JF493283 & - \\
\hline JF493284 & - \\
\hline JF493796 & - \\
\hline JF493797 & - \\
\hline JF493798 & - \\
\hline JF493799 & - \\
\hline JF493800 & - \\
\hline JF493801 & - \\
\hline JF493802 & - \\
\hline JF493803 & - \\
\hline JF493804 & - \\
\hline JF493805 & - \\
\hline JF493806 & - \\
\hline JF493807 & - \\
\hline JF493808 & - \\
\hline JF493809 & - \\
\hline JF493810 & - \\
\hline JF493811 & - \\
\hline JF493812 & - \\
\hline JF493813 & - \\
\hline JF493905 & - \\
\hline JF493906 & - \\
\hline JF493907 & - \\
\hline JF493950 & - \\
\hline JF493952 & - \\
\hline JF494774 & - \\
\hline JF494775 & - \\
\hline JF494776 & - \\
\hline
\end{tabular}

FDA 1-33

$\cdot$

$\cdot$

\begin{tabular}{ll}
- & West Africa \\
- & Belize \\
- & Kourou, French Guiana \\
- & Kourou, French Guiana \\
- & Arroyo Zanja, Uruguay \\
- & Japan \\
- & Japan \\
\hline & Charleston, South Carolina, USA \\
UDOV-89 & Indian River, Florida, USA \\
Kourou, French Guyana \\
La Restiga, Margarita Island, Venezuela
\end{tabular}

NPPF1155

NPPF1167

ECAM780

ECAM782
ECAM785

Lmel4

(1)

Lmel2

sub1

Lsub4-LdB

Lsub3-LdB

Lsub2-LdB

ADC2221-2

DC222.1-1

ADC222.2-3

DC222.2-1

ADC222.3-1

ADC222.3-3

ADC08222.5

Smith222.6\#1

Smith222.6\#2

Smith222.4\#3.1

Smith222.4\#4.1

Smith222.4\#1.1

Smith222.4\#2.

ADC222.7-1

Smith222.7\#5.1

Smith222.7\#3.1

ADC222.7-2

ADC222.8-1

ADC222.10-2

ADC222.10-1

-1
-10

ADC222.12-

ADC222.12-1
Market sample, Washington DC, USA

Caribbean Sea

Changuinola River, Boca del Toro, Panama

Dakhla, Morocco

issau, Bissau Guine

adre, Mexico

sland, Honduras

layband National Park, Bushehr, Iran $\left(27.40^{\circ} \mathrm{N} 52.60^{\circ} \mathrm{E}\right)$

Nayband National Park, Bushehr, Iran $\left(27.40^{\circ} \mathrm{N} 52.60^{\circ} \mathrm{E}\right)$

La Restinga Lagoon, Venezuela

La Restinga Lagoon, Venezuela

Talisay, Taal Lake, Calabrazon, Philipinnes

Talisay, Taal Lake, Calabrazon, Philipinnes

Talisay, Taal Lake, Calabrazon, Philipinnes

Talisay, Taal Lake, Calabrazon, Philipinnes

Talisay, Taal Lake, Calabrazon, Philipinnes

Aplaya, Laguna de Bay, Calabarzon, Philippines

Aplaya, Laguna de Bay, Calabarzon, P ilippines

Aplaya, Laguna de Bay, Calabarzon, Philippines

Aplaya, Laguna de Bay, Calabarzon, Philippin

Kosi Lakes, KwaZulu-Natal, South Africa

Sezela Estuary, KwaZulu-Natal, South Africa

Sezela Estuary, KwaZulu-Natal, South Africa

Sezela Estuary, KwaZulu-Natal, South Africa

Mhlangary, Kwazulu-Natal, Soun Arica

Mhlanga Estuary, KwaZulu-Natal, South Africa

Mhlanga Estuary, KwaZulu-Natatal, South Africa

Pomene, Mozambique

Mlalazi Estuary, KwaZulu-Natal, South Africa

Mlalazi Estuary, KwaZulu-Natal, South Africa

St Lucia, South Africa

StLucia, South Afica

St Lucia, South Afica

Danger Point, Western Cape, South Africa

Table Bay, South Africa

Danger Point Western Cape, South Africa

Inyoni Rocks, KwaZulu-Natal, South Africa

Mhlanga Estuary, KwaZulu-Natal, South Africa

Mhlanga Estuary, KwaZulu-Natal, South Africa

Mhlanga Estuary, KwaZulu-Natal, South Africa

Mbezane Estuary, KwaZulu-Natal, South Africa

Mbezane Estuary, KwaZulu-Natal, South Africa

Richard's Bay, KwaZulu-Natal, South Africa

Mpenjati Estuary, KwaZulu-Natal, South Africa
M. Nirchio

A. Pariselle

J.-D. Durand

F. Garcia de Leon

Kreiser

Belize Workshop participants

R. Vigouroux

21 Feb. $2007 \quad$ S. Kullander

01 June 2005 R. Paperno

13 Jan. 2007 R. Vigouroux

15 Dec. $2009 \quad$ H. Asgharian

5 Dec. $2009 \quad$ H. Asgharian

M. Nirchio

M. Nirchio

14 Aug. $2010 \quad$ J. Quilang

14 Aug. 2010 J. Quilang

14 Aug. 2010 J. Quilang

14 Aug. 2010 J.Qulang

14 Aug. 2010 J. Quilang

J. Quilang

J. Quilang

J. Quilang

19 May 2004 J. Quilang

19 May $2004 \quad$ S. Weerts

04 Dec. 2003 A.D. Connell

04 Dec. 2003 A.D. Connell

04 Dec. 2003 A.D. Connell

13 Aug. 2003 A.D. Connel

13 Aug. 2003 A.D. Connell

13 Aug. 2003 A.D. Connell

01 May 2008 A.D. Connel

20 Sep. 2005 A.D. Connell

20 Sep. 2005 A.D. Connell

11 Feb. 2006 A.D. Connell

12 Feb. 2006 A.D. Connell

13 Feb. 2006 A.D. Connell

14 Feb. 2006 A.D. Connell

14 Oct. $2004 \quad$ A. Bok

01 Oct. $2004 \quad$ G. Beukes

01 Oct. $2004 \quad$ G. Beukes

01 Oct. $2004 \quad$ A. Bok

03 June 2004 A.D. Connell

19 Aug. 2004 A.D. Connell

19 Aug. 2004 A.D. Connell

9 Aug. 2004 A.D. Connell

13 Jan. 2005 A.D. Connell

13 Jan. 2005 A.D. Connell

21 Oct. 2003 A.D. Connell

14 Jan. 2005 A.D. Connell

13 Aug. 2003 A.D. Connel
Barcoding

Mugil cephalus Mugil sp. B

Phylogeny Liza aurata

Phylogeny Liza dumerili Chelon dumerill

Phylogeny - Mugil cophalus

mugil ephalus

Phylogeny Mugil curema

Phylogeny Mugil curema

Phylogeny Mugil hospes

Phylogeny Mugilincilis

Phylogeny Mugil incilis

Phy

Phlogegraphy Mulcephals

Phylogeny

Phylogeny Muilcempals

Phylogeny Mugil liza

Phylogeny Mugil liza

Barcoding Mugilidae sp.

Barcoding

Barcoding

Barcoding

Barcoding

Barcoding

Barcoding

Barcoding

Barcoding

Barcoding

Barcoding

Barcoding

Barcoding

Barcoding

Barcoding

Barcoding

Barcoding

Barcoding

Barcoding

Barcoding

Barcoding

Barcoding

Barcoding

Barcoding

Barcoding

Barcoding

Barcoding

Barcodin

Barcoding

Barcoding

Barcoding

Barcoding

Barcoding

Barcodin

Barcoding

Barcoding

Barcoding

Barcoding

Barcoding

Barcoding
Valamugil buchanan

Mugil incills

Mugil incilis

Liza melinoptera

Liza melinoptera

Liza melinoptera

Liza subviridis

Liza subviridis

Liza subviridis

Liza subviridis

Crenimugil crenilabis

Liza alata

Liza alat

Liza dumerili

Liza dumerili

Liza mameril

Liza melinoptera

Liza melinoptera

Liza melinoptera

Liza melinoplera

Liza melinoptera

Liza richardsoni

Liza richardsoni

Liza richardsoni

Liza richardsonir

Liza tricuspidens

Mugilcephalus

Mugil cephalus

Myxus capensis

Myxus capensis

Valamugil buchanani

Valamugil cunnesius
Mugil sp. B
Mugil sp. B

Mugil curema

Mugil curema

Mugil sp. M

Muil sp. R

Muilincilis

Mugil curema

Mugil sp. C

ugil sp. C

Mugil sp. B

Mugil liza

Muniza

Cenimugil sp. B

Mugil incilis

Mugil incilis

Mugil incilis

Planiliza sp. $\mathrm{G}$

Planiliza sp. G

Planiliza sp. $G$

Planiliza sp. G

Planiliza subviridis

Planiliza subviridis

Planiza subvirdis

Crenimugil crenilabis

Crenimugil crenilabis

Planiliza alata

Planiliza

Chniza ala

Chelon sp. B

Chelon sp. B

Chelon sp. B

Planiliza melinoptera

Chelon sp. A

Planiliza melinoptera

Planiza melinoplera

Planiliza melinoptera

Planilza mellioptera

Chelon richardsoni

Chelon nichardsonit

Chelon richardsoni

Chelon richardsomi

Mugil sp. E

Mugl sp.

Pseudomyxus capensis

Pseudomyxus capen

Crenimugil sp. A

Osteomugil sp. B 


$\begin{array}{llll}\text { JF494777 } & - & \text { ADC222.14-1 } & \text { Mpenjati Estuary, KwaZulu-Natal, South Africa } \\ \text { JF494780 } & - & \text { ADC222.15-2 } & \text { Mbezane Estuary, KwaZullu-Natal, South Africa } \\ \text { JF494781 } & - & \text { ADC222.15-1 } & \text { Mhlanga Estuary, KwaZulu-Natal, South Africa } \\ \text { JF952795 } & & - & \text { Arasaki, Yokosuka, Kanto, Japan (35.00 }{ }^{\circ} \text { N 139.50 } \\ \text { JF5) } \\ \text { JF52796 }\end{array}$

\begin{tabular}{|c|c|}
\hline 13 Jan. 2005 & A.D. Connell \\
\hline 14 Jan. 2005 & A.D. Connell \\
\hline 13 Aug. 2003 & A.D. Connell \\
\hline 13 Sep. 2005 & S. Chow \\
\hline 13 Sep. 2005 & S. Chow \\
\hline 12 Oct. 2010 & J. Quilang, J. Tango \\
\hline 12 Oct. 2010 & J. Quilang, J. Tango \\
\hline Jan. 2011 & V. Sachithanandam et al \\
\hline 04 June 2011 & J. Zhang \\
\hline 09 June 2011 & J. Zhang \\
\hline 12 Apr. 2011 & J. Zhang \\
\hline 04 June 2011 & J. Zhang \\
\hline 12 Apr. 2011 & J. Zhang \\
\hline 12 Apr. 2011 & J. Zhang \\
\hline 12 Apr. 2011 & J. Zhang \\
\hline 26 Feb. 2011 & J. Zhang \\
\hline 26 Feb. 2011 & J. Zhang \\
\hline 09 June 2011 & J. Zhang \\
\hline 18 May 2011 & J. Zhang \\
\hline 18 Apr. 2011 & J. Zhang \\
\hline 12 Apr. 2011 & J. Zhang \\
\hline & Z. Wu, F. You \\
\hline . & M. Ashiq Ur Rahman \\
\hline . & M. Ashiq Ur Rahman \\
\hline . & M. Ashiq Ur Rahman \\
\hline . & M. Ashiq Ur Rahman \\
\hline - & M. Ashiq Ur Rahman \\
\hline & M. Ashiq Ur Rahman \\
\hline . & M. Ashiq Ur Rahman \\
\hline . & M. Ashiq Ur Rahman \\
\hline . & M. Ashiq Ur Rahman \\
\hline & M. Ashiq Ur Rahman \\
\hline 03 Nov. 2005 & P. Keith, G. Marquet \\
\hline 13 Sep. 2008 & S. Sánchez et al. \\
\hline 22 Feb. 2006 & S. Kullander \\
\hline 25 Feb. 2006 & S. Kullander \\
\hline 15 Apr. 2005 & M. Nirchio \\
\hline Jan.-Apr. 2008 & M. Louis \\
\hline 09 May 2007 & M. Nirchio \\
\hline- & M. Nirchio \\
\hline 30 June 2005 & W.A. Matamoros \\
\hline 13 Sep. 2008 & S. Sánchez et al. \\
\hline 10 Oct. 2006 & $\begin{array}{l}\text { J. Boubee } \\
\text { lat }\end{array}$ \\
\hline 24 May 2007 & C. Pöllabauer \\
\hline 01 June 2008 & B.W. Jamandre \\
\hline 18 Apr. 2007 & A. Ramirez \\
\hline 01 May 2010 & F.J. Garcia de Leon \\
\hline 01 May 2010 & F.J. Garcia de Leon \\
\hline 22 Dec. 2005 & H. Blel \\
\hline - & A. Pariselle \\
\hline Mar. 2010 & A. Pariselle \\
\hline 11 Aug. 2007 & P. Borsa \\
\hline 25 Aug. 2007 & P. Borsa \\
\hline 08 Mar. 2007 & P. Borsa \\
\hline 01 Apr. 2007 & P. Borsa \\
\hline 01 Apr. 2007 & P. Borsa \\
\hline 16 Nov. 2006 & K. Shibukawa \\
\hline 15 June 2008 & K.-N. Shen \\
\hline 19 Sep. 2008 & P. Borsa \\
\hline 28 June 2008 & P. Borsa, S. Andréfouët \\
\hline May 2004 & P. Renzo \\
\hline 19 Apr. 2005 & P.C. Heemstra et al. \\
\hline
\end{tabular}

Zhang and Hanner 2012 Barcoding Zhang and Hanner 2012 Barcoding Zhang and Hanner 2012 Barcoding Zhang and Her 2012 Barcoding Zhang 2012 Barcodilg Zhang and Hor 2012 Barcoding Zhang and Hanner 2012 Barcoding Zhang and Hanner 2012 Barcoding Zhang and Hanner 2012 Barcoding Zhang and Hanner 2012 Barcoding Zhang and Hanner 2012 Barcoding Zhang and Hanner 2012 Barcoding Zhang and Hanner 2012

Ashiq Ur Rahman et al. 2013 Barcoding Ashiq Ur Rahman et al. 2013 Barcoding Ashiq Ur Rahman et al. 2013 Barcoding Ashiq Ur Rahman et al. 2013 Barcoding Ashiq Ur Ranman elal. 2013 Barcoding Ashiq Ur Rahman et al. 2013 Barcoding Ashiq Ur Rahman et al. 2013 Barcoding Ashiq Ur Rahman et al. 2013 Barcoding Ashiq Ur Rahman et al. 20133 Barcoding Ashiq Ur Rahman et al. 2013 Barcoding Durand et al. 2012b Durand et al. 2012b Durand et al. 2012 Durand et al. 2012 Durand et al. 2012 Durand et al. 2012 Durand et al. 2012 Durand et al. 2012 Durand et al. 2012 Durand et al. 2012b Durand et al. 2012 Durand et al. 2012 Durand et al. 2012 Durand et al. 2012 Durand et al. 2012 Durand et al. 2012 Durand et al. 2012 Durand et al. 2012 Durand et al. 2012 Durand et al. 2012 Durand et al. 2012 Durand et al. 2012 Durand et al. 2012 Durand et al. 2012b Durand et al. 2012 Durand et al. 2012 Durand et al. 2012 Durand et al. 2012 Durand et al. $2012 b$ Durand et al. 2012b
Barcoding

Barcoding

Barcoding

Barcoding

Barcoding

Barcoding

Barcoding

Barcoding

Valamugil robustus Valamugil sehe Mugil cephalus Mugil cephalus

Liza macrolepis Liza macrolepis Liza macrolepis Mugil cephalus Mugil cephalus units ungil cephas Mugil ephalus Mugil cephalus Mugl cephalus Liza haematochella Liza haematocheila Liza haematochella Liza haematochella Liza haematochella Liza tade

Moolgarda cunnesius Valamugil speigleri Liza parsia

Ellochelon vaigiensis Moolgarda seheli Chelon subviridis Mugil cephalus Chelon planiceps Phylogeny
Phylogeny Phylogeny Phylogeny Phylogeny Phylogeny Phylogeny Phylogeny Phylogeny Phylogeny Phylogen Phylogeny Phylogeny Phylogeny Phylogeny Phylogeny Phylogeny Phylogeny Phylogeny Phylogeny Phylogeny Phylogeny Phylogeny Phylogeny Phylogeny Phylogeny Phylogeny Phylogeny Phylogeny Phylogeny

Chelon labrosus

Chelon labrosus

Chelon macrolepis

Chelon macrolepis

Chelon macrolepis

Chelon macrolepis

Chelon macrolepis

Chelon macrolepis

Chelon macrolepis

Chelon macrolepis

Chelon macrolepis

Chelon macrolepis
Osteomugil robustus Osteomugil sp. B Osteomugil sp. B Seriola sp.

Planiliza sp. $H$

Planiliza sp. $H$
Planiliza sp. $H$

Planiliza subviridis

Mugil sp. C

Mugil sp. C

Mugil sp. C

Mugil sp.C

Mugil sp.c

Mugil sp.c

Planiliza haematocheil

Planiliza haematocheila

Planiliza haematocheila

Planiliza haemalochelia

Planiliza haematocheila

Planiliza haematocheila Osteomugil sp.

Osteomugil cunnesius
Osteomugil sp. Osteomugil sp.

Planiliza subviridis Crenimugil sp. C

Planiliza subviridis

Mugil sp. B

Planiliza sp.

Agonostomus catalai Agonostomus catalai

Agonostomus monticola Dajaus sp. A

Agonostomus monticola Dajaus sp. A

Agonostomus monticola Dajaus sp. A

Agonostomus monticola Dajaus sp. A

Agonostomus monticola Dajaus sp. B

Agonostomus monticola Dajaus sp. B

Agonostomus monticola Dajaus sp. B

Agonostomus monticola Dajaus sp. B

Agonostomus monticola Dajaus sp. B

Aldrichetta forsteri Aldrichetta forsteri

Cestraeus oxyrhyncus Cestraeus oxyrhyncus

Cestraeus goldiei Cestraeus goldiei

Chaenomugil proboscideus Chaenomugil proboscideus

Chaenomugil proboscideus Chaenomugil proboscideus

Chaenomugil proboscideus Chaenomugil proboscideus Chelon labrosus
Planiliza sp. H

Planiliza sp. $\mathrm{H}$

Planiliza sp. H

Planiliza sp. $\mathrm{H}$

Planiliza sp. $\mathrm{H}$

Planiliza sp. $\mathrm{H}$

Planiliza sp. $\mathrm{H}$

Planiliza sp. H

Planiliza macrolepis
Planiliza macrolepis 


\begin{tabular}{|c|c|c|c|}
\hline JQ060422 & 144 & MNHN ICOS-00270 & Ra's al-Hadd, Oman \\
\hline JQ060423 & 145 & MNHN 2010-0027 & Ra's al-Hadd, Oman \\
\hline JQ060413 & 154 & - & Negombo lagoon, Sri Lanka \\
\hline JQ060427 & 109 & . & Viti Levu, Fiji \\
\hline JQ060428 & 111 & . & Viti Levu, Fiji \\
\hline JQ060430 & 175 & NRM-58400 & Kyaiko market, Myanmar \\
\hline JQ060431 & 176 & NRM-58557 & Kyaiko market, Myanmar \\
\hline JQ060429 & 122 & NTM S.15537-001 & Middle part beach, West Alligator Head, NT, Australia \\
\hline JQ060433 & 069 & - & Segara Amahan lagoon, Java, Indonesia \\
\hline JQ060434 & 070 & . & Negombo lagoon, Sri Lanka \\
\hline JQ060432 & 064 & NMMBP10796 & Cigu, Taiwan \\
\hline JQ060435 & 185 & MNHN 2009-0808 & Moruroa atoll, French Polynesia \\
\hline JQ060436 & 187 & - & Adhoo atoll, Maldives \\
\hline JQ060437 & 190 & MNHN 2008-1002 & Moorea, Iles du Vent French Polynesia \\
\hline JQ060438 & 191 & - & Tahiti, lles du Vent French Polynesia \\
\hline JQ060439 & 192 & MNHN 2009-1680 & Tuamotu, French Polynesia \\
\hline JQ060440 & 193 & SAIAB-86172 & Saipan, Mariana Islands \\
\hline JQ060441 & 194 & - & Loop Is., Chesterfield islands, Coral Sea \\
\hline JO060442 & 195 & SAIAB-77948 & Port Launay (SE beach). Seychelles \\
\hline JQ060443 & 046 & CSIRO-H4307-01 & Unspecified location, Australia \\
\hline JQ060444 & 051 & - & Batanta Island, West Papua \\
\hline JQ060445 & 053 & . & Tahiti, French Polynesia \\
\hline JQ060446 & 022 & STRI 21850 & Changuinola River, Boca del Toro, Panama \\
\hline JQ060447 & 061 & - & Tigris River, Turkey \\
\hline JQ060448 & 057x & NMMBP11540 & Yunlin, Taiwan \\
\hline JQ060451 & 165 & SAIAB-74457 & Sezela estuary, South Africa \\
\hline JQ060452 & $165 \mathrm{~b}$ & - & East K'monde estuary, South Africa \\
\hline JQ060449 & 162 & NTM S.16482-001 & Charlie's Creek, Daly River NT, Australia \\
\hline JQ060450 & 164 & NTM S.16483-001 & Near Crocodile Creek, Daly River NT, Australia \\
\hline JQ060453 & 037 & CSIRO-H4307-04 & unknown location, Australia \\
\hline JQ060454 & 039 & AMS I-43204-011 & Port Hacking, NSW, Australia \\
\hline JO060455 & 041 & - & Brisbane, Australia \\
\hline JQ060456 & 168 & - & Goulette, Tunisia \\
\hline JQ060457 & 169 & . & Agadir, Morocco \\
\hline JQ060458 & $171 \mathrm{~b}$ & . & Dakhla, Western Sahara \\
\hline JQ060459 & $171 \mathrm{c}$ & . & Khnifiss, Morocco \\
\hline JO060460 & $171 d$ & - & Khnifiss, Morocco \\
\hline JQ060461 & 054 & MNHN 2009-0790 & Saloum estuary, Senegal \\
\hline JQ060462 & 056 & - & Bubaque, Bijagos IIIds, Bissau Guinea \\
\hline JQ060463 & 073 & SAIAB-83185 & Toubacouta, Saloum estuary, Senegal \\
\hline JQ060464 & 076 & - & St Louis, Senegal \\
\hline JQ060465 & 077 & - & Fish market, Lome, Togo \\
\hline JQ060466 & 078b & - & Fish market, Bissau, Bissau Guinea \\
\hline JQ060467 & 079 & - & Great Fish River, South Africa \\
\hline JQ060468 & 080 & - & Great Fish River, South Africa \\
\hline JQ060469 & 083 & MNHN 2009-0730 & Toubacouta, Saloum estuary, Senegal \\
\hline JQ060470 & 084 & - & St Louis, Senegal \\
\hline JQ060471 & 085 & . & St Louis, Senegal \\
\hline JQ060472 & 086 & - & Fish market, Lome, Togo \\
\hline JQ060473 & 088 & - & Saloum, Sénégal \\
\hline JQ060474 & 089b & - & Fish market, Bissau, Bissau Guinea \\
\hline JQ060475 & 090 & MNHN 2009-731 & Saloum, Sénégal \\
\hline JQ060476 & 091 & SAIAB-83182 & Saloum, Sénégal \\
\hline JQ060477 & 095 & - & Yunlin, Taiwan \\
\hline JQ060478 & 102 & NMMBP11541 & Yunlin, Taiwan \\
\hline JQ060479 & 173 & - & Goulette, Tunisia \\
\hline JQ060480 & $174 b$ & - & Zerga, Morocco \\
\hline JQ060481 & 157 & SAIAB-64270 & Orange River, Namibia \\
\hline JQ060482 & 158 & - & Great Fish River, South Africa \\
\hline JQ060483 & 166 & . & Goulette, Tunisia \\
\hline JO060492 & 161 & SAIAB-78131 & Saint Lucia, South Africa \\
\hline JQ060490 & 113 & - & Al Khobar, Saudia Arabia, Persian Gulf \\
\hline
\end{tabular}

\begin{tabular}{|c|c|c|}
\hline 22 Mar. 2009 & P. Béarez & Durand et al. 2012b \\
\hline 22 Mar. 2009 & P. Béarez & Durand et al. 2012b \\
\hline 2007 & H. Ashanti & Durand et al. 2012b \\
\hline 19 Sep. 2008 & P. Borsa & Durand et al. 2012b \\
\hline 19 Sep. 2008 & P. Borsa & Durand et al. 2012b \\
\hline 15 Mar. 2008 & F. Fang, Thein Win & Durand et al. 2012b \\
\hline 15 Mar. 2008 & F. Fang, Thein Win & Durand et al. 2012b \\
\hline 09 June 2001 & H. Larson & Durand et al. 2012b \\
\hline July 2008 & S. Kleinertz, SPICE II project & Durand et al. 2012b \\
\hline 2007 & H. Ashanti & Durand et al. 2012b \\
\hline 13 Nov. 2008 & K.-N. Shen & Durand et al. 2012b \\
\hline June 2006 & P. Borsa et al. & Durand et al. 2012b \\
\hline 28 June 2008 & P. Borsa & Durand et al. 2012b \\
\hline 24 Mar. 2006 & Biocode project & Durand et al. 2012b \\
\hline Jul.-Aug. 2008 & A. Stein & Durand et al. 2012b \\
\hline Feb. 2009 & A. Stein & Durand et al. 2012b \\
\hline 24 May 2003 & K.A. Moots et al. & Durand et al. 2012b \\
\hline 22 Oct. 2008 & P. Borsa & Durand et al. 2012b \\
\hline 19 Apr. 2005 & P.C. Heemstra et al. & Durand et al. 2012b \\
\hline 31 July 1996 & G. Yearsley & Durand et al. 2012b \\
\hline 04 Dec. 2007 & P. Borsa & Durand et al. 2012b \\
\hline 01 Aug. 2008 & A. Stein & Durand et al. 2012b \\
\hline - & - & Durand et al. 2012b \\
\hline 27 Apr. 2005 & E. Ülü & Durand et al. 2012b \\
\hline 21 Feb. 2008 & K.-N. Shen & Durand et al. 2012b \\
\hline May 2005 & A.D. Connell & Durand et al. 2012b \\
\hline 02 Feb. 2010 & P. Cowley & Durand et al. 2012b \\
\hline 12 June 2007 & H. Larson & Durand et al. 2012b \\
\hline 13 June 2007 & H. Larson & Durand et al. 2012b \\
\hline 25 July 2005 & G. Yearsley via Raptis and Sons, Colmslie & \& Durand et al. 2012b \\
\hline July 1997 & B. Pease et al. & Durand et al. 2012b \\
\hline & W. Knibb & Durand et al. 2012b \\
\hline \multirow[t]{2}{*}{ Apr. 2003} & H. Blel & Durand et al. 2012b \\
\hline & A. Pariselle & Durand et al. 2012b \\
\hline Mar. 2010 & A. Pariselle & Durand et al. 2012b \\
\hline Mar. 2010 & A. Pariselle & Durand et al. 2012b \\
\hline Mar. 2010 & A. Pariselle & Durand et al. 2012b \\
\hline 2008 & O. Diouf & Durand et al. 2012b \\
\hline May 2008 & Y. Bettarel & Durand et al. 2012b \\
\hline 06 Mar. 2009 & O. Sadio, J. Raffray & Durand et al. 2012b \\
\hline 26 Jan. 2008 & J.-D. Durand & Durand et al. 2012b \\
\hline 19 June 2007 & J.F. Trape & Durand et al. 2012b \\
\hline 04 May 2010 & J.-D. Durand & Durand et al. 2012b \\
\hline 12 May 2005 & A.K. Whitfield, J.-D. Durand & Durand et al. 2012b \\
\hline 12 May 2005 & A.K. Whitfield, J.-D. Durand & Durand et al. 2012b \\
\hline 06 Mar. 2009 & O. Sadio & Durand et al. 2012b \\
\hline 26 Jan. 2008 & J.-D. Durand & Durand et al. 2012b \\
\hline 26 Jan. 2008 & J.-D. Durand & Durand et al. 2012b \\
\hline 19 June 2007 & J.F. Trape & Durand et al. 2012b \\
\hline 06 Mar. 2009 & O. Sadio et al. & Durand et al. 2012b \\
\hline 04 May 2010 & J.-D. Durand & Durand et al. 2012b \\
\hline 06 Mar. 2009 & O. Sadio & Durand et al. 2012b \\
\hline 06 Mar. 2009 & O. Sadio & Durand et al. 2012b \\
\hline 03 Dec. 2007 & K.-N. Shen & Durand et al. 2012b \\
\hline 03 Dec. 2007 & K.-N. Shen & Durand et al. 2012b \\
\hline Apr. 2003 & H. Blel & Durand et al. 2012b \\
\hline Mar. 2010 & A. Pariselle & Durand et al. 2012b \\
\hline 12 June 2001 & J. Koekemoer & Durand et al. 2012b \\
\hline 12 May 2005 & A.K. Whitfield, J.-D. Durand & Durand et al. 2012b \\
\hline Apr. 2003 & H. Blel & Durand et al. 2012b \\
\hline 14 May 2005 & A.K. Whitfield et al. & Durand et al. 2012b \\
\hline Nov.-Dec. 2008 & Z. Batang, P. Premlal & Durand et al. 2012b \\
\hline
\end{tabular}

Phylogeny

Phylogeny

Phylogeny

Phylogeny

Phylogeny

Phylogeny

Phygony

Phylogeny

Phylogeny

Phylogeny

Phylogeny

Phylogeny

Phylogeny

Phylogeny

Phylogeny

Phylogeny

Phylogeny

Phylogeny

Phylogeny

Phylogen

Phylogeny

Phylogeny

Phylogeny

Phylogeny

Phylogeny

Phylogeny

Phylogeny

Phylogeny

Phylogeny

Phylogeny

Phylogeny

Phylogeny

Phylogeny

Phylogeny

Phylogeny

Phylogeny

Phylogeny

Phylogeny

Phylogeny

Phylogen

Phylogeny

Phylogeny

Phylogeny

Phylogen

Phylogeny

Phylogeny

Phylogeny
Phylogeny

Phylogeny
Phylogeny

Phylogeny
Phylogeny

Phylogeny
Phylogeny

Phylogeny
Phylogeny

Phylogeny

Phylogeny

Phylogeny

Phylogeny

Phylogeny

Phylogeny

Phylogeny

Phylogeny
Phylogeny
Chelon macrolepis

lon macrolepis

(cher

Chelon planiceps

Chelon planiceps

Chelon planiceps

Chelon subviridis

Chelon subviridis

Crenimugil crenilabis

Crenimugil crenilabis

Crenimugil crenilabis

Crenimugil crenilabis

Crenimugil crenilabis

Crenimg

Crenimugil crenilabis

Ellochelon vaigiensis

Ellochelon vaigiensis

Joturus pichardi

Liza abu

Liza affinis

Liza alata

Liza alata
Liza alata

Liza alata

Liza argentea

Liza argentea

Liza aurata

Liza aurata

Liza aurata

Liza aurata

Liza aurata

Liza bandialensis

Liza bandialensis

Liza dumerili

Liza dumeril

Liza dumerili

Liza dumerili

Liza dumerili

Liza falcipinnis

Liza falcipinnis

Liza falcipinnis

Liza falcipinnis

Liza grandisquamis

Liza grandisquamis

Liza grandisquamis

Liza haematocheil

Liza haematocheila

Liza richardsonii

Liza richardson

Liza saliens

Liza sp.
Planiliza macrolepis aniliza macrolepis

laniliza sp. H

Paniliza melinoptera

Planiliza melinoptera

Planiliza sp. 1

Planiliza sp.I

Planiliza subviridis

Planiliza subviridis

Planiliza subviridis

Crenimugil crenilabis

Cenimugil crenilabis

Cenimugil crenilabis

Cenimugil crenilabis

enimugil crenilabis

nimugil crenilabis

nimugil crenilabis

(C)

Ellochelon sp. A

(

Joturus pichardi

Planiliza abu

Planilza afinis

Liza alata

Liza alata

Liza ordensis

Aldrichetta argentea

Aldrichetta argentea

chous

Chelon auratus

Chelon auratus

Chelon auratus

Chelon auratus

Chelon auratus

Chelon bandialensis

Chelon bandialensis

Chelon dumerili

Chelon dumeril

Chelon dumeril

Chelon dumeril

Chelon sp. B

Chelon sp. B

Neochelon falcipinnis

Neochelon falcipinnis

Neochelon falcipinnis

Neochelon falcipinnis

Parachelon grandisquamis

Parachelon grandisquamis

Parachelon grandisquamis

Planiliza haematocheila

Planiliza haematocheila

Chelon ramado

Chelon ramado

Chelon richardsonii

Chelon richardson

Chelon saliens

Chelon sp. A

Planiliza sp. A 
Al Khobar, Saudia Arabia, Persian Gulf Pangasinan, Philippines

Negombo lagoon, Srila

Ho Chi Minh City, Viet Nam

NMMBP1155

NMMBP1079

Cigu, Taiwa

Quang Yen, Viet Nam

Sunday estuary, South Africa

AMS-I-33460-016 Beach 1Km S of McGowans Beach, Kalumburu, Australia

ASIZP0061397

Beach 1 Rm $S$ of McGow

KU:KUI:32523

MNHN 2008-0692

Saipan, Mariana Islands

Tahiti, lles du Vent, French Polynesia

Taisi, Taiwan

Eydhafushi, Baa Atoll, Maldives

Viti Levu, Fiji

Viti Levu, Fij

SW Lagoon, New Caledonia

Aurora Province, Philippines

$\begin{array}{ll}\text { NMMBP10777 } & \text { Taisi, Taiwan } \\ \text { - } & \text { Batanta Island, West Papua }\end{array}$

Garapan Lagoon off Oceanside Bar, Saipan, Mariana Is.

Al Khobar, Saudi Arabia, Persian Gulf

Ternay Bay, Seychelles

SAIAB-77896 Stone Town, Zanzibar, Tanzania

MNHN 2009-0815 Noumea, New Caledonia

NTM S.16014-002 South Goulburn Island, NT, Australia

NTM S.15362-001 Fish River, Daly River area, NT, Australia

SAIAB-77947 Port Launay (SE beach), Seychelles

La RHN 2011-0097 Lanion

MNHN ICOS-00266 Ra's al-Hadd, Oman

Negombo lagoon, Sri Lanka

MNHN 2011-0096 La Reunion

MNHN 2009-0733 Saloum estuary, Senegal

Ebrie lagoon, Ivory Coas

St Louis, Senegal estuary, Sénéga

Dakhla, Western Sahara

MNHN 2009-0795 Lomé, Togo

Martigues, France

Goulette, Tunisia

Merja Zerga, Morocco

Agadir, Morocco

Khnifiss, Morocco

UDOV-290 Coast of Iquique, Chil

UDOV-291

Coast of Iquique, Chi

Laguna madre, Mexico

NMMBP11545

Kaoping estuary, Taiwan

Hawai

Great Fish river estuary, South Afric

stuary, South Africa

St Lucia, South Africa

St Lucia, South Africa

St Lucia, South Africa

SW Lagoon, New Caledonia

\begin{tabular}{|c|c|}
\hline Nov.Dec. 2008 & Z. Batang, P. Premlal \\
\hline 01 July 2008 & B. W. Jamandre \\
\hline 01 July 2008 & B. W. Jamandre \\
\hline 2007 & H. Ashanti \\
\hline 20 Mar. 2010 & W.-J. Chen \\
\hline 13 Nov. 2008 & K.-N. Shen \\
\hline 21 Mar. 2010 & W.-J. Chen \\
\hline 22 July 2002 & L.-C. Chaung \\
\hline 08 Aug. 2007 & A.K. Whitfield \\
\hline 12 May 2005 & A.K. Whitfield, J.-D. Durand \\
\hline 06 Sep. 1992 & J. Paxton \\
\hline 22 Mar. 2010 & W.-J. Chen \\
\hline 05 May 2002 & K.-T. Shao \\
\hline 24 May 2003 & K.A. Moots et. al. \\
\hline June-Oct. 2008 & T.K. Ogawa \\
\hline 15 Mar. 2006 & S. Planes, Campagne Biocode \\
\hline 01 Aug. 2008 & A. Stein \\
\hline 18 Aug. 2007 & P. Borsa \\
\hline 15 June 2008 & K.-N. Shen \\
\hline 28 June 2008 & P. Borsa, S. Andréfouët \\
\hline 19 Sep. 2008 & P. Borsa \\
\hline 19 Sep. 2008 & P. Borsa \\
\hline 08 Apr. 2007 & P. Borsa \\
\hline 01 July 2008 & B.W. Jamandre \\
\hline 15 June 2008 & K.-N. Shen \\
\hline 04 Dec. 2007 & P. Borsa \\
\hline 24 May 2003 & K.A. Moots et. al. \\
\hline Nov.-Dec. 2008 & Z. Batang, P. Premlal \\
\hline 14 May 2005 & P.C. Heemstra et. al. \\
\hline 28 May 2010 & J.-D. Durand \\
\hline 08 Mar. 2007 & P. Borsa \\
\hline 25 Aug. 2007 & P. Borsa \\
\hline 30 Nov. 2004 & R. Williams \\
\hline 06 Sep. 2001 & R. Williams \\
\hline 19 May 2005 & P.C. Heemstra et. al. \\
\hline Oct. 2008 & P. Durville, N. Hubert \\
\hline 21 Mar. 2009 & P. Béarez \\
\hline 2007 & H. Ashanti \\
\hline Oct. 2008 & P. Durville, N. Hubert \\
\hline 06 Mar. 2009 & O. Sadio, J. Raffray, L. Tito \\
\hline Aug. 2007 & B. Gourene \\
\hline 26 Jan. 2008 & J.-D. Durand \\
\hline June 2006 & H. Masski \\
\hline 19 June 2007 & J.F. Trape \\
\hline 02 Aug. 2007 & G. Lepra \\
\hline 22 Dec. 2005 & H. Blel \\
\hline 09 Feb. 2006 & H. Nouiri \\
\hline & A. Pariselle \\
\hline Mar. 2010 & A. Pariselle \\
\hline 23 May 2005 & M. Nirchio \\
\hline 23 May 2005 & M. Nirchio \\
\hline June 2006 & F. J. Garciade Leon \\
\hline 28 Dec. 2007 & K.-N. Shen \\
\hline June-Oct. 2008 & T. Ogawa \\
\hline June-Oct. 2008 & T. Ogawa \\
\hline Oct. 2008 & M.T. Craig \\
\hline 12 May 2005 & A.K. Whitfield, J.-D. Durand \\
\hline 12 May 2005 & A.K. Whitfield, J.-D. Durand \\
\hline May, Nov. 2006 & A.K. Whitfield \\
\hline May, Nov. 2006 & A.K. Whitfield \\
\hline May, Nov. 2006 & A.K. Whitfield \\
\hline & \\
\hline
\end{tabular}

Durand et al. 2012b

Durand et al. 2012b

Durand et al. 2012b

Durand et al. 2012

Durand et al. 2012

Durand et al. 2012

Durand et al. 2012

Durand et al. 2012

Durand et al. 2012

Durand et al. 2012

Durand et al. 2012

Durand et al. 2012

Durand et al. 2012

Durand et al. 2012

Durand et al. 2012

Durand et al. 2012

Durnd al. 2012

Durand et al $2012 b$

Durand et al. $2012 b$

Durand et al. $2012 b$

Durand et at 2012

Durand et al. 2012b

Durand et al. 2012b

Durand et al. 2012

Durand et al. $2012 b$

Durand et al. $2012 b$

Durand et al. $2012 b$

Durand et al. 2012

Durand et al. 2012

Durand et al. 2012

Durand et al. 2012

Durand et al. 2012

Durand et al. 2012b

Durand et al. 2012b

Durand et al. 2012b

Durand et al. 2012b

Durand et al. 2012b

Durand et al. 2012

Durand et al. 2012

Durand et al. 2012b

Durand et al. 2012

Durand et al. 2012

Durand et al. 2012b

Durand et al. 2012

Durand et al. 2012

Durand et al. 2012

Durand et al. 2012b

Durand et al. 2012

Durand et al. 2012

Durand et al. 2012

Durand et al. 2012b

Durand et al. 2012

Durand et al. 2012

Durand et al. 2012b

Durand et al. 2012

Durand et al. $2012 b$

Durand et al. 2012
Phylogeny

Phylogeny

Phylogeny

Phylogeny Lizasp.

Phylogeny - Liza tricuspidens

Phylogeny - Moolyada cun

Phylogeny Molgarda cunnesius

Phylogeny Moolgard cunnesius

Phylogeny Mogra

Pholony

Molgarda engel

Molona

Phylogeny Molgarda engeli

Phylogeny Molgarda perusi

Phylogeny Molgarda perusil

Phygeny Molgarda perri

Phy Molgarda senell

Phylogeny

Phylogeny Moolgarda seheli

Phylogny

Phylogeny Moolgarda sehell

Morarda sehel

Phylogeny Moolgarda sehell

Phylogeny Moolgarda sehell

Phylogeny Moolgarda sehell

Phylogeny Moolgarda sehell

Molgarda sehel

Phyolgarda sehell

Molgarda sehel

Molgarda sehi

Phylogeny Moolgarda sehell

Molgarda seh.

Phylogeny Moolgarda sp.

Phylogeny Moolgarda sp.

Molgarda sp.

Phylongananensis

Phylogeny Mugil cephalus

Phylogeny Mugil cephalus

Mugil cephalus

Mugil cephalus

Mugil cephalus

Phyony

Phylogeny

Phlogeny

Phylogeny

Phylogeny

Phylogeny

(P)

Phylogeny Mugil cephalus
Planiliza sp. A

Planiliza sp. C

Planiliza sp. C

Planiliza sp. D

Planiliza Sp. D

Planiliza sp. E

Planiliza sp.G

Chelon tricuspidens

Osteomugil sp. B

Osteomugil cunnesius

Osteomugil cunnesius

Osteomugil engeli

Steomugil engell

Osteomugil engeli

Oscominger

Ostemugil perusi

Crenimuil sp. A

Crenimugil sp. A

Crenimugil sp. A

Crenimugil sp. A

Crenimugil sp. A

Crenimugil sp. A

Crenimugil sp. A

Crenimugil sp. A

Crenimugil sp. A

Crenimugil sp. B

Crenimugil sp. B

Crenimugil sp. B

Crenimugil sp. B

Crenimugil sp. C

Crenimugil sp. C

Crenimugil sp. C

Crenimugil sp. A

Crenimugil sp. A

Crenimugil sp. A

Crenimugil sp. B

Crenimugil sp. B

Mughansis

Mugil capurrii

Mugil capurrii

Mugil capurii

Mugil cephalus

Mugil cephalus

Mugil cephalus

Mugil cephalus

Mugil cephalus

Mugil sp. A

Mugil sp. A

Mugil sp. B

Mugil sp. C

Mugil Sp. D

Mugil sp. D

Mugil sp. D

Mugil sp. E

Mugil sp. E

Mugil sp. E

Mugil sp. E

Mugil sp. E

Mugil sp. F 
Pateu Dam, New Zeland

Pateu Dam, New Zeland

La Paz, Mexico

Paredon, Chiapas, Mexico

Paredon, Chiapas, Mexico

Lima, Peru

Waikato river, New Zelan

AMS-1-43204-082 Port Hacking, Point Danger, Sydney, Australia

Port Hacking, Point Dang

NMMBP10790

Taisi, Taiwan

Pearl River, China

NSMT-P77767 Atake river, Kagoshima Prefecture, Kyushu, Japan Khnifiss, Morocco

Saloum estuary, Senegal

MNHN 2009-734 Hann Bay, Dakar, Seneg

Boran Dabon, Forecariah estuary, Guinea

Lomé, Togo

Nokoue Lake, Benin

Peel Harvey estuary, Australia

Kaoping estuary, Taiwan

NMMBP11553

Kaoping estuary, Taiwan

MNHN 2009-0819

New Caledonia

Koné, New Caledonia

Koń, New Cadonia

Koń, New Cadodonia

Viti Levu, Fiji

NRM-56494 Arroyo Zanja, Uruguay

Arroyo Zanja, Uruguay

USMI field no. WAM08 Close to Michaels Rock, Guanaja Island, Honduras Laguna Madre, Mexico

Laguna Madre, Mexco
Itapissuma, Brazil

Guadeloupe

UDOV-201
MNHN 2009-0732

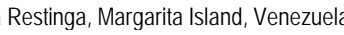

MNHN 2009-0732 Saloum estuary, Senegal

Nokoue, Lake, Benin

Nokoue, Lake, Benin

Lomé, Togo

SAIAB-83183 Saloum estuary, Senegal

Foundiougne, Saloum estuary, Seneg

UDOV-69 La Restinga, Margarita Island, Venezuela

UDOV-70

Restinga, Margarita Island, Venezuela

Boca del Rio, Margarita Island, Venezuel

UDOV-71

Boca del Rio, Margarita Island, Venezuela

La Paz, Mexico

Barra San José, Chapas, Mexico

NRM-53693

Santiago, El Salvador

Paredon, Chapas, Mexico

KU:KUl:40336 Mouth of Cerique estuary, El Salvador

UDOV-186

Chorreras Bay, Panama, Pacific coast

Chorreras Bay, Panama, Pacific coas

Barra San José, Chapas, Mexico

Barra San José, Chapas, Mexico

North Peru

Salango, Ecuado

KU:KUl:40290 Sand flats at mouth of Cerique Estuary, El Salvador

St. Johns college shore, Belize City, Belize
03 Nov. $2006 \quad$ J. Boubee

23 June $2006 \quad$ F.J. Garcia de Leon

Apr. 2010 P. Diaz Jaimes

21-24 June $2005 \mathrm{~S}$. Sirvas

28 June $1905 \quad J$. Boubee

17 Nov. $2005 \quad$ K. Rowling

Jull $1997 \quad$ B. Pease et

K.-N. Shen

May $2005 \quad$ X.P. Nie

04 July 2007 S. Chiba

Mar. 2010 A. Parisel

Dec. Mar. 2009 J.-D. Durand
J.

06 Mar. 2009 J.D. Durand

20 Feb. 2006 S.B. Camara

19 June 2007 J.F. Trape

12 Apr. 2007 P. Laleye

05 Jan. 2006 K. Smith

29 Dec. $2007 \quad$ K.-N. Shen

11 Aug. $2007 \quad$ P. Borsa

08 Apr. 2007 P. Borsa

11 Aug. 2007 P. Borsa

11 Aug. 2007 P. Borsa

19 Sep. 2008 P. Borsa

$21 \mathrm{Feb} .2007$ S. Kullander

$21 \mathrm{Feb} .2007$ S. Kullander

15 July 2008 W.A. Matamoro

July $2006 \quad$ F.J. Garcia de Leon

03 Dec. 2004 R. Lessa

30 June 1905 M. Louls

25 Jan. 2005 M. Nirchio

06 Mar. 2009 O. Sadio

12 Apr. 2007 P. Laleye

12 Apr. 2007 P. Laleye

19 June 2007 J.F. Trape

19 June 2007 J.F. Trap

06 Mar. 2009 O. Sadio

Dec. $2005 \quad$ O. Diouf

06 Apr. $2004 \quad$ M. Nirchio

06 Apr. $2004 \quad$ M. Nirchio

12 Apr. $2004 \quad$ M. Nirchio

10 Apr. 2004 M. Nirchio

23 June $2006 \quad$ F.J. Garcia de Leon

Apr. $2010 \quad$ N. Laurrabaquio Alvarado

25 Feb. $2006 \quad$ S. Kullander

09 Apr. $2010 \quad$ N. Laurrabaquio Alvarado

20 June 2008 A.C. Bentley, K. Lara

05 Dec. 1999 M. Nirchio

05 Dec. 1999 M. Nirchio

08 Apr. $2010 \quad$ N. Laurrabaquio Alvarado

08 Apr. 2010 N. Laurrabaquio Alvarado

S. Sirvas

11 Nov. 2008 P. Béarez

16 June 2008 A.C. Bentley, K. Lara

08 July 1999 Belize Workshop participants
Durand et al. 2012b

Durand et al. 2012b

Durand et al. 2012b

Durand et al. 2012b

Durand et al. 2012b

Durand et al. 2012

Durand et al. 2012

Durand et al. 2012b

Durand et al. 2012

Durand et al. 2012b

Durand et al. 2012

Durand et al. 2012

Durand et al. 2012b

Durand et al. 2012

Durand et al. 2012

Durand et al. $2012 b$

Durand et al. 2012

Durand et al. 2012

Durand et al. 2012

Durand et al. 2012

Durand et al. $2012 \mathrm{~b}$

Durnend 2012

Durand etal $2012 b$

Durand $2012 b$

Durnd 2012

Durand et al. 2012b

Durand et al. 2012b

Durand et al. 2012

Durand et al. 2012

Durand et al. 2012b

Durand et al. 2012

Durand et al. 2012b

Durand et al. 2012

Durand et al. 2012b

Durand et al. 2012

Durand et al. 2012

Durand et al. 2012

Durand et al. 2012

Durand et al. $2012 \mathrm{~b}$

Durand et al. 2012b

Durand et al. 2012

Durand et al. 2012b

Durand et al. 2012

Durand et al. 2012

Durand et al. 2012b

Durand et al. $2012 b$

Durand et al. 2012b

Durand et al. 2012

Durand et al. $2012 \mathrm{~b}$

Durand et al. 2012b

Durandetal. $2012 b$

Durand et al. 2012b

Durand elal 2012

Durand et al. 2012

Durand et al. 2012
Phylogeny

Mugil cephalus

Mugil cephalus

Mugil cephalus

Mugil cephalus

Phylogny

Phylogeny Mugil cephalus

Phylogeny Mugil cephalus

Phylogeny Mugil cephalus

Phylogeny Mugil cephalus

Phylogeny Mugil cephalus

Phylogeny Mugil cephalus

Phylogeny Mugil cephalus

Phylogeny Mugil cephalus

Phylogeny Mugil cephalus

Phylogeny Mugil cephalus

Phylogeny Mugil cephalus

Phylogeny Mugil cephalus

Phylogeny Mugil cephalus

Phylogeny Mugil cephalus

Phylogeny Mugil cephalus

Phylogeny Mugi cephalus

Phylogeny Mugil cephalus

Mugi cephalus

Phylogeny Mugic cephalus

Mugi cephalus

Mugin cephalus

Mugil cephalus

Mughrom

Phylogeny Mugic curema

Magil curema

Phylogeny Mugic curema

Phylogeny - Mugr curema

Phylogeny Mugil curema

Phylogeny Mulc curema

Phylogeny Mugic curema

Phylogeny Mugi curema

Phylogny Mugic curema

Phylogeny Mugil curema

Phylogny Mulcurema

Phylogny Mugicurema

Phylogny Mugi curema

Phylogny Mugi curema

Phylogny Mugil curema

Phylogny Mugil curema

Phylogny Mugi curema

Phylogeny Mugil curema

Phylogeny Mugil curema

Phylogeny Mugil curema

Phylogeny Mugil curema

Phylogeny Mugi curema

Phylogeny Mugil curema

Mugil sp. F

Mugil sp.

Mugil sp. $G$

Mugil sp. $G$

Mugil sp.

Mugil sp. $\mathrm{H}$

Mugil sp. $\mathrm{H}$

Mugil sp. $\mathrm{H}$

Mugil sp. I

Mugil sp.

Mugil sp. I

Mugil sp. J

Mugil sp. $\mathrm{J}$

Mugil sp. $\mathrm{J}$

Mugil sp. $\mathrm{J}$

Mugil sp. J

Mugil sp. J

Mugil sp. J

mail sp. $\mathrm{J}$

Mugil sp. K

Mugil sp. L

Mugil sp. $\mathrm{L}$

Mugil Sp. L

Mugil sp. L

Muil sp. $\mathrm{L}$

Mugil sp. L

Mul sp. L

Mugil sp.L

Mugil curema

Mugil curema

Mugil curema

Mugil curema

vugil curema

Mugicurema

Mugil curema

Mugil sp. M

Mugil sp. M

Mugil sp. M

Mugil sp.M

Mugl sp. M

Mugh sp.M

Mugil sp. M

Mugil sp.N

Mugl sp.N

Mugil sp. N

Mugil sp. N

Magil sp.

Mugil sp. 0 


\begin{tabular}{|c|c|c|c|}
\hline JQ060601 & 429 & KU:KUl:40290 & Sand flats at mouth of Cerique Estuary, El Salvador \\
\hline JQ060602 & 430 & - & Mangrove upstream Cerique estuary, El Salvador \\
\hline JQ060607 & 306 & . & West side of Turneffe Caye, deep hole, Belize \\
\hline JQ060608 & 302 & . & Kourou, French Guyana \\
\hline JQ060609 & 299 & - & Kourou, French Guyana \\
\hline JO060610 & 378 & NRM-56496 & Arrovo Zanja, Uruquay \\
\hline JQ060611 & 379 & NRM-55970 & Laguna del Diaro, Uruguay \\
\hline JQ060612 & 305 & UDOV-88 & Boca del Rio, Margarita Island, Venezuela \\
\hline JQ060613 & $305 \mathrm{~b}$ & UDOV-190 & Chorreras Bay, Pacific coast of Panama \\
\hline JQ060614 & 291 & UDOV-72 & Boca del Rio, Margarita Island, Venezuela \\
\hline JQ060615 & 019 & - & East Kleinemonde estuary, South Africa \\
\hline JQ060616 & 020 & NMV A 25183-002 & unknown location, Australia \\
\hline JQ060617 & 003 & SAIAB-86170 & Saipan, Mariana Islands \\
\hline JQ060618 & 003b & MNHN 2009-1681 & Arutua atoll, Tuamotu \\
\hline JQ060619 & 181 & - & Goulette, Tunisia \\
\hline JO060620 & 243 & NMMBP10775 & Orchid Island, Taiwan \\
\hline JQ060621 & 118 & & Segara Amahan lagoon, Java, Indonesia \\
\hline JQ060622 & 016 & NRM 59032 & Mandalay fish market, Myanmar \\
\hline JO060623 & 015 & NTM S.16483-002 & Near Crocodile Creek, Daly river, NT. Australia \\
\hline JQ060624 & 013 & FNHM 172496 & Dhaka, Bangladesh \\
\hline JQ060625 & 014 & NRM 58582 & Myoma market, Myanmar \\
\hline JQ060626 & 017 & AMS I-43255-002 & Williams River, Queensland, Australia \\
\hline JQ060627 & 245 & MNHN 2009-0816 & New Caledonia \\
\hline JQ060628 & 254 & SAIAB-78293 & Umgazana, South Africa \\
\hline JQ060641 & 248 & - & Kenya \\
\hline JQ060642 & 252 & - & Kenya \\
\hline JQ060629 & 259 & SAIAB-78094 & Nkadusweni estuary, South Africa \\
\hline JQ060630 & 260 & SAIAB-78137 & Poenskop, South Africa \\
\hline JQ060521 & 255 & MNHN 2011-0095 & Réunion Island \\
\hline J0060639 & 268 & - & Aurora Province, Philippines \\
\hline JQ060640 & 271 & - & Negombo lagoon, Sri Lanka \\
\hline JO060635 & 229 & - & Nha Trang City, Khanh Hoa Province, Viet Nam \\
\hline JQ060636 & 238 & - & Viti Levu, Fiji \\
\hline JQ060637 & 239 & - & Viti Levu, Fiji \\
\hline JQ060638 & 241 & ASIZP0064762 & Pingdon, Taiwan \\
\hline JQ343910 & - & IOCASFY-Lh-002 & Zhimai River, China \\
\hline JO365431 & . & HRCB:40559 & 23.863 S 46.152 W. Sao Paulo, Brazil \\
\hline JO365432 & . & HRCB:40566 & 23.863 S 46.152 W. Sao Paulo, Brazil \\
\hline JO365433 & - & HRCB:46936 & 23.863 S 46.152 W. Sao Paulo, Brazil \\
\hline JQ365434 & . & HRCB:46939 & 23.863 S 46.152 W, Sao Paulo, Brazil \\
\hline JQ365435 & . & HRCB:40558 & 23.863 S 46.152 W, Sao Paulo, Brazil \\
\hline JQ365436 & - & HRCB:40506 & 23.863 S 46.152 W, Sao Paulo, Brazil \\
\hline JQ365437 & - & HRCB:46938 & 23.863 S 46.152 W, Sao Paulo, Brazil \\
\hline JQ365438 & - & HRCB:40589 & 23.863 S 46.152 W, Sao Paulo, Brazil \\
\hline JQ365439 & - & HRCB:40560 & 23.863 S 46.152 W, Sao Paulo, Brazil \\
\hline JQ365440 & - & HRCB:40557 & 23.863 S 46.152 W, Sao Paulo, Brazil \\
\hline JQ365441 & . & HRCB:46935 & 23.863 S 46.152 W, Sao Paulo, Brazil \\
\hline JQ365442 & . & HRCB:46937 & 23.863 S 46.152 W, Sao Paulo, Brazil \\
\hline JQ365443 & - & HRCB:51316 & 25.105 S 47.781 W, Sao Paulo, Brazil \\
\hline JQ365444 & - & HRCB:51320 & 25.105 S 47.781 W, Sao Paulo, Brazil \\
\hline JQ365445 & - & HRCB:51319 & 25.105 S 47.781 W, Sao Paulo, Brazil \\
\hline JQ365446 & - & HRCB:51315 & 25.105 S 47.781 W, Sao Paulo, Brazil \\
\hline JQ365447 & . & HRCB:40546 & 23.863 S 46.152 W, Sao Paulo, Brazil \\
\hline JQ365448 & . & HRCB:46874 & 24.616 S 46.133 W, Sao Paulo, Brazil \\
\hline JQ365449 & . & HRCB:35140 & 23.506 S 45.034 W, Sao Paulo, Brazil \\
\hline JQ431670 & - & MBIO1645.4 & Moorea, Society Islands \\
\hline JQ431701 & - & MBIO810.4 & Haapiti, Moorea, Society Islands \\
\hline JQ431702 & . & MBIO809.4 & Haapiti, Moorea, Society Islands \\
\hline JQ431911 & . & MBIO814.4 & Haapiti, Moorea, Society Islands \\
\hline JQ431912 & . & MBIO813.4 & Haapiti, Moorea, Society Islands \\
\hline JO431913 & - & MBIO 828.4 & Haapiti, Moorea, Society Islands \\
\hline JQ623946 & & & Turkey \\
\hline
\end{tabular}

\begin{tabular}{|c|c|}
\hline 16 June 2008 & A.C. Bentley, K. Lara \\
\hline 16 June 2008 & A.C. Bentley, K. Lara \\
\hline 08 July 1999 & Belize Workshop \\
\hline 12 Jan. 2007 & R. Vigouroux \\
\hline 12 Jan. 2007 & R. Vigouroux \\
\hline 21 Feb. 2007 & S. Kullander \\
\hline 21 Feb. 2007 & S. Kullander \\
\hline 01 June 2004 & M. Nirchio \\
\hline 05 Dec. 1999 & M. Nirchio \\
\hline 10 Apr. 2004 & M. Nirchio \\
\hline 21 Sep. 2007 & A.K. Whitfield \\
\hline 29 July 2003 & Sydney Wholesale Fish Ma \\
\hline 24 May 2003 & K.A. Moots et. al. \\
\hline Nov. 2009 & A. Stein \\
\hline Apr. 2003 & H. Blel \\
\hline 01 Jan. 2009 & K.-N. Shen \\
\hline July 2008 & S. Kleinertz, SPICE II project \\
\hline 26 Mar. 2008 & S. Kullander, T.-Y. Liao \\
\hline 13 June 2007 & H. Larson \\
\hline 13 Dec. 2008 & R.L. Mayden et al. \\
\hline 18 Mar. 2008 & F. Fang, Thein Win \\
\hline 16 Feb. 2004 & M. Charlton \\
\hline 08 Apr. 2007 & P. Borsa \\
\hline 12 May 2006 & DIFS \\
\hline Jul.-Nov. 2008 & D. Nyingi \\
\hline Jul.-Nov. 2008 & D. Nyingi \\
\hline 09 May 2006 & DIFS \\
\hline 10 May 2006 & DIFS \\
\hline Oct. 2008 & P. Durville, N. Hubert \\
\hline 01 July 2008 & B.W. Jamandre \\
\hline 2007 & H. Ashanti \\
\hline 25 Feb. 2009 & D. Vo \\
\hline 19 Sep. 2008 & P. Borsa \\
\hline 19 Sep. 2008 & P. Borsa \\
\hline 15 Aug. 2004 & P.-F. Lee \\
\hline- & W.-L. Li \\
\hline 14 Apr. 2010 & B. Barbanti \\
\hline 07 May 2010 & B. Barbanti \\
\hline 14 Sep. 2010 & C. Oliveira \\
\hline 14 Sep. 2010 & C. Oliveira \\
\hline 14 Apr. 2010 & B. Barbanti \\
\hline 21 Dec. 2009 & B. Barbanti \\
\hline 14 Sep. 2010 & c. Oliveira \\
\hline 20 June 2010 & B. Barbanti \\
\hline 14 Apr. 2010 & B. Barbanti \\
\hline 14 Apr. 2010 & B. Barbanti \\
\hline 14 Sep. 2010 & C. Oliveira \\
\hline 14 Sep. 2010 & C. Oliveira \\
\hline 16 Feb. 2012 & C. Oliveira \\
\hline 16 Feb. 2012 & C. Oliveira \\
\hline 16 Feb. 2012 & C. Oliveira \\
\hline 16 Feb. 2012 & C. Oliveira \\
\hline 14 Apr. 2010 & B. Barbanti \\
\hline 13 Sep. 2010 & C. Oliveira \\
\hline 11 Dec. 2008 & C. Oliveira \\
\hline 28 Mar. 2006 & R. Causse et al. \\
\hline 15 Mar. 2006 & S. Planes \\
\hline 15 Mar. 2006 & S. Planes \\
\hline 15 Mar. 2006 & S. Planes \\
\hline 15 Mar. 2006 & S. Planes \\
\hline 15 Mar. 2006 & S. Planes \\
\hline & E. Keskin \\
\hline
\end{tabular}

Durand et al. 2012b
Durand et al. 2012b
Durand et al. 2012b
Durand et al. 2012b
Durand et al. 2012b
Durand et al. 2012b
Durand et al. 2012b
Durand et al. 2012b
Durand et al. 2012b
Durand et al. 2012b
Durand et al. 2012b
Durand et al. 2012b
Durand et al. 2012b
Durand et al. 2012b
Durand et al. 2012b
Durand et al. 2012b
Durand et al. 2012b
Durand et al. 2012b
Durand et al. 2012b
Durand et al. 2012b
Durand et al. 2012b
Durand et al. 2012b
Durand et al. 2012b
Durand et al. 2012b
Durand et al. 2012b
Durand et al. 2012b
Durand et al. 2012b
Durand et al. 2012b
Durand et al. 2012b
Durand et al. 2012b
Durand et al. 2012b
Durand et al. 2012b
Durand et al. 2012b
Durand et al. 2012b
Durand et al. 2012b

Phylogeny

Mugil curema

Mugil hospes

Phylogeny Mugilincilis

Phylogeny Mugincils

Phylogeny Mugilliza

Phylogeny Mugilliza

Phylogeny Mugil rubrioculus

Phylogeny

Phylogeny Mugil trichodon

Phylogeny Myxus capensis

Myxus elongatus

Phylogeny Neomyus leuciscus

Neomyxus leuciscus

Phylogeny

Phylogeny

Phylogeny Liza melinoptera

Phylogeny Rhinomugil corsula

Rhinomugil nasutus

Phylogeny Sicamugil cascasia

Phylogeny Sicamugil hamiltonii

Trachystoma petardi

Phylogeny Valamugil buchanani

Phylogeny Valamugl buchanani

Phylogeny Valamugi buchanani

Phylogeny Valamign buchanani

Phylogeny Valamugir robusus

Phylogeny Valamugit robustus

Phylogeny Valamugil robustus

Phylogeny Valamugil sp.

Phylogeny Valamugil sp.

Phylogeny Valamugil sp.

Phylogeny Valamugilsp.

Phylogeny Valamugil sp.

Phylogeny Valamugil sp.

Liza haematocheila

Mugil curema

Mugil curema
Mugil curema

Mugil curema

Mugil curema

Mugil curema

Mugil curema

Mugil curema

Mugil curema

Mugil curema

Mugil curema

Mugil curema

Mugil curema

Mugil hospes

Mugil hospes

Mugil hospes

Mugil liza

Mugil liza

Mugil liza

Crenimugil crenilabis

Ellochelon vaigiensis

Ellochelon vaigiensis

Moolgarda engeli

Moolgarda engeli

Moolgarda engeli

Liza aurata
Mugil sp. 0

ugil sp. 0

Mugil sp. R

ugil incilis

Mugilliza

Mugilliza

Mugil rubrioculus

Mugil sp. P

odon

seudomyxus capensis

yns elus

Neomyxus leuciscus

Dedalechilus labeo

Planiliza sp. B

Rhinomugil corsula

Squalomgin nasulus

Sicamugil hamitonii

Trachystoma petardi

Trachystoma petardi

Crenimugil buchanani

Crenimugil buchanani

Crenimugil buchanan

Osteomugil robustus

Osteomugil robustus

Osteomugil robustus

Osteomugil perusii

Osteomugil perusil

Osteomugil perus

Crenimugil sp. C

Crenimugil sp. D

Crenimugil sp. D

Crenimugil sp. D

Muliza haematocheila

Mugil curema

Mugil curema

ugil curema

Mugil curema

Mugil curema

Mugil curema

Mugil curema

Mugil curema

Mugil curema

Mugil curema

Mugil curema

Mugil curema

Mugil brevirostris

Mugil brevirostris

Mugil brevirostris

Mugil liza

Mugil liza

Mugil liza

Crenimugil crenilabis

Ellochelon vaigiensis

Ellochelon vaigiensis

Osteomugil engell

Osteomugil engell

Osteomugil engel 
Turkey

FCFOPB060-08

FCFOPBOPB $060-06$

FCFOPB083-05

BZLW4430

BZLW4506

BZLW4432

BZLW4431

BZLW4177

BZLW6045

BZLW6036

BZLWD7877

BZLW7069

BZLW7032
CURA8417

CURA8418

CURA8419

SMSA7546

SMSA7547

SMSA7001

SMSA7002

SMSA7003

SMSA7004
SMSA7005

SMSA7006

SMSA7013

SMSA7008

SMSA7007

SMSA7009
SMSA7010

SMSA7011

SMSA7012

TOB9377

TOB9379
TOB9378

PTR91

PTR88

PTR89

PTR90

PTR92

DOF113
LBPV48504

LBPV48501

LBP6848 36406

LBP6848 36409

LBP6848 36407

LBP6848 36410

LBP6848 36408

LBP9923 40546

BP6849 33444

LBP7572 35509

LBP5806 28259

LBP5806 28261

LBP6849 33446

LBP6023 27891

LBP5806 28258

LBP5806 28262

LBP6849 32767
LBP5671 36402
$39.92 \mathrm{~N} 9.00$ W, Portugal

. $.03 \mathrm{~N} 9.98 \mathrm{~W}$, Portugal

$25 \mathrm{~N} 8.98 \mathrm{~W}$, Portuga

41.25 N.00 W, Portuga

$16.802 \mathrm{~N} 88.082 \mathrm{~W}$, Carrie Bow Cay, Belize

16.802 N 88.082 W, Carrie Bow Cay, Belize

16.802 N 88.082 W, Carrie Bow Cay, Belize

16.802 N 88.082 W, Carrie Bow Cay, Belize

1682 N 88082 W Care Bow Cay Belize

(16.8.

17.45 N 88.1167 W, Belize City Fishmarket, Belize

16.802 N 88.082 W, Belize

, Carrie Bow Cay, Belize

Curacao

121375 N 08.9724 W Piscado Bay mangroves, Curacao

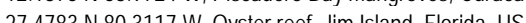

$27.4783 \mathrm{~N} 80.3117$ W. Oyster reef, Jim Island, Florida, USA

$27.6449 \mathrm{~N} 80.3692 \mathrm{~W}$, seine tow, Florida, USA

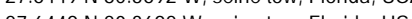

$27.0449 \mathrm{~N} 80.3092 \mathrm{~W}$, seine TOW, FId, USA

$27.6449 \mathrm{~N} 80.3692 \mathrm{~W}$, sene tow, Fonda, USA

27.6449 N 80.3092 W, Se, TW, Fond, USA

$27.6449 \mathrm{~N} 80.3692 \mathrm{~W}$, Se, Tow, Fonda, USA

$27.0449 \mathrm{~N} 80.3092 \mathrm{~W}$, Seine Tow, Fonda, USA

$27.0449 \mathrm{~N} 80.3692 \mathrm{~W}$, seine tow, Fonda, USA

$27.6449 \mathrm{~N} 80.3692 \mathrm{~W}$, seine tow, Florida, USA

$27.6449 \mathrm{~N} 80.3692 \mathrm{~W}$, seine tow, Florida, USA

$27.6449 \mathrm{~N} 80.3692 \mathrm{~W}$, seine tow, Florida, USA

$27.6449 \mathrm{~N} 80.3692 \mathrm{~W}$, seine tow, Florida, USA

11.1466 N 60.783 W, Petit Trou, Tobago

111466 N 60.783 W. Petit Trou, Tobago

Rio Guayalejo, Xicotencatt, Tamaulipas, Mexico

Rio Guayalejo, Xicotencatl, Tamaulipas, Mexico

Rio Gayajo, Xiconcall, Tamaulipas, Mexico

Rio Guayalejo, Xicotencatl, Tamaulipas, Mexico

24.46 N 93.01 E, India

Rio de Janeiro, Brazi

\begin{tabular}{|c|c|}
\hline & E. Keskin \\
\hline & E. Keskin \\
\hline 22 Oct. 2005 & J. Pastor \\
\hline 26 Oct. 2005 & J. Pastor \\
\hline 05 Nov. 2005 & J. Pastor \\
\hline 22 Oct. 2005 & J. Pastor \\
\hline 05 Nov. 2005 & J. Pastor \\
\hline 05 Mar. 2005 & L.A. Weigt \\
\hline 06 Mar. 2005 & L.A. Weigt \\
\hline 05 Mar. 2005 & L.A. Weigt \\
\hline 05 Mar. 2005 & L.A. Weigt \\
\hline 28 Feb. 2004 & L.A. Weigt \\
\hline 31 Mar. 2006 & C.C. Baldwin \\
\hline 30 Mar. 2006 & C.C. Baldwin \\
\hline 04 Oct. 2007 & local fishermen \\
\hline 13 Jan. 2007 & L.A. Weigt \\
\hline 12 Jan. 2007 & L.A. Weigt \\
\hline 15 Mar. 2004 & C.C. Baldwin \\
\hline 15 Mar. 2004 & C.C. Baldwin \\
\hline 15 Mar. 2004 & C.C. Baldwin \\
\hline - & C.C. Baldwin \\
\hline - & C.C. Baldwin \\
\hline - & C.C. Baldwin \\
\hline - & C.C. Baldwin \\
\hline - & C.C. Baldwin \\
\hline & C.C. Baldwin \\
\hline - & C.C. Baldwin \\
\hline - & C.C. Baldwin \\
\hline - & C.C. Baldwin \\
\hline - & C.C. Baldwin \\
\hline - & C.C. Baldwin \\
\hline - & C.C. Baldwin \\
\hline - & C.C. Baldwin \\
\hline - & C.C. Baldwin \\
\hline - & C.C. Baldwin \\
\hline 20 Mar. 2009 & C.C. Baldwin \\
\hline 20 Mar. 2009 & C.C. Baldwin \\
\hline 20 Mar. 2009 & C.C. Baldwin \\
\hline & E. Soto-Galera \\
\hline - & E. Soto-Galera \\
\hline - & E. Soto-Galera \\
\hline - & E. Soto-Galera \\
\hline- & E. Soto-Galera \\
\hline 23 Mar. 2011 & S.K. Ghosh \\
\hline 19 Oct. 2010 & C. Oliveira \\
\hline 19 Oct. 2010 & C. Oliveira \\
\hline - & - \\
\hline - & . \\
\hline - & - \\
\hline - & - \\
\hline - & - \\
\hline - & \\
\hline - & - \\
\hline - & - \\
\hline & \\
\hline - & 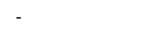 \\
\hline - & \\
\hline & \\
\hline - & \\
\hline & \\
\hline & - \\
\hline
\end{tabular}

Barcoding

Barcoding

Barcoding

Barcoding

Barcoding

Barcoding

Barcoding

Barcoding

Barcoding

Barcoding

Barcodin

Barcoding

Barcoding

Barcoding

Barcoding

Barcoding

Barcoding

Barcoding

Barcoding

Barcoding

Barcoding

Barcoding

Barcodin

Barcoding

Barcoding

Barcoding

Barcoding

Barcoding

Barcoding

Barcoding

Barcoding

Barcoding

Barcoding

Barcoding

Barcoding

Barcoding

Barcoding

Barcoding

Barcoding

unpubl.

unpubl.

unpubl.

unpubl.

unpubl.

unpubl.

Siccha-Ramirez at. 2014 Phylogeography Mugil liza

Siccha-Ramirez et al. 2014 Phylogeography Mugil liza

Siccha-Ramirez et al. 2014 Phylogeography Mugil liza

Siccha-Ramirez et al. 2014 Phylogeography Mugil liza

Siccha-Ramirez et al. 2014 Phylogeography Mugil liza

Siccha-Ramirez et al. 2014 Phylogeography Mugil liza

Siccha-Ramirez et al. 2014 Phylogeography Mugil liza

Siccha-Ramirez et al. 2014 Phylogeography Mugil liza

Siccha-Ramirez et al. 2014 Phylogeography Mugil liza iza carinata

Mugil cephalus

Lza ramado

rizamado

gil sp.

ugil sp.

Mugilidae sp.

Mugilidae sp.

ugilidae sp.

guldae sp.

Mugil curema
Mugil curema

Mugil sp.

ugil cephalus

uril cephalus

Mugil cephalus

ugil cephalus

ugil curema

ugil curema

Mugil curema

Mugil incilis

Mugil incilis

gonostomus montic

-

gonostomus monticola

Mugil liza

Mugil cephalus

Mugil cephalus

Mugil cephalus

ugil cephalus

gil cephalus
Planiliza macrolepis

Mugil sp. I

Chelon ramado

Chelon ramado

Chelon ramado

Chelon ramado

Mugil rubrioculus

Mugil rubrioculus

Mugil curema

Mugil curema

Mugil curema

Mugil sp R

Mugil sp. R

Mugil curema

Mugil curema

Mugil rubrioculus

Mugil rubrioculus

Mugil sp. B

Mugil sp. B

Mugil sp. B

Mugil sp. B

Mugil sp. B

Mugil sp. B

Mugil curema

Mugil curema

mugil curema

Mugil curema

Mugi incilis

Mugil incilis

Mugil incilis

Mugil incilis

Dajaus sp.

Dajaus sp.

Dajaus sp.

Rhinomugil corsul

Mugil liza

Mugil liza

Chelon labrosus

Chelon labrosus

Chelon labrosus

Chelon labrosus

Chelon labrosus

Mugil liza

Mugil liza

Mugil liza

Mugil liza

Mugil liza

Mugil liza

Mugil liza

Mugil liza

Mugil liza

Mugil liza

Mugil liza 


\begin{tabular}{|c|c|c|c|}
\hline JX185151 & - & LBP5671 36404 & Uruguay \\
\hline JX185152 & - & LBP7572 35507 & Brazil \\
\hline JX185153 & . & LBP7572 35511 & Brazil \\
\hline JX185154 & . & LBP5671 36405 & Uruguay \\
\hline JX185155 & - & LBP5671 36401 & Uruguay \\
\hline JX185156 & . & LBP5671 36403 & Uruguay \\
\hline JX185157 & - & LBP6023 27887 & Brazil \\
\hline JX185158 & . & LBP6849 33447 & Brazil \\
\hline JX185159 & . & LBP7572 35510 & Brazil \\
\hline JX185160 & . & LBP6279 27726 & Argentina \\
\hline JX185161 & . & LBP6023 27892 & Brazil \\
\hline JX185162 & . & LBP6023 27886 & Brazil \\
\hline JX185163 & - & LBP6279 27720 & Argentina \\
\hline JX185164 & - & LBP6064 30219 & Venezuela \\
\hline JX185165 & - & LBP6064 30227 & Venezuela \\
\hline JX185166 & . & LBP6064 30089 & Venezuela \\
\hline JX185167 & . & LBP9108 42759 & Brazil \\
\hline JX185168 & . & LBP9108 42761 & Brazil \\
\hline JX185169 & - & LBP9108 42760 & Brazil \\
\hline JX185170 & - & LBP6849 33445 & Brazil \\
\hline JX185171 & . & LBP5806 28260 & Brazil \\
\hline JX185172 & . & LBP6064 30221 & Venezuela \\
\hline JX185173 & . & LBP6279 27706 & Argentina \\
\hline JX185174 & . & LBP6279 27709 & Argentina \\
\hline JX185175 & - & LBP6279 27717 & Argentina \\
\hline JX185176 & - & LBP7572 35508 & Brazil \\
\hline JX185177 & - & LBP6023 27893 & Brazil \\
\hline JX185178 & . & LBP6066 30105 & Venezuela \\
\hline JX185179 & . & LBP6066 30770 & Venezuela \\
\hline JX185180 & . & LBP9059 42702 & Brazil \\
\hline JX185181 & - & LBP 786 & Venezuela \\
\hline JX185182 & - & LBP9061 42657 & Brazil \\
\hline JX185183 & - & LBP 781 & Venezuela \\
\hline JX185184 & - & LBP 782 & Venezuela \\
\hline JX185185 & . & LBP 785 & Venezuela \\
\hline JX185186 & - & LBP 780 & Venezuela \\
\hline JX185187 & - & LBP 788 & Venezuela \\
\hline JX185188 & - & LBP9059 42663 & Brazil \\
\hline JX185189 & - & LBP9059 42703 & Brazil \\
\hline JX185190 & - & LBP9059 42662 & Brazil \\
\hline JX185191 & . & LBP9061 42658 & Brazil \\
\hline JX185192 & - & LBP9059 42701 & Brazil \\
\hline JX185193 & . & LBP6065 30149 & Venezuela \\
\hline JX185194 & - & LBP6129 29541 & Venezuela \\
\hline JX185195 & - & LBP6129 29542 & Venezuela \\
\hline JX185196 & - & LBP6065 30070 & Venezuela \\
\hline JX185197 & . & LBP6065 30153 & Venezuela \\
\hline JX185198 & . & LBP10004 46937 & Brazil \\
\hline JX185199 & - & LBP10051 40560 & Brazil \\
\hline JX185200 & . & LBP10051 40558 & Brazil \\
\hline JX185201 & - & LBP10004 46938 & Brazil \\
\hline JX185202 & - & LBP10004 40566 & Brazil \\
\hline JX185203 & . & LBP10051 40506 & Brazil \\
\hline JX185204 & . & LBP10051 40557 & Brazil \\
\hline JX185205 & . & LBP10004 46939 & Brazil \\
\hline JX185206 & - & LBP10051 40559 & Brazil \\
\hline JX185207 & - & LBP6128 29540 & Venezuela \\
\hline JX185208 & - & LBP9060 42656 & Brazil \\
\hline JX185209 & - & LBP9060 42653 & Brazil \\
\hline JX185210 & . & LBP6063 30086 & Venezuela \\
\hline JX185211 & . & LBP6063 30088 & Venezuela \\
\hline JX185212 & - & LBP9060 42655 & Brazil \\
\hline
\end{tabular}

Siccha-Ramirez et al. 2014 Phylogeography Mugil liza Siccha-Ramirez et al. 2014 Phylogeography Mugil liza Siccha-Ramirez et al. 2014 Phylogeography Mugil liza Siccha-Ramirez et al. 2014 Phylogeography Mugil liza Siccha-Ramirez et al. 2014 Phylogeography Mugil liza Siccha-Ramirez et al. 2014 Phylogeography Mugil liza Siccha-Ramirez et al. 2014 Phylogeography Mugil liza Siccha-Ramirez et al. 2014 Phylogeography Mugil liza Siccha-Ramirez et al 2014 Phylogeoraphy Mugl liza Siccha-Ramirez et al 2014 Phylogeoraphy Muglliza Siccha-Ramirez et al 2014 Phylogeoraphy Muglliza Siccha-Ramizz 2014 Phylogeography Muglliza Siccha-Ramirez et al. 2014 Phylogeography Mugil liza Siccha-Ramirez et al. 2014 Phylogeography Mugil liza Siccha-Ramirez et al. 2014 Phylogeography Mugil liza Siccha-Ramirz el 2014 Phyloneoghy Mulliza Siccha-Ramiez etal 2014 Phylogeography Mugiliza Siccha-Ramiez et 2014 Phylogegraphy Muiliza Siccha-Ramiz et 2014 Phylogegaphy Mul SicchaSiccha-Pamirez t 2014 Phyogrog Muliza Siccha-Ramirez al 2014 Phyog Siccha-Ramirez et al 2014 Phylogography Mugiza Siccha-Ramirez al 2014 Phylog Siccha-Ramiez el. 2014 Phylogophy Mul iza Siccha-Ramirez et al. 2014 Phylogeography Mugil liza Siccha-Ramirez et al. 2014 Phylogeography Mugil liza Siccha-Ramirez et al. 2014 Phylogeography Mugil trichodon Siccha-Ramiez el al. 2014 Phylogeography Mugil trichodon Siccha-Ramirez et al. 2014 Phylogeography Mugil incils Siccha-Ranirez et al. 2014 Phylogeography Mugin incilis Siccha-Ramicz et al. 2014 Phlogeography Mugil incilis Siccha-Ramirez et al. 2014 - Phylogeography Mugh incilis Siccha-Ramirez et al. 2014 Phylogeography Mugil incilis Siccha-Ramirez et al. 2014 Phylogeography Mugil incilis Siccha-Ramirez et al. 2014 Phylogeography Mugil incilis Siccha-Ramilez el al. 2014 Phylogeography Mugil incilis Siccha-Ramirez et al. 2014 Phylogeography Mugil incilis Siccha-Ramirez et al. 2014 Phylogeography Mugil incilis Siccha-Ramirez et al. 2014 Phylogeography Mugil incilis $\begin{array}{ll}\text { Siccha-Ramirez et al. } 2014 & \text { Phylogeography Mugil inclils } \\ \text { Siccha-Ramirez et al } 2014 \text { Phylogeography Mugil incilis }\end{array}$ Siccha-Ramirez et al. 2014 Phylogeography Mugil curema Siccha-Ramirez et al. 2014 Phylogeography Mugil curema Siccha-Ramirez et al. 2014 Phylogeography Mugil curema Siccha-Ramirez et al. 2014 Phylogeography Mugil curema Siccha-Ramirez et al. 2014 Phylogeography Mugil curema Siccha-Ramirez et al. 2014 Phylogeography Mugil curema Siccha-Ramilez et al. 2014 Phylogeography Mugil curema Siccha-Ramirez et al. 2014 Phylogeography Mugil curema Siccha-Ramirez et al. 2014 Phylogeography Mugil curema Siccha-Ramirez et al. 2014 Phylogeography Mugil curema Siccha-Ramirez et al. 2014 Phylogeography Mugil curema Siccha-Ramirez et al. 2014 Phlogeography Mugil curema Siccha-Ramirez et al. 2014 Phylogeography Mugil curema Siccha-Ramirez el al. 2014 Phylogeography Mugil curema Siccha-Ramirez et al. 2014 Phylogeography Mugil sp. Siccha-Ramirez et al. 2014 Phylogeography Mugi rubrioculus Siccha-Ramirez et al. 2014 Phylogeography Mugil rubrioculus Siccha-Ramirez et al. 2014 Phylogeography Mugil rubrioculus Siccha-Ramirez et al. 2014 Siccha-Ramirez et al. 2014 Phylogeography Mugil rubrioculus
Mugil liza

Mugil liza

Mugil liza

Mugilliza

Mugilliza

Musiriza

Mugil liza

Musiliza

Musiliza

Muri liza

Musiriza

Mugiliza

Muiliza

Mugiliza

Mugiza

Mugil liza

Mugiza

Muri liza

Mugil liza

Mugil liza

Mugil Iza

Mugil liza

Mugin liza

Mugiliza

Mugil liza

Mugit trichodon

Mugil trichodon

Mugil incilis

Mugil incilis

Mugil incilis

Mugil incilis

Mugil incilis

Mugil incilis

Mugil incilis

Mugil inclils

Mugil incilis

Mugil incilis

Mugil incilis

Mugil incilis

Mugil incilis

Mugil incilis

Mugil margaritae

Mugil margaritae

Mugil margaritae

Mugil margaritae

Mugil margarita

Mugil curema

Mugil curema

Mugil curema

Mugil curema

Mugil curema

Mugil curema

Mugil curema

Mugil curema

Mugil curema

Mugil sp. R

Mugil rubrioculus

Mugil rubrioculus

Mugil rubrioculus

Mugil rubrioculus
Mugil rubrioculus 


\begin{tabular}{|c|c|c|c|}
\hline JX185213 & - & LBP6063 30087 & Venezuela \\
\hline JX185214 & - & LBP9060 42652 & Brazil \\
\hline JX185215 & - & LBP9060 42654 & Brazil \\
\hline JX185216 & - & LBP6061 42659 & Brazil \\
\hline JX185217 & - & LBP6061 42660 & Brazil \\
\hline JX185218 & - & LBP6061 42661 & Brazil \\
\hline JX185219 & - & LBP6061 30201 & - \\
\hline JX260846 & - & LZYN01 & Yanum estuary, Andhra Pradesh, India \\
\hline JX260972 & - & SCYN09 & Yanum estuary, Andhra Pradesh, India \\
\hline JX559532 & Mgal6446 & - & Bahia Divine, Isla Santa Cruz, Galapagos Is., Ecuador \\
\hline JX559533 & Mgal6450 & - & Bahia Divine, Isla Santa Cruz, Galapagos Is., Ecuador \\
\hline JX559534 & Xt6435 & . & Bahia Divine, Isla Santa Cruz, Galapagos Is., Ecuador \\
\hline JX559535 & $x+6445$ & - & Bahia Divine, Isla Santa Cruz, Galapagos Is., Ecuador \\
\hline JX983355 & - & NF760 & Bharuch, Gujarat, India \\
\hline JX983356 & - & NF762 & Bharuch, Gujarat, India \\
\hline JX983357 & - & NF761 & Bharuch, Gujarat, India \\
\hline JX983367 & - & NF569 & Bharuch, Gujarat, India \\
\hline JX983368 & . & NF549 & Bharuch, Gujarat, India \\
\hline JX983369 & - & NF574 & Bharuch, Gujarat, India \\
\hline JX983482 & - & NF759 & Bharuch, Gujarat, India \\
\hline JX983483 & - & NF568 & Bharuch, Gujarat, India \\
\hline JX983495 & - & NF550 & Bharuch, Gujarat, India \\
\hline JX983496 & - & NF565 & Bharuch, Gujarat, India \\
\hline КС349864 & PB973 & - & supermarket, Castenaso, Italy \\
\hline KC500813 & - & TR840EK & Izmir Bay, Turkey \\
\hline KC500814 & - & TR851EK & Izmir Bay, Turkey \\
\hline KC500815 & - & TR841EK & Izmir Bay, Turkey \\
\hline KC500816 & - & TR859EK & Izmir Bay, Turkey \\
\hline KC500817 & - & TR858EK & Izmir Bay, Turkey \\
\hline KC500818 & - & TR856EK & Izmir Bay, Turkey \\
\hline KC500819 & . & TR855EK & Izmir Bay, Turkey \\
\hline KC500820 & - & TR854EK & Izmir Bay, Turkey \\
\hline KC500821 & - & TR853EK & Izmir Bay, Turkey \\
\hline KC500822 & - & TR852EK & Izmir Bay, Turkey \\
\hline KC500823 & - & TR857EK & Izmir Bay, Turkey \\
\hline KC500824 & - & TR842EK & Izmir Bay, Turkey \\
\hline KC500825 & . & TR843EK & Izmir Bay, Turkey \\
\hline KC500826 & - & TR850EK & Izmir Bay, Turkey \\
\hline KC500827 & - & TR844EK & Izmir Bay, Turkey \\
\hline KC500828 & - & TR845EK & Izmir Bay, Turkey \\
\hline KC500829 & - & TR846EK & Izmir Bay, Turkey \\
\hline KC500830 & - & TR847EK & Izmir Bay, Turkey \\
\hline KC500831 & - & TR848EK & Izmir Bay, Turkey \\
\hline KC500832 & . & TR849EK & Izmir Bay, Turkey \\
\hline KC500833 & - & TR868EK & Iskenderun Bay, Turkey \\
\hline KC500834 & - & TR870EK & Iskenderun Bay, Turkey \\
\hline KC500835 & - & TR879EK & Iskenderun Bay, Turkey \\
\hline KC500836 & - & TR878EK & Iskenderun Bay, Turkey \\
\hline KC500837 & - & TR877EK & Iskenderun Bay, Turkey \\
\hline KC500838 & - & TR876EK & Iskenderun Bay, Turkey \\
\hline KC500839 & . & TR875EK & Iskenderun Bay, Turkey \\
\hline KC500840 & - & TR874EK & Iskenderun Bay, Turkey \\
\hline KC500841 & - & TR873EK & Iskenderun Bay, Turkey \\
\hline KC500842 & - & TR872EK & Iskenderun Bay, Turkey \\
\hline KC500843 & - & TR871EK & Iskenderun Bay, Turkey \\
\hline KC500844 & - & TR861EK & Iskenderun Bay, Turkey \\
\hline KC500845 & - & TR860EK & Iskenderun Bay, Turkey \\
\hline KC500846 & - & TR862EK & Iskenderun Bay, Turkey \\
\hline KC500847 & - & TR863EK & Iskenderun Bay, Turkey \\
\hline KC500848 & - & TR864EK & Iskenderun Bay, Turkey \\
\hline KC500849 & - & TR865EK & Iskenderun Bay, Turkey \\
\hline KC500850 & - & TR866EK & Iskenderun Bay, Turkey \\
\hline
\end{tabular}

\begin{tabular}{|c|c|}
\hline & - \\
\hline - & - \\
\hline - & - \\
\hline - & - \\
\hline - & - \\
\hline - & $\cdot$ \\
\hline- & V. Kalyankar \\
\hline 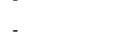 & $\begin{array}{l}\text { V. Kalyankar } \\
\text { V. Kar }\end{array}$ \\
\hline 15 June 2011 & T. Ballesteros, N. Tirado \\
\hline 15 June 2011 & T. Ballesteros, N. Tirado \\
\hline 15 June 2011 & T. Ballesteros, N. Tirado \\
\hline 15 June 2011 & T. Ballesteros, N. Tirado \\
\hline - & R.A. Jamdade \\
\hline - & R.A. Jamdade \\
\hline & R.A. Jamdade \\
\hline 20 Oct. 2010 & R.A. Jamdade \\
\hline 20 Oct. 2010 & R.A. Jamdade \\
\hline 20 Oct. 2010 & R.A. Jamdade \\
\hline - & R.A. Jamdade \\
\hline 20 Oct. 2010 & R.A. Jamdade \\
\hline 20 Oct. 2010 & R.A. Jamdade \\
\hline 20 Oct. 2010 & R.A. Jamdade \\
\hline - & P. Bartosova \\
\hline - & E. Keskin \\
\hline - & E. Keskin \\
\hline - & E. Keskin \\
\hline - & E. Keskin \\
\hline - & E. Keskin \\
\hline - & E. Keskin \\
\hline - & E. Keskin \\
\hline - & E. Keskin \\
\hline . & E. Keskin \\
\hline - & E. Keskin \\
\hline - & E. Keskin \\
\hline - & E. Keskin \\
\hline - & E. Keskin \\
\hline - & E. Keskin \\
\hline - & E. Keskin \\
\hline . & E. Keskin \\
\hline - & E. Keskin \\
\hline - & E. Keskin \\
\hline - & E. Keskin \\
\hline - & E. Keskin \\
\hline - & E. Keskin \\
\hline - & E. Keskin \\
\hline - & E. Keskin \\
\hline - & E. Keskin \\
\hline - & E. Keskin \\
\hline - & E. Keskin \\
\hline - & E. Keskin \\
\hline - & E. Keskin \\
\hline - & E. Keskin \\
\hline - & E. Keskin \\
\hline - & E. Keskin \\
\hline - & E. Keskin \\
\hline & E. Keskin \\
\hline - & E. Keskin \\
\hline - & E. Keskin \\
\hline - & E. Keskin \\
\hline & E. Keskin \\
\hline- & E. Keskin \\
\hline
\end{tabular}

Siccha-Ramirez et al. 2014 Phylogeography Mugil rubrioculus Siccha-Ramirez et al. 2014 Phylogeography Mugil rubrioculus Siccha-Ramirez et al. 2014 Phylogeography Mugil rubrioculus Siccha-Ramirez et al. 2014 Phylogeography Mugil hospes Siccha-Ramirez et al. 2014 Phylogeography Mugil hospes Siccha-Ramirez et al. 2014 Phylogeography Mugil hospes Siccha-Ramirez et al. 2014 Phylogeography Mugil curema

unpubl.

Durand et al. 2012a

Durand et al. 2012a

Durand et al. 2012

Durand et al. 2012

Khedkar et al. 2014

Khedkar et al. 2014

Khedkar et al. 2014

Khedkar et al. 2014

Kheckar et al. 2014

Khedkar et al. 2014

Khedkar et al. 2014

Khedkar et al. 2014

Khedkar et al. 2014

Khedkar et al. 2014

Keskin and Atar 2013

Keskin and Atar 2013

Keskin and Atar 2013

Keskin and Atar 2013

Keskin and Atar 2013

Keskin 2013

Keskin and Atar 2013

Keskin and Atar 2013

Keskin and Atar 2013

Keskin and Atar 2013

Keskin and Atar 2013

Keskin and Atar 2013

Keskin and Atar 2013

Keskin and Atar 2013

Keskin and Atar 2013

Keskin and Atar 2013

Keskin and Atar 2013

Keskin and Atar 2013

Keskin and Atar 2013

Keskin and Atar 2013

Keskin and Atar 2013

Keskin and Atar 2013

Keskin and Atar 2013

Keskin and Atar 2013

Keskin and Atar 2013

Keskin and Atar 2013

Keskin and Atar 2013

Keskin and Atar 2013

Keskin and Atar 2013

Keskin and Atar 2013

Keskin and Atar 2013

Keskin and Atar 2013

Keskin and Atar 2013

Keskin and Atar 2013

Keskin and Atar 2013

Keskin and Atar 2013

Keskin and Atar 2013
Keskin and Atar 2013
Barcoding Chelon parsia

Barcoding Sicamugil cascasia

Phylogeny Mugil cephalus

Phylogeny Mugic cephalus

Phylogeny Xenomugit thoburn

Barcoding Liza klunzingeri

Barcoding Liza klunzingeri

Barcoding Liza klunzingeri

Barcoding Mugil cephalus

Barcoding Mugil cephalus

Mugil cephalus

Barcoding Rhomugil corsula

Sicamugi cascasia

Sicamugil cascasia

Bacoding

Barcoding

Barcoding

Barcoding

Barcoding

Barcoding

Barcoding

Barcoding

Barcoding

Barcoding

Barcoding

Barcoding

Barcoding

Barcoding

Barcoding

Barcoding

Barcoding

Barcoding

Barcoding

Barcoding

Barcoding

Barcoding

Barcoding

Barcoding

Barcodin

Barcoding

Barcoding

Barcoding

Barcoding

Barcoding

Barcoding

Barcodin

Barcoding
Barcoding
Sicamugil casca
Liza ramado

Liza ramado

Liza aurata

Liza aurata

Liza aurata

Liza aurata

Liza aurata

Liza aurata

Liza aurata

Liza aurata

Liza aurata

Liza aurata

Liza aurata

Liza aurata

Liza aurata

Liza aurata

Liza aurata

Liza aurata

Liza aurata

Liza aurata

Liza carinata

Liza carinata

Liza carinata

Liza carinata

Liza carinata

Liza carinata

Liza carinata

Liza carinata

Liza cammata

Liza carinata

Liza carinata

Liza carina

izzarinata

Liza cannata

Liza carinata

Liza carinata
Mugil rubrioculus ugil rubrioculus ugil rubrioculus

Mugil brevirostris

Mugil brevirostris

Mugil brevirostris

Mugil curema

Osteomugil sp.

Mugil sp. Q

Mugil sp. Q

gil thoburni

Mugil thoburni

Planiliza sp. A

Planiliza sp. A

Planiliza sp. A

Planiliza SP. D

Planiliza sp. D

Rhinomugil corsu

Planiliza sp.

Planiliza sp.

Chelon ramado

Chelon auratus

Chelon auratus

Chelon auralus

Chelon auratus

Chelon auratus

Chelon auratus

Chelon auratus

Chelon auratus

Chelon auratus

Chelon auratus

Chelon auratus

Chelon auratus

Chelon auratus

Chelon auratus

Chelon auratus

Chelon auratus

Chelon auratus

Chelon auratus

Chelon auratus

Chelon auratus

Planiliza macrolepis

Planiliza macrolepis

Planiliza macrolepis

Planiliza macrolepis

Planiliza macrolepis

Planiliza macrolepis

Planiliza macrolepis

Planilza macrolepis

Planilza macrolepis

Planiliza macrolepis

Planiliza macrolepis

Planiliza macrolepis

Planiliza macrolepis

Planiliza macrolepis

Planiliza macrolepis 


\begin{tabular}{|c|c|c|c|c|c|}
\hline KC500851 & - & TR867EK & Iskenderun Bay, Turkey & - & E. Keskin \\
\hline KC500852 & - & TR869EK & Iskenderun Bay, Turkey & - & E. Keskin \\
\hline KC500933 & - & TR976EK & Yumurtallik, Turkey & - & E. Keskin \\
\hline KC500934 & - & TR975EK & Yumurtallk, Turkey & - & E. Keskin \\
\hline KC500935 & - & TR974EK & Yumurtallk, Turkey & - & E. Keskin \\
\hline KC500936 & - & TR973EK & Yumurtallk, Turkey & - & E. Keskin \\
\hline KC500937 & - & TR972EK & Yumurtallk, Turkey & - & E. Keskin \\
\hline KC500938 & - & TR979EK & Yumurtallk, Turkey & - & E. Keskin \\
\hline KC500939 & - & TR978EK & Yumurtallk, Turkey & - & E. Keskin \\
\hline KC500940 & - & TR977EK & Yumurtalik, Turkey & - & E. Keskin \\
\hline KC500941 & - & TR960EK & Yumurtallk, Turkey & - & E. Keskin \\
\hline KC500942 & - & TR961EK & Yumurtallk, Turkey & - & E. Keskin \\
\hline KC500943 & - & TR962EK & Yumurtallk, Turkey & - & E. Keskin \\
\hline KC500944 & & TR971EK & Yumurtallk, Turkey & - & E. Keskin \\
\hline KC500945 & - & TR970EK & Yumurtallk, Turkey & - & E. Keskin \\
\hline KC500946 & - & TR969EK & Yumurtallk, Turkey & - & E. Keskin \\
\hline KC500947 & - & TR968EK & Yumurtallk, Turkey & - & E. Keskin \\
\hline KC500948 & - & TR967EK & Yumurtallk, Turkey & - & E. Keskin \\
\hline KC500949 & - & TR966EK & Yumurtallk, Turkey & - & E. Keskin \\
\hline KC500950 & - & TR965EK & Yumurtallk, Turkey & - & E. Keskin \\
\hline KC500951 & - & TR964EK & Yumurtallk, Turkey & - & E. Keskin \\
\hline KC500952 & - & TR963EK & Yumurtallik, Turkey & - & E. Keskin \\
\hline KC970393 & - & BOLD:ACD9319 & Pangasinan, Philippines & 21 Dec. 2012 & - \\
\hline KF009605 & - & ARO 164 & Aurora, Philippines & 14 Dec. 2012 & M.J. Rafael, S. Alcantara \\
\hline KF010474 & VcunVel-04 & - & Vellar Estuary, Parangipettai, Tamil Nadu, India & 7 May 2012 & M. Ashiq Ur Rahman \\
\hline KF010475 & MugCoch-08 & - & Cochin backwaters, Kochi, Kerala, India & 12 Jan. 2013 & M. Ashiq Ur Rahman \\
\hline KF010476 & MugCoch-09 & - & Cochin backwaters, Kochi, Kerala, India & 12 Jan. 2013 & M. Ashiq Ur Rahman \\
\hline KF010477 & MugCoch-10 & - & Cochin backwaters, Kochi, Kerala, India & 12 Jan. 2013 & M. Ashiq Ur Rahman \\
\hline KF010478 & MugCoch-12 & - & Cochin backwaters, Kochi, Kerala, India & 12 Jan. 2013 & M. Ashiq Ur Rahman \\
\hline KF010479 & MugCoch-13 & - & Cochin backwaters, Kochi, Kerala, India & 12 Jan. 2013 & M. Ashiq Ur Rahman \\
\hline KF010480 & MugCoch-14 & - & Cochin backwaters, Kochi, Kerala, India & 12 Jan. 2013 & M. Ashiq Ur Rahman \\
\hline KF010481 & MugCoch-15 & - & Cochin backwaters, Kochi, Kerala, India & 12 Jan. 2013 & M. Ashiq Ur Rahman \\
\hline KF010482 & MugCoch-16 & - & Cochin backwaters, Kochi, Kerala, India & 12 Jan. 2013 & M. Ashiq Ur Rahman \\
\hline KF214305 & - & NFGJ573 & Narmada Estuary, Bharuch, Gujarat, India & 9 Dec. 2012 & \\
\hline KF489538 & - & ADC12_222.6 6 & Little Toti estuary, South Africa & 13 Jan. 2010 & S. Weerts \\
\hline KF667528 & - & - & - & - & - \\
\hline KF714973 & - & PGN159 & Pangasinan, Philippines & 13 Jun. 2013 & S. Alcantara, H.J. Juguilon \\
\hline KF714976 & - & PGN213 & Pangasinan, Philippines & 13 Jun. 2013 & S. Alcantara, H.J. Juguilon \\
\hline KF929795 & - & KUT 6844 & South Beach, Port Launay, Mahe, Seychelles & 19 Apr. 2005 & K.A. Moots et al. \\
\hline KF930055 & - & KUT 6842 & South Beach, Port Launay, Mahe, Seychelles & 19 Apr. 2005 & K.A. Moots et al. \\
\hline KF930056 & . & KUT 6496 & Kelly's Beach rock pools, Port Alfred, Eastern Cape, South Africa & 25 Sept. 2004 & K.A. Moots et al. \\
\hline KF930146 & - & KUT 1636 & Ponds on 2 miles on east side of San Luis Pass, Galveston, Texas, $L$ & A 24 Sept. 1995 & L. Balls et al. \\
\hline KF930147 & - & KUT 826 & Dauphin Island, Mobile, Alabama, USA & 14 Jul. 1993 & B. Kuhajda \\
\hline KF930148 & - & KUT 5833 & Deep hole, West side of Turneffe Caye, Belize & 08 Jul. 1999 & T.M. Orrell \\
\hline KF930166 & - & KUT 6497 & Kelly's Beach rock pools, Port Alfred, Eastern Cape, South Africa & 25 Sept. 2004 & K.A. Moots et al. \\
\hline KF930530 & - & KUT 6847 & South Beach, Port Launay, Mahe, Seychelles & 19 Apr. 2005 & K.A. Moots et al. \\
\hline KJ013050 & - & DB 5.2 & Bulacan, Manila Bay, Central Luzon, Luzon, Philippines & 20 Oct. 2012 & M.A.R. Marucot \\
\hline KJ128451 & - & NRM:52307 & 58.0569 N $16.8183 \mathrm{E}$, Sweden & 27 Jul. 2005 & M. Nilsson, S. Nilsson \\
\hline KJ128452 & - & NRM:50609 & Hakefiorden, Sweden & 10 Jun. 2002 & B. Bland \\
\hline KJ202168 & - & BTN9 & Calabarzon, Batangas, Philippines & 1 Jun. 2012 & J.S. Bejarin et al. \\
\hline KJ202169 & - & BTN10 & Calabarzon, Batangas, Philippines & 1 Jun. 2012 & J.S. Bejarin et al. \\
\hline KJ202170 & - & BTN15 & Calabarzon, Batangas, Philippines & 1 Jun. 2012 & J.S. Bejarin et al. \\
\hline KJ202179 & . & BTN17 & Calabarzon, Batangas, Philippines & 1 Jun. 2012 & J.S. Bejarin et al. \\
\hline $\mathrm{KJ} 202180$ & - & BTN16 & Calabarzon, Batangas, Philippines & 1 Jun. 2012 & J.S. Bejarin et al. \\
\hline KJ552298 & Ex06B6 & ZFMK:50573 & 38.395 N 21.435 E, Mediterranean drainage, Greece & $-\quad-2-5$ & - \\
\hline KJ552351 & Ex51D1 & IUSHM 2012-1060 & 36.047 N 35.981 E, Orontes drainage, Turkey & - & - \\
\hline KJ552354 & Ex51D2 & IUSHM 2012-1060 & 36.047 N 35.981 E, Orontes drainage, Turkey & - & - \\
\hline KJ552761 & Ex19E6 & ZFMK:FSJF:ex1540 & 37.528 N 31.269 E, Koeprue drainage, Turkey & - & - \\
\hline KJ552773 & Ex06A5 & ZFMK:50560 & $38.395 \mathrm{~N} 21.435 \mathrm{E}$, Mediterranean drainage, Greece & - & - \\
\hline KJ552776 & Ex22D11 & ZFMK:FSJF:1935 & 33.889 N 6.999 W, Iqem drainage, Morocco & - & . \\
\hline KJ552826 & Ex06B2 & ZFMK:50569 & 38.395 N 21.435 E, Mediterranean drainage, Greece & - & - \\
\hline KJ552858 & Ex06B10 & ZFMK:50577 & 38.395 N 21.435 E, Mediterranean drainage, Greece & - & - \\
\hline
\end{tabular}

\begin{tabular}{|c|c|c|}
\hline Keskin and Atar 2013 & Barcoding & Liza carinata \\
\hline Keskin and Atar 2013 & Barcoding & Liza carinata \\
\hline Keskin and Atar 2013 & Barcoding & Mugil cephalus \\
\hline Keskin and Atar 2013 & Barcoding & Mugil cephalus \\
\hline Keskin and Atar 2013 & Barcoding & Mugil cephalus \\
\hline Keskin and Atar 2013 & Barcoding & Mugil cephalus \\
\hline Keskin and Atar 2013 & Barcoding & Mugil cephalus \\
\hline Keskin and Atar 2013 & Barcoding & Mugil cephalus \\
\hline Keskin and Atar 2013 & Barcoding & Mugil cephalus \\
\hline Keskin and Atar 2013 & Barcoding & Mugil cephalus \\
\hline Keskin and Atar 2013 & Barcoding & Mugil cephalus \\
\hline Keskin and Atar 2013 & Barcoding & Mugil cephalus \\
\hline Keskin and Atar 2013 & Barcoding & Mugil cephalus \\
\hline Keskin and Atar 2013 & Barcoding & Mugil cephalus \\
\hline Keskin and Atar 2013 & Barcoding & Mugil cephalus \\
\hline Keskin and Atar 2013 & Barcoding & Mugil cephalus \\
\hline Keskin and Atar 2013 & Barcoding & Mugil cephalus \\
\hline Keskin and Atar 2013 & Barcoding & Mugil cephalus \\
\hline Keskin and Atar 2013 & Barcoding & Mugil cephalus \\
\hline Keskin and Atar 2013 & Barcoding & Mugil cephalus \\
\hline Keskin and Atar 2013 & Barcoding & Mugil cephalus \\
\hline Keskin and Atar 2013 & Barcoding & Mugil cephalus \\
\hline Yambot et al. unpubl. & Barcoding & Liza sp. \\
\hline Yambot et al. unpubl. & Barcoding & Chelon macrolepis \\
\hline Ashiq Ur Rahman et al. 2014 & 4 Phylogeny & Moolgarda perusii \\
\hline Ashiq Ur Rahman et al. 2014 & 4 Phylogeny & Moolgarda perusii \\
\hline Ashiq Ur Rahman et al. 2014 & 4 Phylogeny & Moolgarda perusii \\
\hline Ashiq Ur Rahman et al. 2014 & 4 Phylogeny & Moolgarda perusii \\
\hline Ashiq Ur Rahman et al. 2014 & 4 Phylogeny & Moolgarda perusii \\
\hline Ashiq Ur Rahman et al. 2014 & 4 Phylogeny & Moolgarda perusii \\
\hline Ashiq Ur Rahman et al. 2014 & 4 Phylogeny & Moolgarda perusii \\
\hline Ashiq Ur Rahman et al. 2014 & 4 Phylogeny & Moolgarda perusii \\
\hline Ashiq Ur Rahman et al. 2014 & Phylogeny & Moolgarda perusii \\
\hline Khedkar et al. 2014 & Barcoding & Rhinomugil corsula \\
\hline Steinke et al. unpubl. & Barcoding & Liza melinoptera \\
\hline Chen et al. 2015 & Barcoding & Liza haematocheila \\
\hline Juguilon et al. unpubl. & Barcoding & Moolgarda perusii \\
\hline Juguilon et al. unpubl. & Barcoding & Moolgarda seheli \\
\hline Bentley and Wiley unpubl. & - & Crenimugil crenilabis \\
\hline Bentley and Wiley unpubl. & - & Chelon macrolepis \\
\hline Bentley and Wiley unpubl. & - & Liza richardsonii \\
\hline Bentley and Wiley unpubl. & - & Mugil cephalus \\
\hline Bentley and Wiley unpubl. & - & Mugil curema \\
\hline Bentley and Wiley unpubl. & - & Mugil trichodon \\
\hline Bentley and Wiley unpubl. & - & Myxus capensis \\
\hline Bentley and Wiley unpubl. & - & Valamugil buchanan \\
\hline Marucot et al. unpublished & Barcoding & Chelon subviridis \\
\hline Zuccon and Noren unpubl. & Barcoding & Chelon labrosus \\
\hline Zuccon and Noren unpubl. & Barcoding & Chelon labrosus \\
\hline Ordonio et al. unpubl. & Barcoding & Liza macrolepis \\
\hline Ordonio et al. unpubl. & Barcoding & Liza macrolepis \\
\hline Ordonio et al. unpubl. & Barcoding & Liza melinoptera \\
\hline Ordonio et al. unpubl. & Barcoding & Mugil cephalus \\
\hline Ordonio et al. unpubl. & Barcoding & Mugil cephalus \\
\hline Geiger et al. 2014 & Barcoding & Mugil cephalus \\
\hline Geiger et al. 2014 & Barcoding & Liza abu \\
\hline Geiger et al. 2014 & Barcoding & Liza abu \\
\hline Geiger et al. 2014 & Barcoding & Liza ramado \\
\hline Geiger et al. 2014 & Barcoding & Liza aurata \\
\hline & Barcoding & Liza ramado \\
\hline Geiger et al. 2014 & Barcoding & Chelon labros \\
\hline Geiger et al. 2014 & Barcoding & Chelon labrosus \\
\hline
\end{tabular}

Planiliza macrolepis

Planiliza macrolepis

Mugil sp.

Mugil sp.

Mugil sp.

Mugil sp.

Mugil sp.

Mugil sp.

Muil sp.

Mugil sp. I

Mugil sp. I

Mugil sp. I

Mugil sp.

Mugil sp.

Mugil sp.

Mugil sp.I

Mugil sp.I

Mugil sp.I

Mugil sp. I

Mugil sp.I

Mugil sp. I

Planiliza sp.

Osteoniliza sp. $\mathrm{H}$

Osteomugil perusii

Osteomugil perusii

Osteomugil perusii

Osteomugil perusii

Osteomugil perusii

Osteomugil perusi

Osteomugil perusi

Osteomugil perusii

Osteomugil perusi
Rhinomugil corsula

Planiliza melinoptera

Planiliza haematoche

Osteomugil perusii

Crenimugil sp. A

Crenimugil crenilabis
Planiliza macrolepis

Planiliza macrolepis

Mugil sp. B

Mugil sp. R

Pseudomyxus capensis

Crenimugil sp. A

Planiliza subviridis

Chelon labrosus
Chelon labrosus

Planiliza sp. H

Planiliza sp. H
Planiliza sp. H

Planiliza sp. $\mathrm{H}$
Planiliza sp.

Planiliza sp.

Mugil sp. I

Mugil cephalus

Planiliza abu

Planiliza abu

Chelon ramado

Chelon auratus

Chelon ramado

Chelon labrosus
Chelon labrosus 


\begin{tabular}{|c|c|c|c|c|c|c|c|c|c|c|}
\hline KJ552859 & Ex06A6 & ZFMK:50561 & 38.395 N 21.435 E, Mediterranean drainage, Greece & - & - & Geiger et al. 2014 & Barcoding & Liza aurata & Chelon auratus & M \\
\hline KJ552871 & Ex22A8 & ZFMK:FSJF:1892 & 33.889 N 6.999 W, Iqem drainage, Morocco & - & - & Geiger et al. 2014 & Barcoding & Chelon labrosus & Chelon labrosus & M \\
\hline KJ552886 & EX06A3 & ZFMK:50558 & 38.395 N 21.435 E, Mediterranean drainage, Greece & - & - & Geiger et al. 2014 & Barcoding & Liza aurata & Chelon auratus & M \\
\hline KJ552892 & Ex60divC12 & ZFMK:FSJF:LC3 & $33.495 \mathrm{~N} 35.334 \mathrm{E}$, Mediterranean drainage, Lebanon & - & - & Geiger et al. 2014 & Barcoding & Chelon sp. & Planiliza sp. A & u \\
\hline KJ552920 & Ex51A9 & IUSHM 2012-1059 & 41.03 N $28.959 \mathrm{E}$, Istanbul drainage, Turkey & - & - & Geiger et al. 2014 & Barcoding & Liza aurata & Chelon auratus & M \\
\hline KJ552931 & Ex06B3 & ZFMK:50570 & 38.395 N 21.435 E, Mediterranean drainage, Greece & - & - & Geiger et al. 2014 & Barcoding & Chelon labrosus & Chelon labrosus & M \\
\hline KJ552955 & Ex51B10 & IUSHM 2012-1058 & $41.03 \mathrm{~N} 28.959 \mathrm{E}$, Istanbul drainage, Turkey & - & - & Geiger et al. 2014 & Barcoding & Liza ramado & Chelon ramado & M \\
\hline KJ552973 & Ex06A10 & ZFMK:50565 & 38.395 N $21.435 \mathrm{E}$, Mediterranean drainage, Greece & - & - & Geiger et al. 2014 & Barcoding & Liza saliens & Chelon saliens & M \\
\hline KJ553008 & Ex06A7 & ZFMK:50562 & $38.395 \mathrm{~N} 21.435 \mathrm{E}$, Mediterranean drainage, Greece & - & - & Geiger et al. 2014 & Barcoding & Liza aurata & Chelon auratus & M \\
\hline KJ553023 & Ex51D7 & IUSHM 2012-1044 & $41.03 \mathrm{~N} 28.959 \mathrm{E}$, Istanbul drainage, Turkey & - & - & Geiger et al. 2014 & Barcoding & Liza aurata & Chelon auratus & M \\
\hline KJ553053 & Ex06A12 & ZFMK:50567 & $38.395 \mathrm{~N} 21.435 \mathrm{E}$, Mediterranean drainage, Greece & - & - & Geiger et al. 2014 & Barcoding & Chelon labrosus & Chelon labrosus & M \\
\hline KJ553056 & Ex06A8 & ZFMK:50563 & 38.395 N $21.435 \mathrm{E}$, Mediterranean drainage, Greece & - & - & Geiger et al. 2014 & Barcoding & Liza aurata & Chelon auratus & M \\
\hline KJ553076 & Ex06A9 & ZFMK:50564 & $38.395 \mathrm{~N} 21.435 \mathrm{E}$, Mediterranean drainage, Greece & - & - & Geiger et al. 2014 & Barcoding & Liza aurata & Chelon auratus & M \\
\hline KJ553091 & Ex51D3 & IUSHM 2012-1060 & 36.047 N 35.981 E, Orontes drainage, Turkey & - & - & Geiger et al. 2014 & Barcoding & Liza abu & Planiliza abu & M \\
\hline KJ553106 & Ex51A8 & IUSHM 2012-1059 & 41.03 N $28.959 \mathrm{E}$, Istanbul drainage, Turkey & - & - & Geiger et al. 2014 & Barcoding & Liza aurata & Chelon auratus & M \\
\hline KJ553122 & Ex22D6 & ZFMK:FSJF:1931 & $33.889 \mathrm{~N} 6.999 \mathrm{~W}$, lqem drainage, Morocco & - & - & Geiger et al. 2014 & Barcoding & Liza ramado & Chelon ramado & M \\
\hline KJ553132 & Ex06B1 & ZFMK:50568 & 38.395 N 21.435 E, Mediterranean drainage, Greece & - & - & Geiger et al. 2014 & Barcoding & Chelon labrosus & Chelon labrosus & M \\
\hline KJ553145 & Ex37E4 & ZFMK:50575 & 38.395 N $21.435 \mathrm{E}$, Mediterranean drainage, Greece & - & - & Geiger et al. 2014 & Barcoding & Chelon labrosus & Chelon labrosus & M \\
\hline KJ553167 & Ex51C7 & IUSHM 2012-1057 & 41.03 N 28.959 E, Istanbul drainage, Turkey & - & - & Geiger et al. 2014 & Barcoding & Liza saliens & Chelon saliens & M \\
\hline KJ553183 & Ex06A11 & ZFMK:50566 & $38.395 \mathrm{~N} 21.435 \mathrm{E}$, Mediterranean drainage, Greece & - & - & Geiger et al. 2014 & Barcoding & Chelon labrosus & Chelon labrosus & M \\
\hline KJ553192 & Ex22A9 & ZFMK:FSJF:1892 & 33.889 N 6.999 W, Iqem drainage, Morocco & - & . & Geiger et al. 2014 & Barcoding & Chelon labrosus & Chelon labrosus & M \\
\hline KJ553197 & Ex06A1 & ZFMK:50556 & 38.395 N 21.435 E, Mediterranean drainage, Greece & - & - & Geiger et al. 2014 & Barcoding & Liza saliens & Chelon saliens & M \\
\hline KJ553231 & Ex22D10 & ZFMK:FSJF:1935 & 33.889 N 6.999 W, Iqem drainage, Morocco & - & - & Geiger et al. 2014 & Barcoding & Liza ramado & Chelon ramado & M \\
\hline KJ553246 & Ex51C12 & IUSHM 2012-1060 & 36.047 N 35.981 E, Orontes drainage, Turkey & - & - & Geiger et al. 2014 & Barcoding & Liza abu & Planiliza abu & M \\
\hline KJ553734 & Ex19E5 & ZFMK:FSJF:1540 & 37.528 N 31.269 E, Koeprue drainage, Turkey & - & - & Geiger et al. 2014 & Barcoding & Mugil cephalus & Mugil cephalus & M \\
\hline KJ553812 & Ex06B4 & ZFMK:50571 & 38.395 N $21.435 \mathrm{E}$, Mediterranean drainage, Greece & - & . & Geiger et al. 2014 & Barcoding & Mugil cephalus & Mugil cephalus & M \\
\hline KJ553862 & Ex22E4 & ZFMK:FSJF:1939 & 31.467 N 9.759 W, Ksob drainage, Morocco & - & - & Geiger et al. 2014 & Barcoding & Mugil cephalus & Mugil cephalus & M \\
\hline KJ553892 & Ex22E8 & ZFMK:FSJF:1943 & 33.889 N 6.999 W, Iqem drainage, Morocco & - & - & Geiger et al. 2014 & Barcoding & Mugil cephalus & Mugil cephalus & M \\
\hline KJ553924 & Ex06B5 & ZFMK:50572 & 38.395 N 21.435 E, Mediterranean drainage, Greece & - & - & Geiger et al. 2014 & Barcoding & Mugil cephalus & Mugil cephalus & M \\
\hline KJ553935 & Ex19E4 & ZFMK:FSJF:1540 & 37.528 N 31.269 E, Koeprue drainage, Turkey & - & - & Geiger et al. 2014 & Barcoding & Mugil cephalus & Mugil cephalus & M \\
\hline KJ622047 & & & & . & . & W. Chu, unpubl. & & Liza haematocheila & Planiliza haematocheila & M \\
\hline
\end{tabular}

a EF609279. EF609494 not retained for barcode identification because used as reference bv Durand et al. (2012b) JF952795. JF952796 not retained because of likelv confusion in the labelina of samples

JO842245 not retained because lenath of seauence insufficient 
Supplementary Fig. S1 Phylogenetic trees of mitochondrial haplotypes constructed from partitioned maximum-likelihood analysis of 3,885 aligned nucleotides from 16S rRNA, COI and cytb gene sequences (edited from Durand et al. 2012b). Bootstrap scores $>50 \%$ are indicated. Asterisks indicate nodes with a posteriori probability from partitioned Bayesian analysis $>0.95$. Vertical bars on the right of the tree indicate statistically supported lineages that have been considered to represent distinct species (Durand and Borsa 2015) a Mugil cephalus species complex. b Mugil curema species complex

Supplementary Fig. S2 Phylogenetic trees of mitochondrial haplotypes constructed from partitioned maximum-likelihood analysis of 3,885 aligned nucleotides from 16S rRNA, COI and cytb gene sequences (edited from Durand et al. 2012b). Bootstrap scores $>50 \%$ are indicated. Asterisks indicate nodes with a posteriori probability from partitioned Bayesian analysis $>0.95$. Vertical bars on the right of the tree indicate statistically supported lineages that have been considered to represent distinct species (Durand and Borsa 2015). a Genera Osteomugil and Crenimugil, as redefined by Durand et al. (2012a). b Genera Chelon and Planiliza, as redefined by Durand et al. (2012a)

Supplementary Fig. S3 Neighbor-joining tree of 829 partial COI-gene sequences belonging to Mugilidae, including the 261 sequences of Durand et al. (2012 a,b) used as reference and 568 other sequences retrieved from GenBank on 21 February 2015. Distances were estimated using the K2P model in MEGA5 (Tamura et al. 2011). Bootstrap score indicated at a node when $>70 \%$. Vertical bars on the right of the tree indicate strongly-supported lineages that represent distinct species according to Durand and Borsa (2015). Sub-trees of the Mugil cephalus and the M. curema species complexes are shown in Supplementary Figs. 4 and 5, respectively; those for the genera Osteomugil, Crenimugil, Chelon, and Planiliza are shown in Supplementary Figs. 6-9, respectively

Supplementary Fig. S4 Detail of sub-tree that represents COI haplotypes in the Mugil cephalus species complex, from the NJ tree of Supplementary Fig. S3. Bootstrap score indicated at a node when $>70 \%$. Vertical bars on the right of the tree indicate strongly-supported lineages that represent distinct species according to Durand and Borsa (2015)

Supplementary Fig. S5 Detail of sub-tree that represents COI haplotypes in the Mugil curema species complex, from the NJ tree of Supplementary Fig. S3. Bootstrap score indicated at a node when $>70 \%$. Vertical bars on the right of the tree indicate strongly-supported lineages that represent distinct species according to Durand and Borsa (2015)

Supplementary Fig. S6 Detail of sub-tree that represents COI haplotypes in the genus Osteomugil, from the NJ tree of Supplementary Fig. S3. Bootstrap score indicated at a node when $>70 \%$. Vertical bars on the right of the tree indicate strongly-supported lineages that represent distinct species according to Durand and Borsa (2015)

Supplementary Fig. S7 Detail of sub-tree that represents COI haplotypes in the genus Crenimugil, from the NJ tree of Supplementary Fig. S3. Bootstrap score indicated at a node when $>70 \%$. Vertical bars on the right of the tree indicate strongly-supported lineages that represent distinct species according to Durand and Borsa (2015)

Supplementary Fig. S8 Detail of sub-tree that represents COI haplotypes in the genus Chelon, from the NJ tree of Supplementary Fig. S3. Bootstrap scores are indicated at a node when $>70 \%$. Vertical bars on the right of the tree indicate strongly-supported lineages that represent distinct species according to Durand and Borsa (2015)

Supplementary Fig. S9 Detail of sub-tree that represents COI haplotypes in the genus Planiliza, from the NJ tree of Supplementary Fig. S3. Bootstrap scores indicated at a node when $>70 \%$. Vertical bars on the right of the tree indicate strongly-supported lineages that represent distinct species according to Durand and Borsa (2015) 

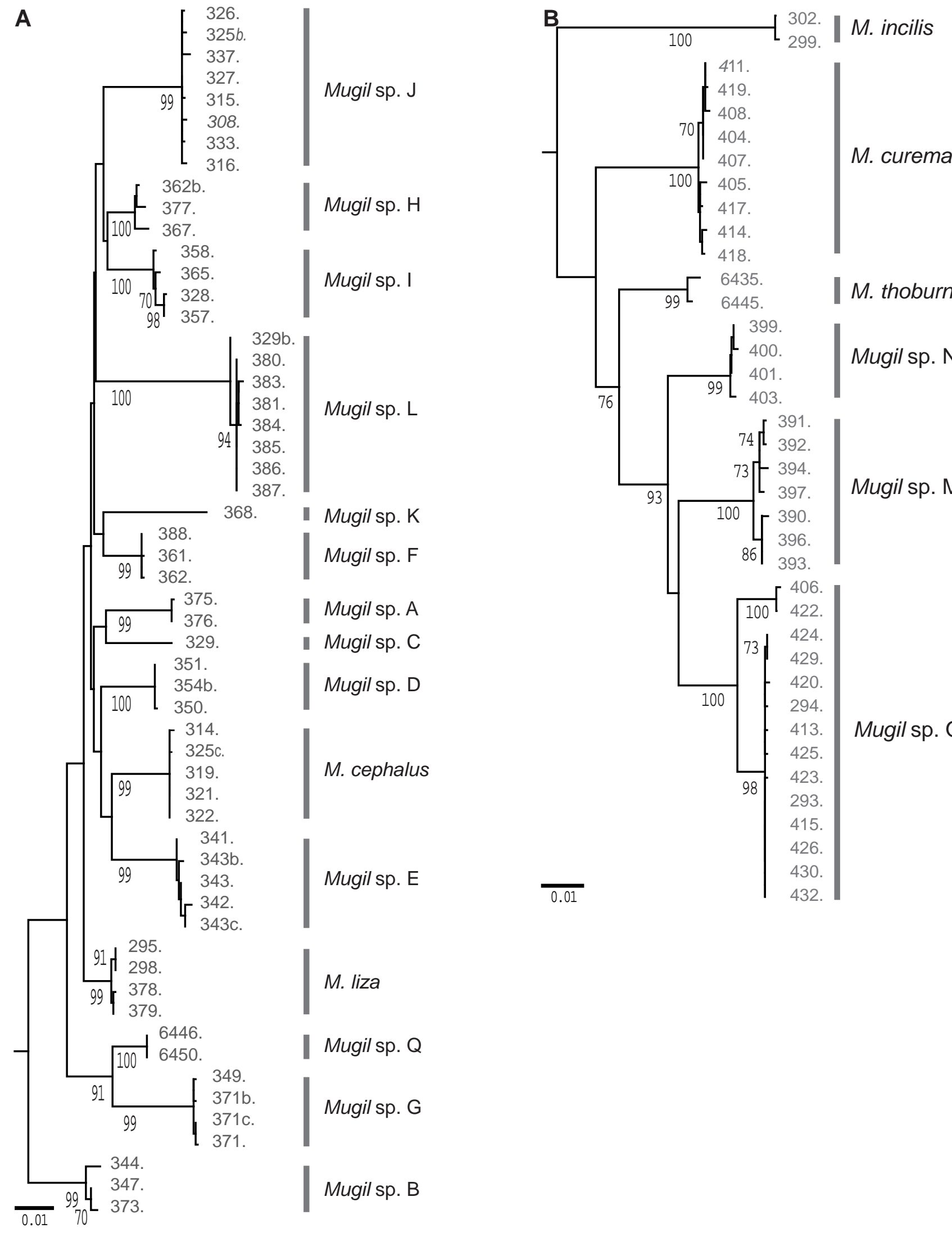

M. curema

M. thoburni Mugil sp. N

Mugil sp. M $30-394$

93 397. 396. 86393.

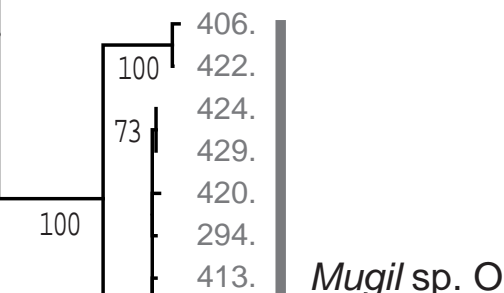

91. 295.

$99+378$.

M. liza

Mugil sp. Q

Mugil sp. G

Mugil sp. B 

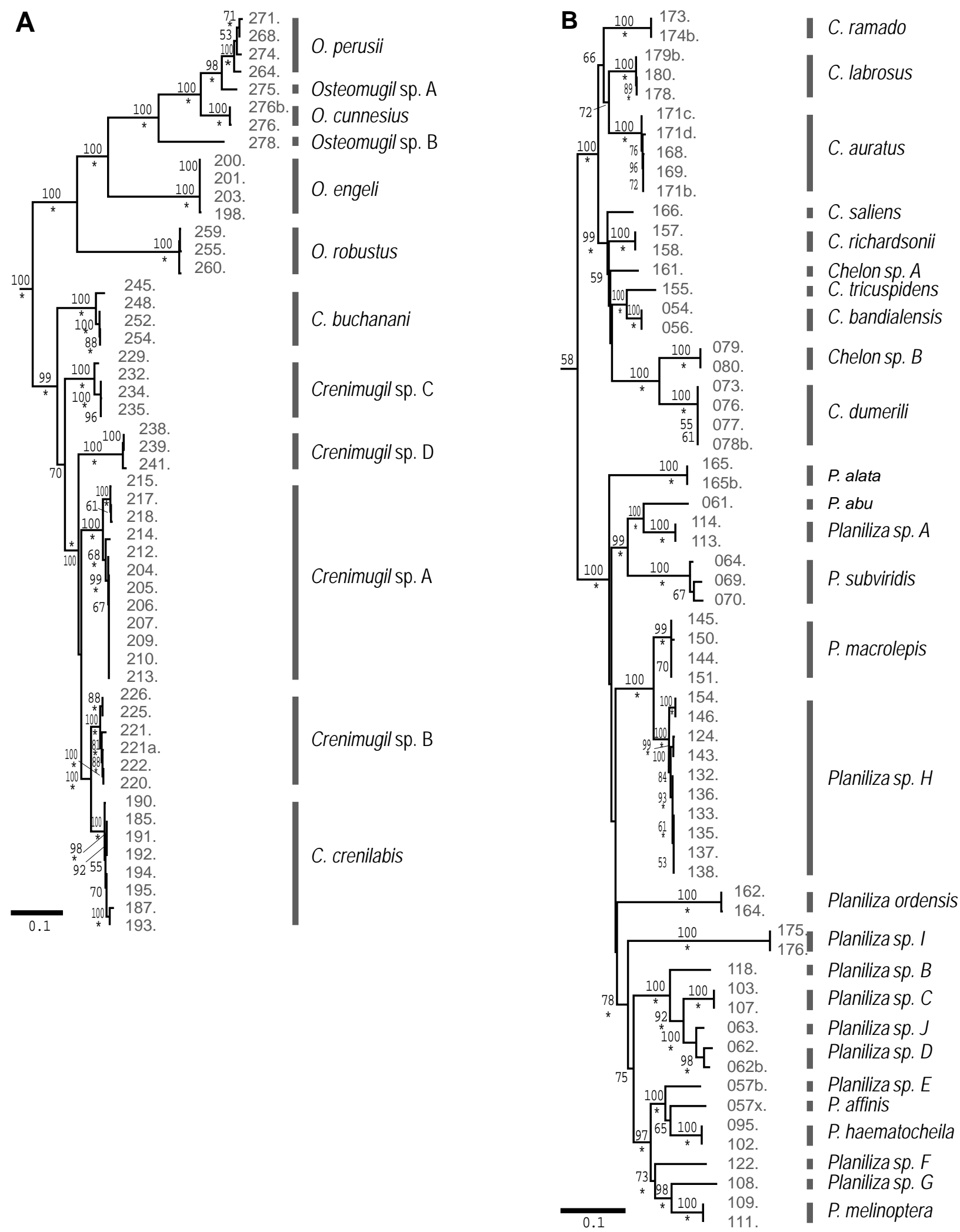


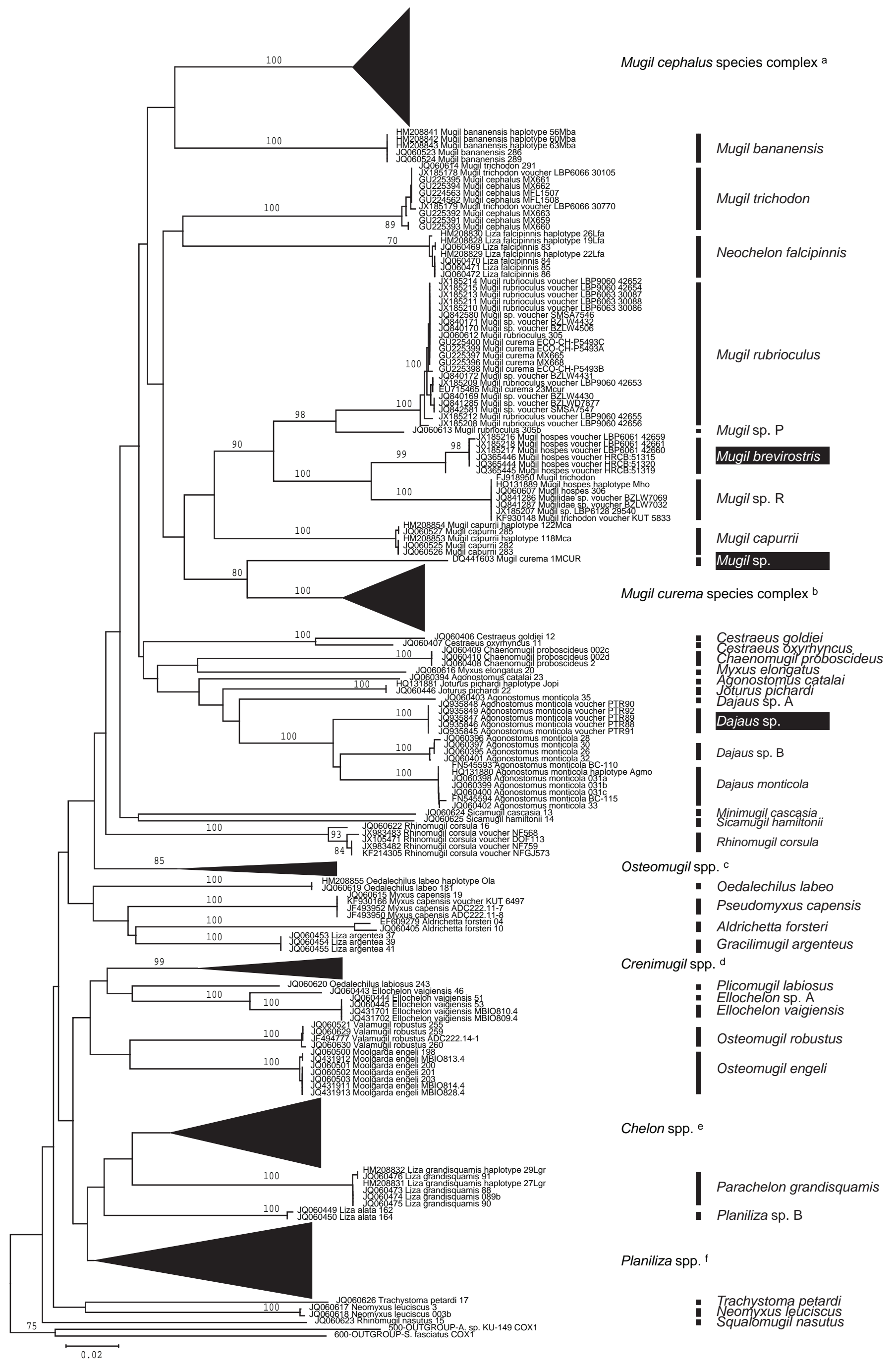




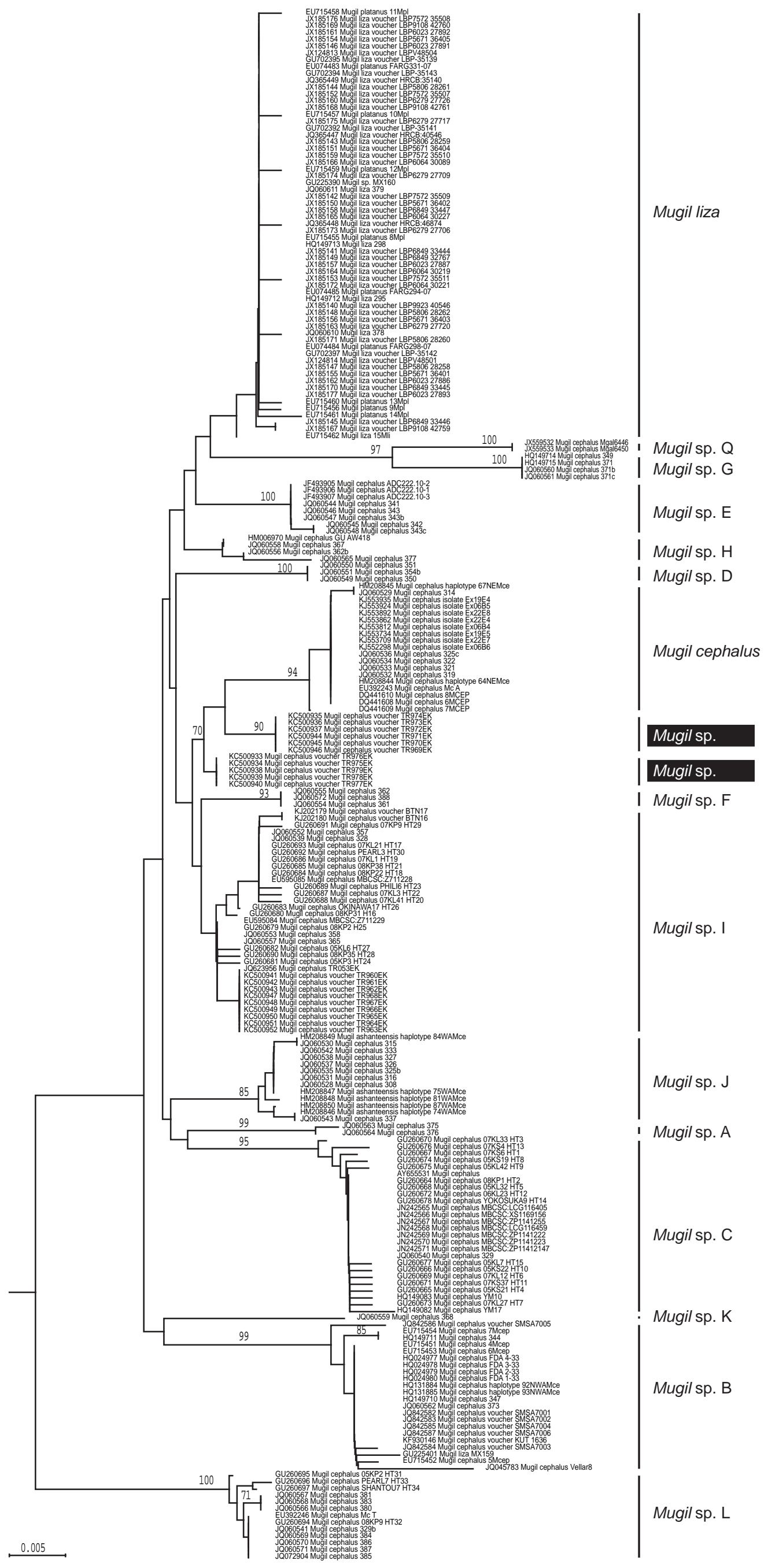

Supplementary Fig. S4 


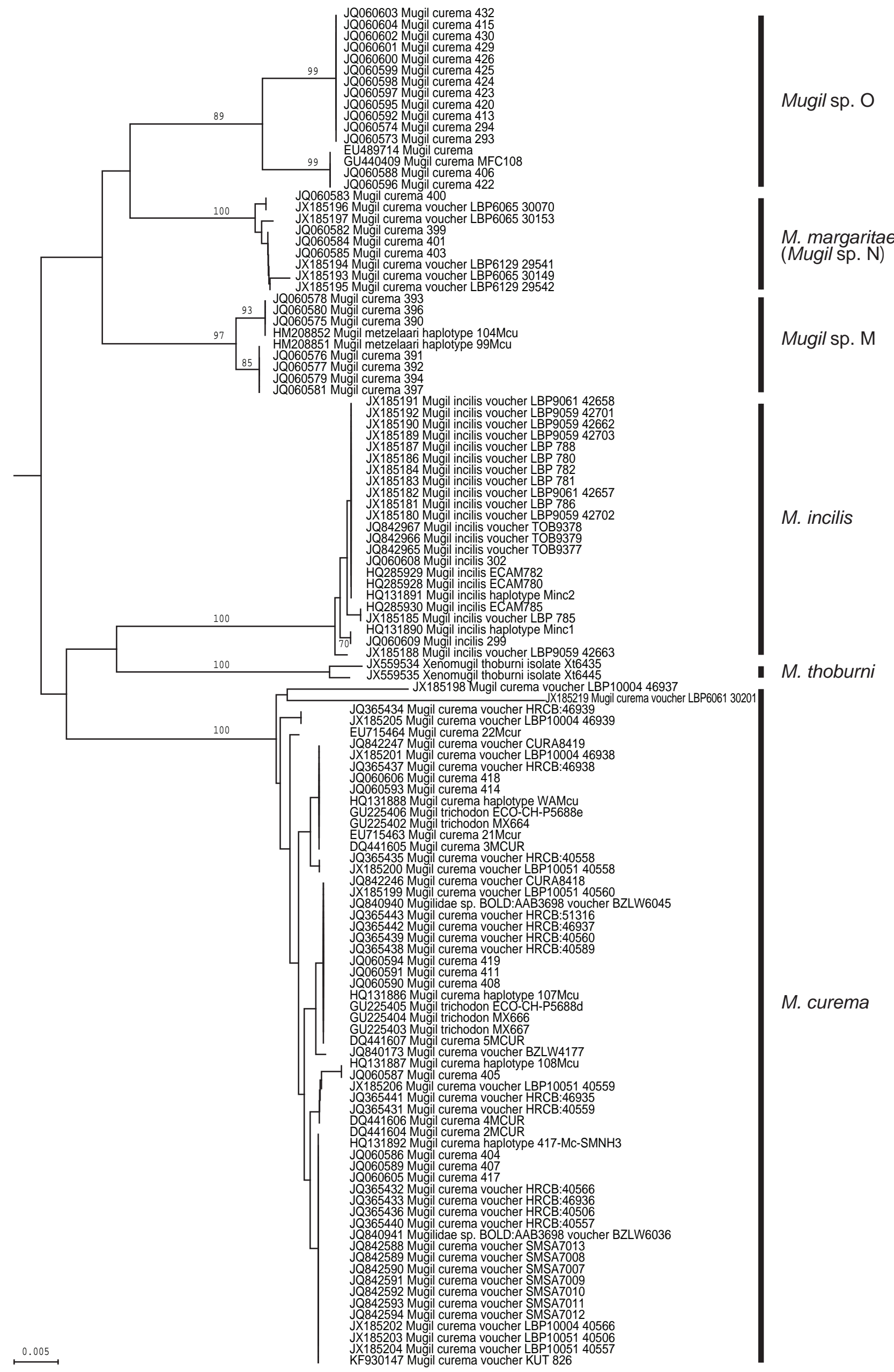




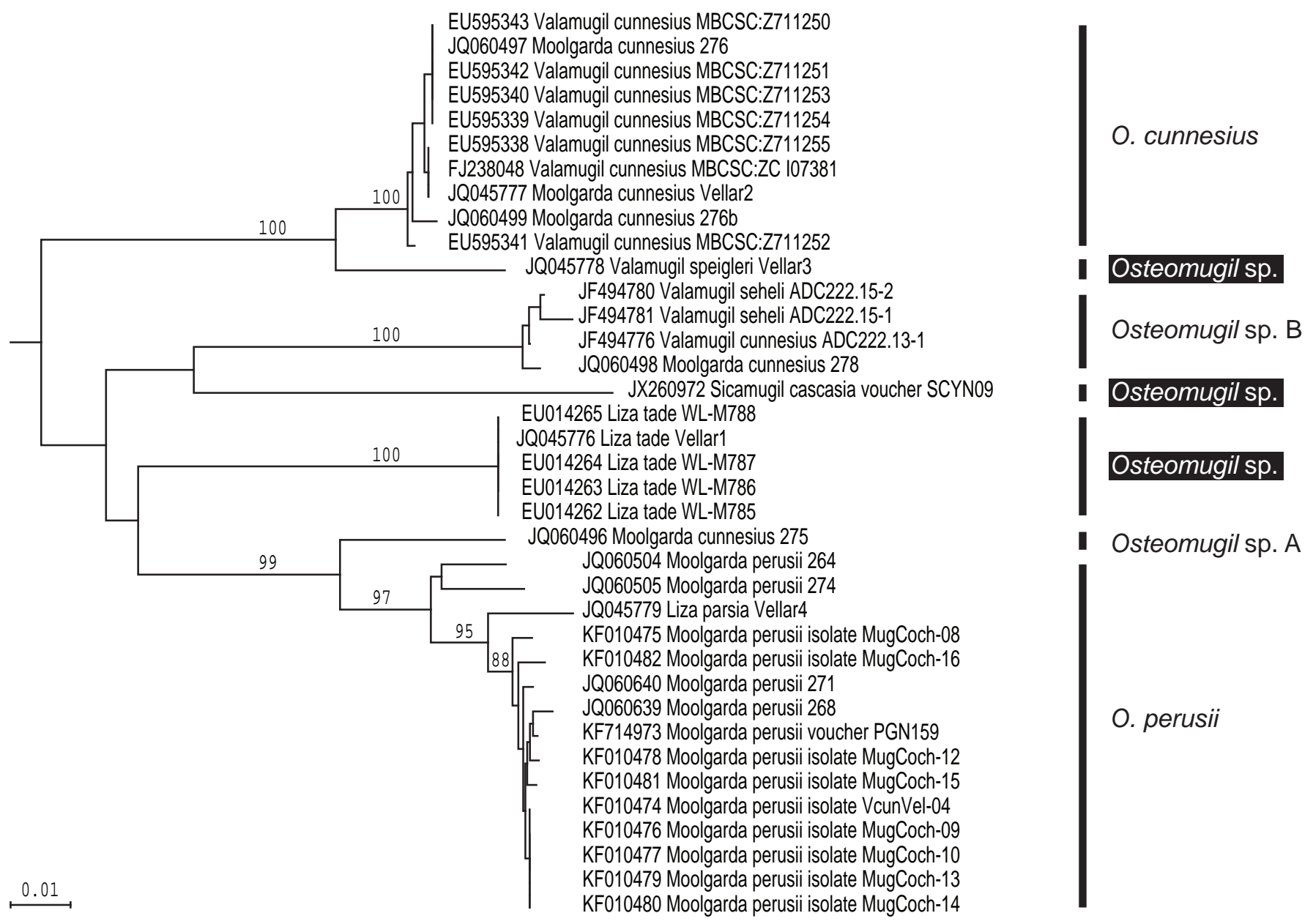

\section{Supplementary Fig. S6}




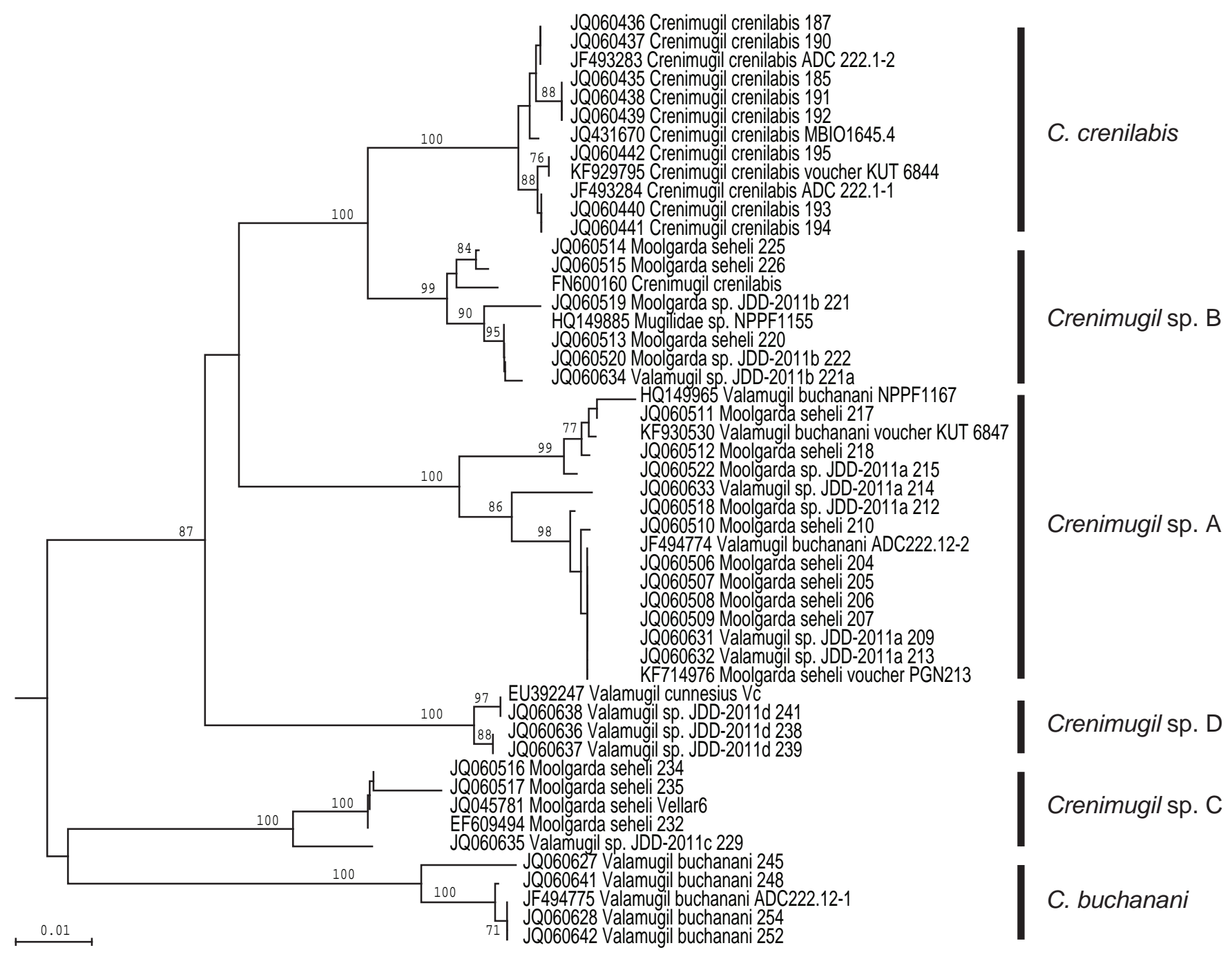

Supplementary Fig. S7 


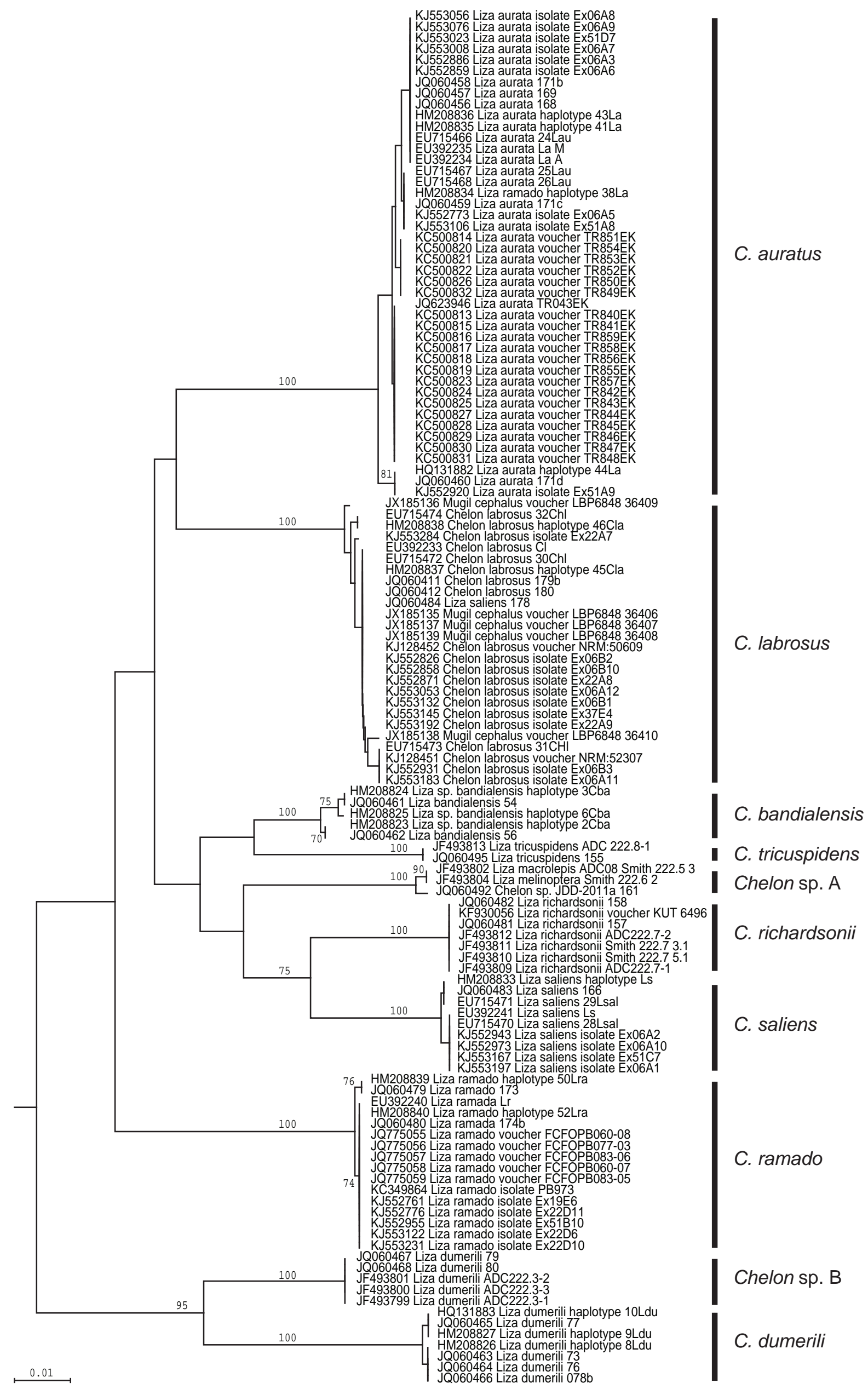




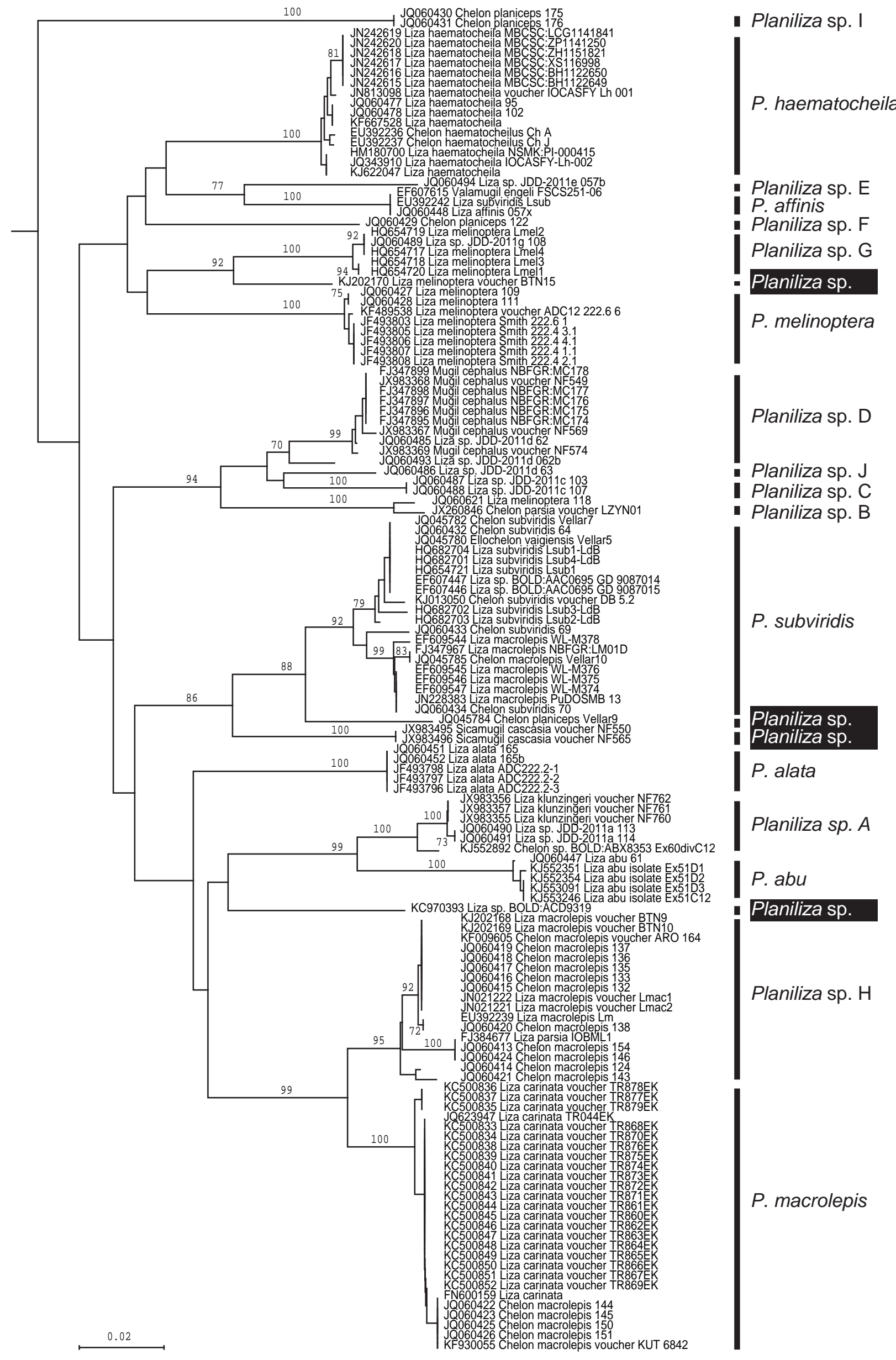

Supplementary Fig. S9 